\title{
Direct hydrofluorination of methallyl alkenes using a methanesulfonic acid/triethylamine trihydrofluoride combination
}

\author{
Xavier Bertrand and Jean-François Paquin* \\ CGCC, PROTEO, Département de chimie, Université Laval, 1045 avenue de la Médecine, Québec, \\ Québec, G1V 0A6, Canada \\ *Corresponding author: jean-francois.paquin@ chm.ulaval.ca
}

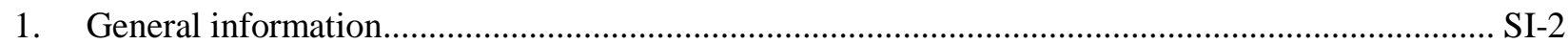

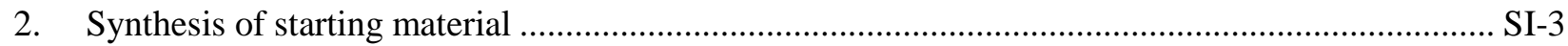

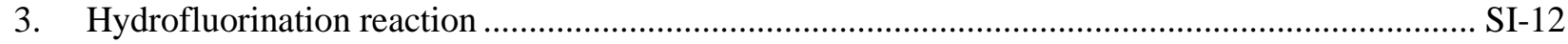

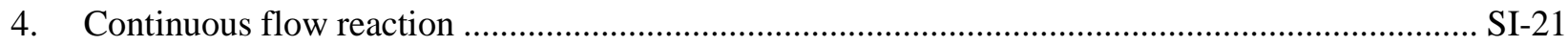

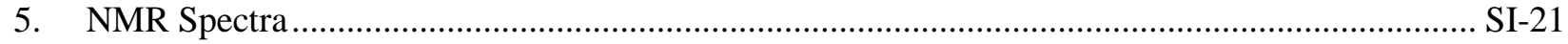




\section{General information}

The following includes general experimental procedures, specific details for representative reactions, isolation and spectroscopic information for the new compounds prepared. All commercial compounds were used as received. Solvents were used as purchased unless stated as dry. THF and $\mathrm{CH}_{2} \mathrm{Cl}_{2}$ were purified using a Vacuum Atmospheres Inc. Solvent Purification System. All air and water sensitive reactions were carried out under argon atmosphere. Reactions were monitored by TLC on pre-coated plates (Silicycle silica gel 60 Å F254 230-240 mesh) and products were visualized under $254 \mathrm{~nm}$ UV light followed by staining with $\mathrm{KMnO}_{4}$ when appropriate. Purification by flash column chromatography was carried out on silica gel (Silicycle silica gel $60 \AA$ A F254). Continuous flow reactions were performed on a Vapourtec E-Series. NMR spectra were recorded on an Agilent DD2 $500 \mathrm{MHz}$ spectrometer or on a Varian Inova $400 \mathrm{MHz}$ spectrometer in the indicated solvent at $298 \mathrm{~K}$. Chemical shifts for ${ }^{1} \mathrm{H}$ and ${ }^{13} \mathrm{C}$ spectra are reported on the delta scale in ppm and were referenced to residual solvent references or internal TMS reference. Resonances are reported as follows: chemical shift $(\delta \mathrm{ppm})$, multiplicity $(\mathrm{s}=$ singulet, $\mathrm{d}$ $=$ doublet, $\mathrm{t}=$ triplet, $\mathrm{q}=$ quartet, $\mathrm{p}=$ quintet, $\mathrm{m}=$ multiplet, br. $\mathrm{s}=$ broad signal or a combination of the above), coupling constant $(\mathrm{Hz})$, integration. NMR yields were determined by ${ }^{19} \mathrm{~F}$ NMR analysis of the crude mixture after work-up using 2-fluoro-4-nitrotoluene as the internal standard. High-resolution mass (HRMS) spectra were obtained on a LC/MS-TOF Agilent 6210 using electrospray ionization (ESI) or atmospheric pressure photoionization (APPI). Infrared spectra were recorded on an ABB MB3000 FT-IR spectrometer. Optical rotations were measured on a Jasco DIP-360 Polarimeter with a sodium lamp at ambient temperature. 


\section{Synthesis of starting material}

General procedure A: $N$-Ethyl- $N$-(3-dimethylaminopropyl)carbodiimide hydrochloride (EDC· $\mathrm{HCl}, 1.3$ equiv.), $N$-methylmorpholine (1.3 equiv.) and $N, N$-dimethylaminopyridine (DMAP, 0.1 equiv.) were added to $\mathrm{CH}_{2} \mathrm{Cl}_{2}(0.25 \mathrm{M})$ at $0{ }^{\circ} \mathrm{C}$, followed by the corresponding carboxylic acid (1 equiv.). 3-methyl-3buten-1-ol (1.2 equiv.) was added, and the reaction was warmed to room temperature and stirred overnight. The organic layer was then washed twice with aq. $\mathrm{HCl}(10 \%)$, once with sat. aq. $\mathrm{NaHCO}_{3}$ and once with brine. The organic layer was dried over $\mathrm{MgSO}_{4}$, filtered and concentrated under reduced pressure. Purification by flash chromatography afforded the final product.

General procedure B: The corresponding acid (1.1 equiv.) and 1,1'-carbonyldiimidazole (CDI, 1.1 equiv.) were added to $\mathrm{CH}_{2} \mathrm{Cl}_{2}(0.2 \mathrm{M})$ and the mixture was stirred at room temperature for $1 \mathrm{~h}$. 3-methyl3-buten-1-ol (1 equiv.) was then added to the solution and the mixture was stirred at room temperature overnight. The mixture was washed with aq. sat. $\mathrm{NaHCO}_{3}$ and brine, dried over $\mathrm{Na}_{2} \mathrm{SO}_{4}$, filtered and concentrated under reduced pressure. Purification by flash chromatography afforded the final product.

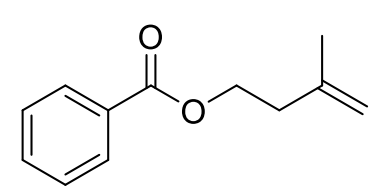

\section{3-methylbut-3-en-1-yl benzoate (1a).}

3-methyl-3-buten-1-ol (1.0 mL, $10 \mathrm{mmol}, 1$ equiv.) and $\mathrm{Et}_{3} \mathrm{~N}$ (2.8 mL, $20 \mathrm{mmol}$, 2.1 equiv.) were added to $\mathrm{CH}_{2} \mathrm{Cl}_{2}(17 \mathrm{~mL}, 0.6 \mathrm{M})$. The mixture was cooled down to $0{ }^{\circ} \mathrm{C}$ and benzoyl chloride $(1.2 \mathrm{~mL}, 10 \mathrm{mmol}, 1$ equiv.) was added. The mixture was stirred overnight at room temperature. $10 \%$ aq. $\mathrm{HCl}$ was then added, and the organic layer was washed with $10 \%$ aq. $\mathrm{HCl}$ (2x), sat. aq. $\mathrm{NaHCO}_{3}$ and brine. The organic layer was then dried with $\mathrm{Na}_{2} \mathrm{SO}_{4}$ and concentrated under reduced pressure. Purification by flash chromatography (10\% EtOAc/hexanes) afforded the desired product $(1.72 \mathrm{~g}, 90 \%)$ as a colorless oil. Spectroscopic data agreed with the literature. ${ }^{1}{ }^{1} \mathrm{H}$ NMR (500 $\left.\mathrm{MHz}, \mathrm{CDCl}_{3}\right) \delta 8.06-8.01(\mathrm{~m}, 2 \mathrm{H}), 7.58-7.53(\mathrm{~m}, 1 \mathrm{H}), 7.46-7.41(\mathrm{~m}, 2 \mathrm{H}), 4.86-4.84(\mathrm{~m}, 1 \mathrm{H}), 4.83$ $-4.81(\mathrm{~m}, 1 \mathrm{H}), 4.44(\mathrm{t}, J=6.8 \mathrm{~Hz}, 2 \mathrm{H}), 2.49(\mathrm{t}, J=6.8 \mathrm{~Hz}, 2 \mathrm{H}), 1.82(\mathrm{dd}, J=1.5,0.9 \mathrm{~Hz}, 3 \mathrm{H})$.

\footnotetext{
${ }^{1}$ Xie, Y.; Sun, P.-W.; Li, Y.; Wang, S.; Ye, M.; Li, Z. Angew. Chem. Int. Ed. 2019, 58, 7097-7101
} 
<smiles>C=C(C)CCOC(=O)c1ccc(C(C)(C)C)cc1</smiles>

\section{3-methylbut-3-en-1-yl 4-(tert-butyl)benzoate (1b).}

Following the general procedure B on a $1 \mathrm{mmol}$ scale and using 4-(tertbutyl)benzoic acid, the desired product was obtained as a colorless oil (202 $\mathrm{mg}, 0.82 \mathrm{mmol}, 82 \%)$ after purification by flash chromatography (10\% EtOAc/hexanes). Spectroscopic data agreed with the literature. ${ }^{2}{ }^{1} \mathrm{H}$ NMR (400 MHz, $\left.\mathrm{CDCl}_{3}\right) \delta 8.00-7.92$ (m, 2H), $7.49-7.41(\mathrm{~m}, 2 \mathrm{H}), 4.85-4.82(\mathrm{~m}, 1 \mathrm{H}), 4.82-4.80(\mathrm{~m}, 1 \mathrm{H}), 2.47$ (t, $J=6.7 \mathrm{~Hz}, 2 \mathrm{H}), 1.81$ (s, $3 \mathrm{H}), 1.34(\mathrm{~s}, 9 \mathrm{H})$.

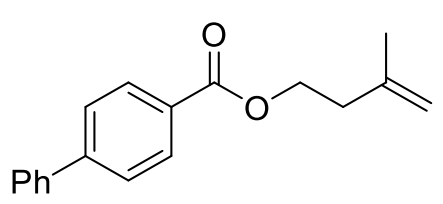

\section{3-methylbut-3-en-1-yl [1,1'-biphenyl]-4-carboxylate (1c)}

Following the general procedure $\mathrm{B}$ on a 1 mmol scale using 4biphenylcarboxylic acid, the desired product was obtained as a colorless oil (187 mg, $0.7 \mathrm{mmol}, 70 \%$ ) after purification by flash chromatography (10\% EtOAc/hexanes). Spectroscopic data agreed with the literature. ${ }^{11} \mathrm{H}$ NMR $\left(400 \mathrm{MHz}, \mathrm{CDCl}_{3}\right) \delta 8.14-8.06(\mathrm{~m}, 2 \mathrm{H}), 7.70-$ 7.60 (m, 4H), $7.52-7.44(\mathrm{~m}, 2 \mathrm{H}), 7.39$ (m, 1H), $4.86(\mathrm{~s}, 1 \mathrm{H}), 4.83$ (s, 1H), 4.46 (t, $J=6.8 \mathrm{~Hz}, 2 \mathrm{H}), 2.51$ $(\mathrm{t}, J=6.8 \mathrm{~Hz}, 2 \mathrm{H}), 1.83(\mathrm{~s}, 3 \mathrm{H})$.

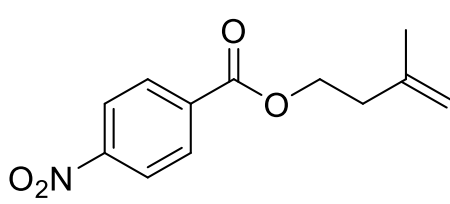

\section{3-methylbut-3-en-1-yl 4-nitrobenzoate (1d)}

Following the general procedure A on a $0.6 \mathrm{mmol}$ scale using 4-nitrobenzoic acid, the desired product was obtained as a yellowish oil (103 $\mathrm{mg}, 0.44$ $\mathrm{mmol}, 73 \%$ ) after purification by flash chromatography (10\% EtOAc/hexanes). IR (ATR, diamond), $v=$ 3080, 2970, 1720, 1609, 1526, 1352, 1266, 1104, 1014, $874 \mathrm{~cm}^{-1} ;{ }^{1} \mathrm{H}$ NMR $\left(500 \mathrm{MHz}, \mathrm{CDCl}_{3}\right) \delta 8.31-$ $8.26(\mathrm{~m}, 2 \mathrm{H}), 8.23-8.18(2 \mathrm{H}, \mathrm{m}), 4.86(1 \mathrm{H}, \mathrm{s}), 4.82(1 \mathrm{H}, \mathrm{s}), 4.50(2 \mathrm{H}, \mathrm{t}, J=6.7 \mathrm{~Hz}), 2.52(2 \mathrm{H}, \mathrm{t}, J=7.1$ $\mathrm{Hz}), 1.83(3 \mathrm{H}, \mathrm{s}) ;{ }^{13} \mathrm{C} \mathrm{NMR}\left(126 \mathrm{MHz}, \mathrm{CDCl}_{3}\right) \delta 164.7,150.6,141.4,135.8,130.7,123.6,112.8,64.0$, 36.8, 25.5; HRMS-ESI calcd for $\mathrm{C}_{12} \mathrm{H}_{13} \mathrm{NNaO}_{4}[\mathrm{M}+\mathrm{Na}]^{+}$258.0730, found 258.0737.

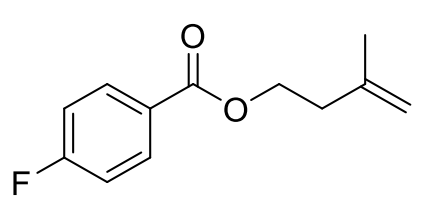

\section{3-methylbut-3-en-1-yl 4-fluorobenzoate (1e)}

Following the general procedure B on a $1 \mathrm{mmol}$ scale using 4-fluorobenzoic acid, the desired product was obtained as a colorless oil $(157 \mathrm{mg}, 0.75 \mathrm{mmol}$, $75 \%)$ after purification by flash chromatography (10\% EtOAc/hexanes). Spectroscopic data agreed with

\footnotetext{
${ }^{2}$ Liu, Y.; Lin, Q.; Xiao, Z.; Zheng, C.-G.; Guo, Y.; Chen, Q.-Y.; Liu, C. Chem. Eur. J. 2019, 25, 1824 - 1828
} 
the literature. ${ }^{11} \mathrm{H} \mathrm{NMR}\left(400 \mathrm{MHz}, \mathrm{CDCl}_{3}\right) \delta 8.05(\mathrm{dd}, J=8.7,5.6 \mathrm{~Hz}, 2 \mathrm{H}), 7.10(\mathrm{t}$ app., $J=8.7 \mathrm{~Hz}, 2 \mathrm{H})$, $4.84(\mathrm{~s}, 1 \mathrm{H}), 4.81(\mathrm{~s}, 1 \mathrm{H}), 4.43(\mathrm{t}, J=6.7 \mathrm{~Hz}, 2 \mathrm{H}), 2.48(\mathrm{t}, J=6.6 \mathrm{~Hz}, 2 \mathrm{H}), 1.81(\mathrm{~s}, 3 \mathrm{H})$.

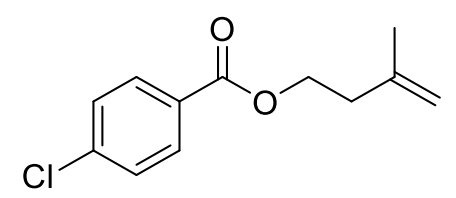

\section{3-methylbut-3-en-1-yl 4-chlorobenzoate (1f)}

Following the general procedure B on a $1 \mathrm{mmol}$ scale using 4-chlorobenzoic acid, the desired product was obtained as a colorless oil $(184 \mathrm{mg}, 0.82 \mathrm{mmol}$, $82 \%)$ after purification by flash chromatography (5\% EtOAc/hexanes). Spectroscopic data agreed with the literature. ${ }^{3}{ }^{1} \mathrm{H}$ NMR $\left(500 \mathrm{MHz}, \mathrm{CDCl}_{3}\right) \delta 7.98-7.95(\mathrm{~m}, 2 \mathrm{H}), 7.43-7.39(\mathrm{~m}, 2 \mathrm{H}), 4.85-4.83(\mathrm{~m}$, $1 \mathrm{H}), 4.81-4.79(\mathrm{~m}, 1 \mathrm{H}), 4.43(\mathrm{t}, J=6.7 \mathrm{~Hz}, 2 \mathrm{H}), 2.48(\mathrm{t}, J=6.7 \mathrm{~Hz}, 2 \mathrm{H}), 1.81(\mathrm{dd}, J=1.5,0.9 \mathrm{~Hz}, 3 \mathrm{H})$.

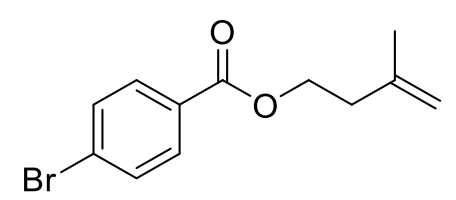

\section{3-methylbut-3-en-1-yl 4-bromobenzoate (1g)}

Following the general procedure $\mathrm{B}$ on a $1 \mathrm{mmol}$ scale using 4-bromobenzoic acid, the desired product was obtained as a colorless oil $(264 \mathrm{mg}, 1 \mathrm{mmol}$, $>99 \%$ ) after purification by flash chromatography (5\% EtOAc/hexanes). Spectroscopic data agreed with the literature. ${ }^{2}{ }^{1} \mathrm{H}$ NMR $\left(500 \mathrm{MHz}, \mathrm{CDCl}_{3}\right) \delta 7.91-7.87(\mathrm{~m}, 2 \mathrm{H}), 7.59-7.55(\mathrm{~m}, 2 \mathrm{H}), 4.86-4.83(\mathrm{~m}$, $1 \mathrm{H}), 4.81-4.79(\mathrm{~m}, 1 \mathrm{H}), 4.43(\mathrm{t}, J=6.8 \mathrm{~Hz}, 2 \mathrm{H}), 2.48(\mathrm{t}, J=6.8 \mathrm{~Hz}, 2 \mathrm{H}), 1.81(\mathrm{dd}, J=1.5,0.9 \mathrm{~Hz}, 3 \mathrm{H})$.

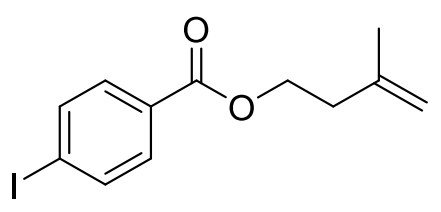

\section{3-methylbut-en-1-yl 4-iodobenzoate (1h)}

Following the general procedure A on a 1 mmol scale using 4-iodobenzoic acid, the desired product was obtained as a colorless oil $(248 \mathrm{mg}, 0.78 \mathrm{mmol}$, $78 \%$ ) after purification by flash chromatography (10\% EtOAc/hexanes). Spectroscopic data agreed with the literature. ${ }^{1}{ }^{1} \mathrm{H} \mathrm{NMR}\left(400 \mathrm{MHz}, \mathrm{CDCl}_{3}\right) \delta 7.84-7.77(\mathrm{~m}, 2 \mathrm{H}), 7.77-$ $7.70(\mathrm{~m}, 2 \mathrm{H}), 4.84(\mathrm{~s}, 1 \mathrm{H}), 4.80(\mathrm{~s}, 1 \mathrm{H}), 4.43(\mathrm{t}, J=6.8 \mathrm{~Hz}, 2 \mathrm{H}), 2.47(\mathrm{t}, J=6.7 \mathrm{~Hz}, 2 \mathrm{H}), 1.80(\mathrm{~s}, 3 \mathrm{H})$.

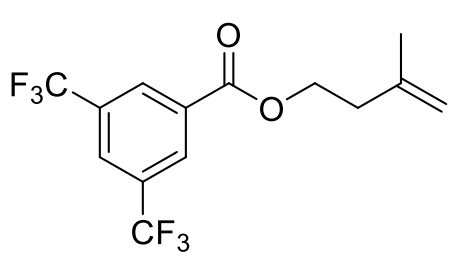

\section{3-methylbut-3-en-1-yl 3,5-bis(trifluoromethyl)benzoate (1i)}

Following the general procedure $\mathrm{B}$ on a 1 mmol scale using 3,5bis(trifluoromethyl)benzoic acid, the desired product was obtained as a colorless oil (203 mg, $0.62 \mathrm{mmol}, 62 \%$ ) after purification by flash

\footnotetext{
${ }^{3}$ Fang, J.; Wang, Z.-K.; Wu, S.-W.; Shen, W.-G.; Ao, G.-Z.; Liu, F. Chem. Commun. 2017, 53, 7638-7641
} 
chromatography (5\% EtOAc/hexanes). IR (ATR, diamond), $v=2972,1732,1624,1371,1277,1244$, 1175, 1130, 912, $770 \mathrm{~cm}^{-1}$; ${ }^{1} \mathrm{H}$ NMR $\left(500 \mathrm{MHz}, \mathrm{CDCl}_{3}\right) \delta 8.48(\mathrm{~s}, 2 \mathrm{H}), 8.06(\mathrm{~s}, 1 \mathrm{H}), 4.89-4.84(\mathrm{~m}, 1 \mathrm{H})$, $4.84-4.79(\mathrm{~m}, 1 \mathrm{H}), 4.53(\mathrm{t}, J=6.8 \mathrm{~Hz}, 2 \mathrm{H}), 2.53(\mathrm{t}, J=6.8 \mathrm{~Hz}, 2 \mathrm{H}), 1.83(\mathrm{~s}, 3 \mathrm{H}) ;{ }^{13} \mathrm{C} \mathrm{NMR}(126 \mathrm{MHz}$, $\left.\mathrm{CDCl}_{3}\right) \delta 163.9,141.2,132.6,132.2(\mathrm{q}, J=33.9 \mathrm{~Hz}), 129.8(\mathrm{q}, J=3.8 \mathrm{~Hz}), 126.4$ (hept, $\left.J=3.7 \mathrm{~Hz}\right), 123.0$ $(\mathrm{q}, J=272.8 \mathrm{~Hz}), 112.9,64.2,36.8,22.5 ;{ }^{19} \mathrm{~F} \mathrm{NMR}\left(470 \mathrm{MHz}, \mathrm{CDCl}_{3}\right) \delta-63.0(\mathrm{~s}, 6 \mathrm{~F})$; HRMS-APPI calcd for $\mathrm{C}_{14} \mathrm{H}_{13} \mathrm{~F}_{6} \mathrm{O}_{2}[\mathrm{M}+\mathrm{H}]^{+}$327.0814, found 327.0826.

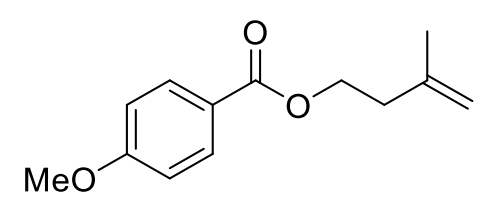

\section{3-methylbut-3-en-1-yl 4-methoxybenzoate (1j)}

Following the general procedure A on a $0.6 \mathrm{mmol}$ scale using 4-anisidic acid, the desired product was obtained as a colorless oil $(97 \mathrm{mg}, 0.44 \mathrm{mmol}$, $73 \%)$ after purification by flash chromatography (10\% EtOAc/hexanes). Spectroscopic data agreed with the literature. ${ }^{1}{ }^{1} \mathrm{H}$ NMR $\left(400 \mathrm{MHz}, \mathrm{CDCl}_{3}\right) \delta$ 8.03-7.95 (m, 2H), 6.95-6.87 (m, 2H), $4.84(\mathrm{~s}, 1 \mathrm{H}), 4.81(\mathrm{~s}$, $1 \mathrm{H}), 4.40(\mathrm{t}, J=6.8 \mathrm{~Hz}, 2 \mathrm{H}), 3.86(\mathrm{~s}, 3 \mathrm{H}), 2.47(\mathrm{t}, J=6.7 \mathrm{~Hz}, 2 \mathrm{H}), 1.81(\mathrm{~s}, 3 \mathrm{H})$.

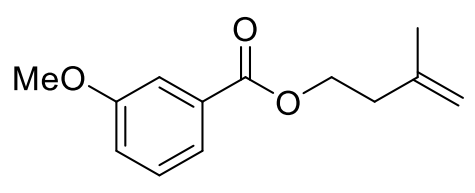

\section{3-methylbut-3-en-1-yl 3-methoxybenzoate (1k)}

Following the general procedure A on a $0.6 \mathrm{mmol}$ scale using 3methoxybenzoic acid, the desired product was obtained as a colorless oil (104 mg, $0.47 \mathrm{mmol}, 79 \%)$ after purification by flash chromatography (10\% EtOAc/hexanes). Spectroscopic data agreed with the literature. ${ }^{1}{ }^{1} \mathrm{H}$ NMR $\left(400 \mathrm{MHz}, \mathrm{CDCl}_{3}\right) \delta 7.63$ (ddd, $J=7.7,1.5,1.1$ $\mathrm{Hz}, 1 \mathrm{H}), 7.56(\mathrm{dd}, J=2.7,1.5 \mathrm{~Hz}, 1 \mathrm{H}), 7.34(\mathrm{~m}, 1 \mathrm{H}), 7.10(\mathrm{ddd}, J=8.3,2.7,1.0 \mathrm{~Hz}, 1 \mathrm{H}), 4.84(\mathrm{~s}, 1 \mathrm{H})$, $4.81(\mathrm{~s}, 1 \mathrm{H}), 4.43(\mathrm{t}, J=6.8 \mathrm{~Hz}, 2 \mathrm{H}), 3.85(\mathrm{~s}, 3 \mathrm{H}), 2.48(\mathrm{t}, J=6.8 \mathrm{~Hz}, 2 \mathrm{H}), 1.81(\mathrm{~s}, 3 \mathrm{H})$.

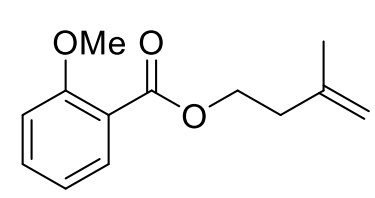

\section{3-methylbut-3-en-1-yl 2-methoxybenzoate (11)}

Following the general procedure A on a $0.6 \mathrm{mmol}$ scale using 2-methoxybenzoic acid, the desired product was obtained as a colorless oil (102 $\mathrm{mg}, 0.46 \mathrm{mmol}, 77 \%)$ after purification by flash chromatography (15\% EtOAc/hexanes). Spectroscopic data agreed with the literature. ${ }^{1} \mathrm{H}$ NMR $\left(500 \mathrm{MHz}, \mathrm{CDCl}_{3}\right) \delta 7.82-7.76(\mathrm{~m}, 1 \mathrm{H}), 7.50-7.43(\mathrm{~m}, 1 \mathrm{H}), 7.00-6.93(\mathrm{~m}, 2 \mathrm{H})$, $4.86-4.83(\mathrm{~m}, 1 \mathrm{H}), 4.82-4.80(\mathrm{~m}, 1 \mathrm{H}), 4.41(\mathrm{t}, J=6.8 \mathrm{~Hz}, 2 \mathrm{H}), 3.90(\mathrm{~s}, 3 \mathrm{H}), 2.47(\mathrm{t}, J=6.8 \mathrm{~Hz}, 2 \mathrm{H})$, $1.81(\mathrm{~s}, 3 \mathrm{H})$. 


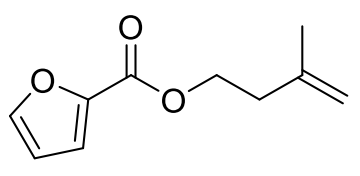

\section{3-methylbut-3-en-1-yl furan-2-carboxylate (1m)}

Following the general procedure $\mathrm{B}$ on a $1 \mathrm{mmol}$ scale using furan-2-carboxylic acid, the desired product was obtained as a colorless oil (142 $\mathrm{mg}, 0.79 \mathrm{mmol}, 79 \%)$ after purification by flash chromatography (5\% EtOAc/hexanes). IR (ATR, diamond), v = 2970, 1717, 1582, 1474, 1398, 1294, 1178, 1115, 1013, 885, $758 \mathrm{~cm}^{-1} ;{ }^{1} \mathrm{H}$ NMR $\left(500 \mathrm{MHz}, \mathrm{CDCl}_{3}\right) \delta 7.58(\mathrm{dd}, J=$ 1.8, $0.9 \mathrm{~Hz}, 1 \mathrm{H}), 7.17(\mathrm{dd}, J=3.4,0.9 \mathrm{~Hz}, 1 \mathrm{H}), 6.50$ (dd, $J=3.4,1.7 \mathrm{~Hz}, 1 \mathrm{H}), 4.83(\mathrm{~s}, 1 \mathrm{H}), 4.79$ (s, $1 \mathrm{H})$, $4.42(\mathrm{t}, J=6.9 \mathrm{~Hz}, 2 \mathrm{H}), 2.46(\mathrm{t}, J=6.9 \mathrm{~Hz}, 2 \mathrm{H}), 1.80(\mathrm{~s}, 3 \mathrm{H}) ;{ }^{13} \mathrm{C} \mathrm{NMR}\left(126 \mathrm{MHz}, \mathrm{CDCl}_{3}\right) \delta 158.7$, 146.3, 144.7, 141.5, 117.8, 112.5, 111.8, 63.2, 36.8, 22.6; HRMS-ESI calcd for $\mathrm{C}_{10} \mathrm{H}_{13} \mathrm{O}_{3}[\mathrm{M}+\mathrm{H}]^{+}$ 181.0859, found 181.0857.

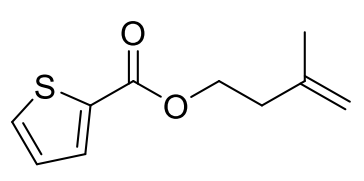

\section{3-methylbut-3-en-1-yl thiophene-2-carboxylate (1n)}

Following the general procedure B on a 1 mmol scale using thiophene-2carboxylic acid, the desired product was obtained as a colorless oil (139 mg, 0.71 mmol, 71\%) after purification by flash chromatography (5\% EtOAc/hexanes). Spectroscopic data agreed with the literature. ${ }^{4} \mathrm{H}$ NMR $\left(500 \mathrm{MHz}, \mathrm{CDCl}_{3}\right) \delta 7.79(\mathrm{dd}, J=3.7,1.3 \mathrm{~Hz}, 1 \mathrm{H}), 7.55(\mathrm{dd}, J=5.0,1.3 \mathrm{~Hz}$, $1 \mathrm{H}), 7.10(\mathrm{dd}, J=5.0,3.7 \mathrm{~Hz}, 1 \mathrm{H}), 4.86-4.82(\mathrm{~m}, 1 \mathrm{H}), 4.82-4.78(\mathrm{~m}, 1 \mathrm{H}), 4.41(\mathrm{t}, J=6.8 \mathrm{~Hz}, 2 \mathrm{H})$, $2.46(\mathrm{t}, J=6.7 \mathrm{~Hz}, 2 \mathrm{H}), 1.81-1.80(\mathrm{~m}, 3 \mathrm{H})$.<smiles>C=C(C)CCOC(=O)Cc1ccccc1</smiles>

\section{3-methylbut-3-en-1-yl 2-phenylacetate (10)}

the desired product was obtained as a colorless oil (194 mg, $0.95 \mathrm{mmol}, 95 \%)$ after purification by flash chromatography (10\% EtOAc/hexanes). IR (ATR, diamond), $v=3065,2968,1732,1497,1454,1242$, 1151, 1030, 891, 723 $\mathrm{cm}^{-1}$; ${ }^{1} \mathrm{H}$ NMR (400 MHz, $\left.\mathrm{CDCl}_{3}\right) \delta 7.35-7.18(\mathrm{~m}, 5 \mathrm{H}), 4.77(\mathrm{~s}, 1 \mathrm{H}), 4.69(\mathrm{~s}, 1 \mathrm{H})$, $4.20(\mathrm{t}, J=6.8 \mathrm{~Hz}, 2 \mathrm{H}), 3.60(\mathrm{~s}, 2 \mathrm{H}), 2.32(\mathrm{t}, J=6.8 \mathrm{~Hz}, 2 \mathrm{H}), 1.71(\mathrm{~s}, 3 \mathrm{H}) ;{ }^{13} \mathrm{C} \mathrm{NMR}\left(101 \mathrm{MHz}, \mathrm{CDCl}_{3}\right)$ $\delta 171.5,141.5,134.1,129.3,128.5,127.0,112.3,63.0,41.4,36.7,22.4 ;$ HRMS-ESI calcd for $\mathrm{C}_{13} \mathrm{H}_{17} \mathrm{O}_{2}$ $[\mathrm{M}+\mathrm{H}]^{+}$205.1223, found 205.1225.

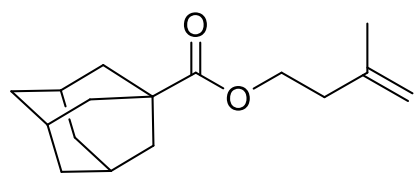

3-methylbut-3-en-1-yl (3r,5r,7r)-adamantane-1-carboxylate (1p)

Following the general procedure $\mathrm{B}$ on a $1 \mathrm{mmol}$ scale using $(3 r, 5 r, 7 r)$ adamantane-1-carboxylic acid, the desired product was obtained as a colorless

\footnotetext{
${ }^{4}$ Schevenels, F. T.; Shen, M.; Snyder, S. A. J. Am. Chem. Soc. 2017, 139, 6329-6337
} 
oil (92 mg, $0.37 \mathrm{mmol}, 37 \%$ ) after purification by flash chromatography (10\% EtOAc/hexanes). IR (ATR, diamond), $v=2905,2851,1726,1452,1325,1229,1070,889 \mathrm{~cm}^{-1} ;{ }^{1} \mathrm{H} \mathrm{NMR}\left(400 \mathrm{MHz}, \mathrm{CDCl}_{3}\right) \delta 4.81-$ $4.79(\mathrm{~m}, 1 \mathrm{H}), 4.73(\mathrm{dq}, J=2.0,1.0 \mathrm{~Hz}, 1 \mathrm{H}), 4.16(\mathrm{t}, J=6.7 \mathrm{~Hz}, 2 \mathrm{H}), 2.33(\mathrm{t}, J=6.7 \mathrm{~Hz}, 2 \mathrm{H}), 2.03-1.98$ $(\mathrm{m}, 3 \mathrm{H}), 1.89-1.86(\mathrm{~m}, 6 \mathrm{H}), 1.77-1.75(\mathrm{~m}, 3 \mathrm{H}), 1.73-1.69(\mathrm{~m}, 6 \mathrm{H}) ;{ }^{13} \mathrm{C} \mathrm{NMR}\left(101 \mathrm{MHz}, \mathrm{CDCl}_{3}\right) \delta$ 177.7, 141.9, 112.2, 62.2, 40.7, 38.8, 36.8, 36.5, 28.0, 22.5; HRMS-ESI calcd for $\mathrm{C}_{16} \mathrm{H}_{25} \mathrm{O}_{2}[\mathrm{M}+\mathrm{H}]^{+}$ 249.1849 , found 249.1850 .

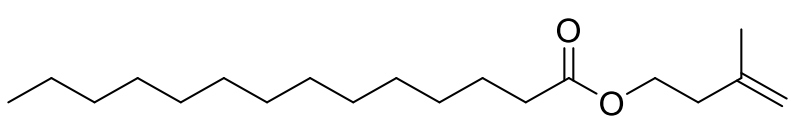

\section{3-methylbut-3-en-1-yl tetradecanoate (1q)}

Following the general procedure A on a $0.6 \mathrm{mmol}$ scale using myristic acid, the desired product was obtained as a colorless oil (156 mg, $0.53 \mathrm{mmol}, 88 \%$ ) after purification by flash chromatography (10\% EtOAc/hexanes). IR (ATR, diamond), $v=2955,2854,1736$, 1653, 1464, 1374, 1244, 1167, 1034, $891 \mathrm{~cm}^{-1} ;{ }^{1} \mathrm{H}$ NMR (500 MHz, $\left.\mathrm{CDCl}_{3}\right) \delta 4.80(1 \mathrm{H}, \mathrm{s}), 4.73(1 \mathrm{H}, \mathrm{s})$, $4.19(2 \mathrm{H}, \mathrm{t}, J=6.9 \mathrm{~Hz}), 2.34(2 \mathrm{H}, \mathrm{t}, J=6.9 \mathrm{~Hz}), 2.29(2 \mathrm{H}, \mathrm{t}, J=7.6 \mathrm{~Hz}), 1.76(3 \mathrm{H}, \mathrm{s}), 1.64-1.56(2 \mathrm{H}, \mathrm{m})$, 1.34-1.21 (20H, m), $0.88(3 \mathrm{H}, \mathrm{t}, J=6.9 \mathrm{~Hz}) ;{ }^{13} \mathrm{C} \mathrm{NMR}\left(126 \mathrm{MHz}, \mathrm{CDCl}_{3}\right) \delta 174.0,141.9,112.3,62.5$, 36.9, 34.5, 32.1, 29.8 (4C), 29.6, 29.5, 29.4, 29.3, 25.1, 22.8, 22.6, 14.3; HRMS-ESI calcd for $\mathrm{C}_{19} \mathrm{H}_{37} \mathrm{O}_{2}$ $[\mathrm{M}+\mathrm{H}]^{+}$297.2788, found 297.2785.

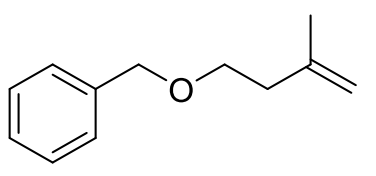

\section{(((3-methylbut-3-en-1-yl)oxy)methyl)benzene (1r)}

To a stirred suspension of sodium hydride (60\% suspension in oil, $800 \mathrm{mg}, 20$ mmol, 2,1 equiv.) in THF (40 mL, $0.25 \mathrm{M})$ at $0{ }^{\circ} \mathrm{C}$ was added 3-methyl-3-buten1-ol (1.0 mL, 9.9 mmol, 1 equiv.) dropwise. Benzyl bromide (1.17 mL, 9.9 mmol, 1 equiv.) was added and the mixture was stirred overnight at room temperature. The reaction was quenched with a saturated solution of $\mathrm{NH}_{4} \mathrm{Cl}$, extracted with EtOAc (2x). The organic layer was then dried with $\mathrm{Na}_{2} \mathrm{SO}_{4}$ and concentrated under reduced pressure. Purification by flash chromatography ( $5 \%$ EtOAc/hexanes) afforded the desired product $(1.45 \mathrm{~g}, 83 \%)$ as a colorless oil. Spectroscopic data agreed with the literature. ${ }^{5}{ }^{1} \mathrm{H}$ NMR (500 MHz, $\left.\mathrm{CDCl}_{3}\right) \delta 7.37-7.32(\mathrm{~m}, 4 \mathrm{H}), 7.32$ - $7.25(\mathrm{~m}, 1 \mathrm{H}), 4.82-4.76(\mathrm{~m}, 1 \mathrm{H}), 4.77-4.72(\mathrm{~m}$, 1H), $4.53(\mathrm{~s}, 2 \mathrm{H}), 3.59(\mathrm{t}, J=6.9 \mathrm{~Hz}, 2 \mathrm{H}), 2.35(\mathrm{t}, 2 \mathrm{H}), 1.77-1.73(\mathrm{~m}, 3 \mathrm{H})$.

\footnotetext{
${ }^{5}$ Cleary, P. A.; Woerpel, K. A. Org. Lett., 2005, 7, 5531-5533
} 


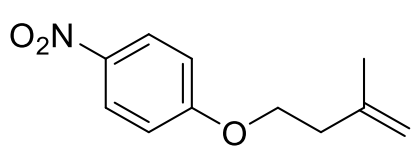

\section{1-((3-methylbut-3-en-1-yl)oxy)-4-nitrobenzene (1s)}

A solution of 4-nitrophenol (139 mg, $1 \mathrm{mmol}, 1$ equiv.), 3-methyl-3-buten-1-

ol (0.12 mL, $1.2 \mathrm{mmol}, 1.2$ equiv.), triphenylphosphine (315 mg, $1.2 \mathrm{mmol}, 1.2$ equiv.), diisopropyl azodicarboxylate (DIAD, $0.24 \mathrm{~mL}, 1.2 \mathrm{mmol}, 1.2$ equiv.) in THF (9 mL, $0.11 \mathrm{M}$ ) was heated for 1.5 hours at reflux, then the crude mixture was concentrated under reduced pressure. Purification by flash chromatography (10\% EtOAc/hexanes) afforded the desired product (170 $\mathrm{mg}, 0.82$ mmol, 82\%) as a colorless oil. Spectroscopic data agreed with the literature. ${ }^{6} \mathrm{H} \mathrm{NMR}\left(500 \mathrm{MHz}, \mathrm{CDCl}_{3}\right)$ $\delta 8.24-8.17(\mathrm{~m}, 1 \mathrm{H}), 6.99-6.92(\mathrm{~m}, 1 \mathrm{H}), 4.90-4.85(\mathrm{~m}, 1 \mathrm{H}), 4.83-4.79(\mathrm{~m}, 1 \mathrm{H}), 4.17(\mathrm{t}, J=6.8 \mathrm{~Hz}$ $1 \mathrm{H}), 2.54(\mathrm{t}, J=6.9 \mathrm{~Hz}, 0 \mathrm{H}), 1.84-1.80(\mathrm{~m}, 4 \mathrm{H})$.

TsO $\begin{aligned} & \text { 3-methylbut-3-en-1-yl 4-methylbenzenesulfonate (1t) } \\ & \text { 3-methyl-3-buten-1-ol }(61 \mu \mathrm{L}, 0.6 \mathrm{mmol}, 1 \text { equiv. }) \text { was added to } \mathrm{CH}_{2} \mathrm{Cl}_{2}(2 \mathrm{~mL}, 0.3 \mathrm{M})\end{aligned}$ at $0{ }^{\circ} \mathrm{C}$, followed by DMAP ( $88 \mathrm{mg}, 0.72 \mathrm{mmol}, 1.2$ equiv.) and $\mathrm{TsCl}$ (137 $\mathrm{mg}, 1.2$ equiv.). The reaction was warmed to room temperature and stirred overnight. Aq. $\mathrm{HCl}(1 \mathrm{M})$ was added to the mixture, which was extracted with $\mathrm{CH}_{2} \mathrm{Cl}_{2}(3 \mathrm{x})$. The organic layers were combined, washed with aq. saq. $\mathrm{NaHCO}_{3}$ and brine, dried over $\mathrm{Na}_{2} \mathrm{SO}_{4}$, filtered and concentrated under reduced pressure. Purification by flash chromatography (10\% EtOAc/hexanes) afforded the desired product (128 $\mathrm{mg}, 89 \%)$ as a colorless oil. Spectroscopic data agreed with the literature. ${ }^{1}{ }^{1} \mathrm{H} \mathrm{NMR}\left(400 \mathrm{MHz}, \mathrm{CDCl}_{3}\right) \delta 7.83-7.76(\mathrm{~m}, 2 \mathrm{H}), 7.39-$ $7.31(\mathrm{~m}, 2 \mathrm{H}), 4.79(\mathrm{~s}, 1 \mathrm{H}), 4.68(\mathrm{~s}, 1 \mathrm{H}), 4.13(\mathrm{t}, J=6.9 \mathrm{~Hz}, 2 \mathrm{H}), 2.45(\mathrm{~s}, 3 \mathrm{H}), 2.39-2.31(\mathrm{~m}, 2 \mathrm{H}), 1.66$ $(\mathrm{s}, 3 \mathrm{H})$.

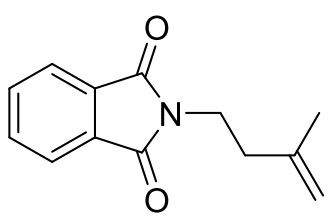

\section{2-(3-methylbut-3-en-1-yl)isoindoline-1,3-dione (1u)}

Following Siu's procedure ${ }^{7}$ using 3-methylbut-3-en-1-yl 4-methylbenzenesulfonate (144 $\mathrm{mg}, 0.6 \mathrm{mmol})$, the desired product was obtained as a colorless oil ( $65 \mathrm{mg}, 0.3$ mmol, 50\%) after purification by flash chromatography $\left(100 \% \quad \mathrm{CH}_{2} \mathrm{Cl}_{2}\right)$. Spectroscopic data agreed with the literature. ${ }^{4}{ }^{1} \mathrm{H} \mathrm{NMR}\left(400 \mathrm{MHz}, \mathrm{CDCl}_{3}\right) \delta 7.96-7.78(\mathrm{~m}, 2 \mathrm{H}), 7.77-$ $7.66(\mathrm{~m}, 2 \mathrm{H}), 4.74(\mathrm{~m}, 1 \mathrm{H}), 4.71-4.64(\mathrm{~m}, 1 \mathrm{H}), 3.83(\mathrm{t}, J=7.0 \mathrm{~Hz}, 2 \mathrm{H}), 2.40(\mathrm{t}, J=7.1 \mathrm{~Hz}, 2 \mathrm{H}), 1.81(\mathrm{~s}$, $3 \mathrm{H})$.

\footnotetext{
${ }^{6}$ Lu, Z.; Bajwa, B. S.; Otome, O. E.; Hammond, G. B.; Xu, B. Green Chem., 2019, 21, 2224-2228

${ }^{7}$ Siu, J. C.; Parry, J. B.; Lin, S. J. Am. Chem. Soc. 2019, 141, 2825-2831
} 


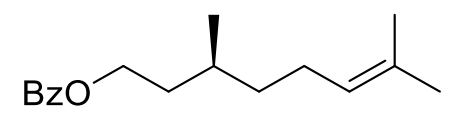

\section{(S)-3,7-dimethyloct-6-en-1-yl benzoate (1v)}

0.84 mmol, 1.4 equiv.) were added to $\mathrm{CH}_{2} \mathrm{Cl}_{2}(3 \mathrm{~mL}, 0.2 \mathrm{M})$. The mixture was cooled down to $0{ }^{\circ} \mathrm{C}$ and benzoyl chloride ( $84 \mu \mathrm{L}, 0.72 \mathrm{mmol}, 1.2$ equiv.) was added. The mixture was stirred overnight at room temperature. $10 \%$ aq. $\mathrm{HCl}$ was then added, and the organic layer was washed with water. The organic layer was then dried with $\mathrm{Na}_{2} \mathrm{SO}_{4}$ and concentrated under reduced pressure. Purification by flash chromatography (5\% EtOAc/hexanes) afforded the desired product (135 $\mathrm{mg}, 0.52 \mathrm{mmol}, 86 \%$ ) as a colorless oil. Spectroscopic data agreed with the literature. ${ }^{8}{ }^{1} \mathrm{H}$ NMR $\left(500 \mathrm{MHz}, \mathrm{CDCl}_{3}\right) \delta 8.07-8.01(\mathrm{~m}$, 2H), $7.59-7.51(\mathrm{~m}, 1 \mathrm{H}), 7.48-7.40(\mathrm{~m}, 2 \mathrm{H}), 5.14-5.06(\mathrm{~m}, 1 \mathrm{H}), 4.43-4.30(\mathrm{~m}, 2 \mathrm{H}), 2.09-1.93(\mathrm{~m}$, $2 \mathrm{H}), 1.87-1.77(\mathrm{~m}, 1 \mathrm{H}), 1.70-1.54(\mathrm{~m}, 8 \mathrm{H}), 1.46-1.35(\mathrm{~m}, 1 \mathrm{H}), 1.30-1.18(\mathrm{~m}, 1 \mathrm{H}), 0.97(\mathrm{~d}, J=6.6$ $\mathrm{Hz}, 3 \mathrm{H})$.

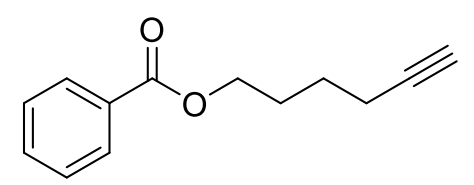

\section{Hex-5-yn-1-yl benzoate (S1)}

Hex-5-yn-1-ol (392 mg, 4 mmol, 1 equiv.) and $\mathrm{Et}_{3} \mathrm{~N}$ (0.78 mL, $5.6 \mathrm{mmol}, 1.4$ equiv.) were added to $\mathrm{CH}_{2} \mathrm{Cl}_{2}(20 \mathrm{~mL}, 0.2 \mathrm{M})$. The mixture was cooled down to $0{ }^{\circ} \mathrm{C}$ and benzoyl chloride $(0.56 \mathrm{~mL}, 4.8 \mathrm{mmol}, 1.2$ equiv.) was added. The mixture was stirred overnight at room temperature. $10 \%$ aq. $\mathrm{HCl}$ was then added, and the organic layer was washed with water. The organic layer was then dried with $\mathrm{Na}_{2} \mathrm{SO}_{4}$ and concentrated under reduced pressure. Purification by flash chromatography (5\% EtOAc/hexanes) afforded the desired product ( $809 \mathrm{mg}, 4 \mathrm{mmol}$, $>99 \%$ ) as a colorless oil. Spectroscopic data agreed with the literature. ${ }^{9}{ }^{1} \mathrm{H}$ NMR $\left(400 \mathrm{MHz}, \mathrm{CDCl}_{3}\right) \delta$ $8.05-8.02(\mathrm{~m}, 2 \mathrm{H}), 7.60-7.52(\mathrm{~m}, 1 \mathrm{H}), 7.49-7.40(\mathrm{~m}, 2 \mathrm{H}), 4.35(\mathrm{t}, J=6.4 \mathrm{~Hz}, 2 \mathrm{H}), 2.29(\mathrm{td}, J=7.0$, $2.7 \mathrm{~Hz}, 2 \mathrm{H}), 2.00-1.87(\mathrm{~m}, 3 \mathrm{H}), 1.75-1.68(\mathrm{~m}, 2 \mathrm{H})$.

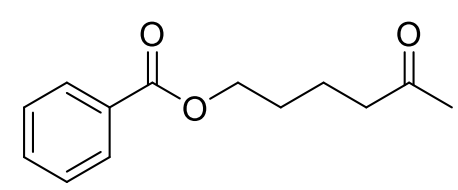

\section{5-oxohexyl benzoate (S2)}

Following Cai's procedures, ${ }^{10}$ hex-5-yn-1-yl benzoate (S1, 405 mg, 2 mmol, 1 equiv.) was added to a solution of p-toluenesulfonic acid monohydrate (380 mg, 2 mmol, 1 equiv.) and acetic acid $(1 \mathrm{~mL})$ in DCE $(2 \mathrm{~mL})$. The reaction was then sealed and stirred at $100{ }^{\circ} \mathrm{C}$ for 18 hours in an oil bath. Saturated aqueous $\mathrm{NaHCO}_{3}$ was added to quench the reaction and then

\footnotetext{
${ }^{8}$ Wommack, A. J.; Kingsbury, J. S. J. Org. Chem. 2013, 78, 10573-10587

${ }^{9}$ Atobe, S.; Masuno, H.; Sonoda, M.; Suzuki, Y.; Shinohara, H.; Shibata, S.; Ogawa, A. Tetrahedron Lett. 2012, 53, $1764-$ 1767

${ }^{10}$ H. Liu, Y. Wei, C. Cai, Synlett, 2016, 27, 2378-2383
} 
extracted with $\mathrm{CH}_{2} \mathrm{Cl}_{2}(3 \mathrm{x})$. The organic layer was then dried with $\mathrm{Na}_{2} \mathrm{SO}_{4}$ and concentrated under reduced pressure. Purification by flash chromatography (5\% EtOAc/hexanes) afforded the desired product (160 $\mathrm{mg}, 0.7 \mathrm{mmol}, 36 \%)$ as a colorless oil. Spectroscopic data agreed with the literature. ${ }^{11}{ }^{1} \mathrm{H} \mathrm{NMR}(400 \mathrm{MHz}$, $\left.\mathrm{CDCl}_{3}\right) \delta 8.09-8.00(\mathrm{~m}, 2 \mathrm{H}), 7.61-7.51(\mathrm{~m}, 1 \mathrm{H}), 7.49-7.40(\mathrm{~m}, 2 \mathrm{H}), 4.33(\mathrm{t}, J=6.0 \mathrm{~Hz}, 2 \mathrm{H}), 2.52(\mathrm{t}$, $J=6.8 \mathrm{~Hz}, 2 \mathrm{H}), 2.16(\mathrm{~s}, 3 \mathrm{H}), 1.83-1.70(\mathrm{~m}, 4 \mathrm{H})$.

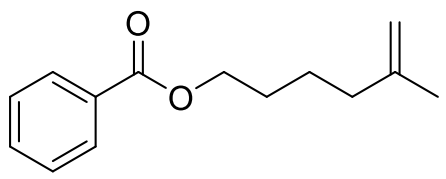

\section{5-methylhex-5-en-1-yl benzoate (1w).}

Potassium tert-butoxide (101 $\mathrm{mg}, \quad 0.9 \mathrm{mmol}, \quad 1.5$ equiv.) and methyltriphenylphosphonium bromide ( $322 \mathrm{mg}, 0.9 \mathrm{mmol}, 1.5$ equiv.) were added to $\mathrm{Et}_{2} \mathrm{O}(1 \mathrm{~mL})$. The mixture was stirred at room temperature for 30 minutes. 5-oxohexyl benzoate (S2, $132 \mathrm{mg}, 0.6 \mathrm{mmol}, 1$ equiv.) was dissolved in $0.5 \mathrm{~mL}$ of $\mathrm{Et}_{2} \mathrm{O}$, added to the mixture and stirred for 1.5 hours at $40^{\circ} \mathrm{C}$ in an oil bath. Water was added to quench the reaction and the organic layer was washed with water. The organic layer was then dried with $\mathrm{Na}_{2} \mathrm{SO}_{4}$ and concentrated under reduced pressure. Purification by flash chromatography (5\% EtOAc/hexanes) afforded the desired product ( $80 \mathrm{mg}, 0.37$ mmol, $61 \%)$ as a colorless oil. Spectroscopic data agreed with the literature. ${ }^{12}{ }^{1} \mathrm{H}$ NMR $\left(400 \mathrm{MHz}, \mathrm{CDCl}_{3}\right)$ $\delta 8.09-8.00(\mathrm{~m}, 2 \mathrm{H}), 7.61-7.51(\mathrm{~m}, 1 \mathrm{H}), 7.49-7.39(\mathrm{~m}, 2 \mathrm{H}), 4.77-4.70(\mathrm{~m}, 1 \mathrm{H}), 4.73-4.67(\mathrm{~m}, 1 \mathrm{H})$, $4.34(\mathrm{t}, J=6.6 \mathrm{~Hz}, 2 \mathrm{H}), 2.09$ (t, $J=7.6 \mathrm{~Hz}, 2 \mathrm{H}), 1.84-1.72(\mathrm{~m}, 2 \mathrm{H}), 1.76-1.70(\mathrm{~m}, 3 \mathrm{H}), 1.66-1.56$ $(\mathrm{m}, 2 \mathrm{H})$.

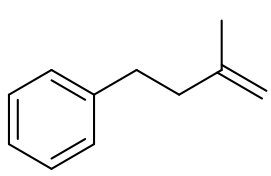

\section{(3-methylbut-3-en-1-yl)benzene (1x).}

Potassium tert-butoxide (168 mg, 1.5 mmol, 1.5 equiv. $)$ and methyltriphenylphosphonium bromide $(535.8 \mathrm{mg}, 1.5 \mathrm{mmol}, 1.5$ equiv.) were added to $\mathrm{Et}_{2} \mathrm{O}(2 \mathrm{~mL})$. The mixture was stirred at room temperature for 30 minutes. 4-phenylbutan-2-one (148 mg, $1 \mathrm{mmol}, 1$ equiv.) was dissolved in $1 \mathrm{~mL}$ of $\mathrm{Et}_{2} \mathrm{O}$, added to the mixture and stirred for 1.5 hours at $40{ }^{\circ} \mathrm{C}$ in an oil bath. Water was added to quench the reaction and the organic layer was washed with water. The organic layer was then dried with $\mathrm{Na}_{2} \mathrm{SO}_{4}$ and concentrated under reduced pressure. Purification by flash chromatography (100\% hexanes) afforded the desired product (126 mg, $86 \mathrm{mmol}, 86 \%)$ as a colorless oil. Spectroscopic data agreed with the literature. ${ }^{13}{ }^{1} \mathrm{H}$ NMR $\left(400 \mathrm{MHz}, \mathrm{CDCl}_{3}\right) \delta 7.31-7.26(\mathrm{~m}, 2 \mathrm{H}), 7.22$

\footnotetext{
${ }^{11}$ Tachinami, T.; Nishimura, T.; Ushimaru, R.; Noyori, R.; Naka, H. J. Am. Chem. Soc. 2013, 135, 50-53

${ }^{12}$ Murakami, M.; Amii, H.; Shigeto, K.; Ito, Y. J. Am. Chem. Soc. 1996, 118, 8285-8290

${ }^{13}$ Lebel, H.; Guay, D.; Paquet, V.; Huard, K. Org. Lett. 2004, 18, 3047-3050
} 
$-7.14(\mathrm{~m}, 3 \mathrm{H}), 4.75-4.73(\mathrm{~m}, 1 \mathrm{H}), 4.72-4.70(\mathrm{~m}, 1 \mathrm{H}), 2.78-2.73(\mathrm{~m}, 2 \mathrm{H}), 2.35-2.29(\mathrm{~m}, 2 \mathrm{H}), 1.78$ $-1.77(\mathrm{~m}, 3 \mathrm{H})$.

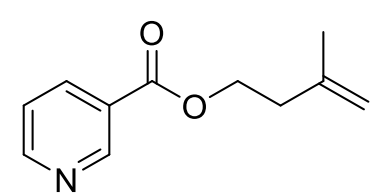

\section{3-methylbut-3-en-1-yl nicotinate (1y)}

Following the general procedure B on a $0.654 \mathrm{mmol}$ scale and using nicotinic acid, the desired product (112 $\mathrm{mg}, 0.59 \mathrm{mmol}, 90 \%)$ was obtained as a colorless oil after purification by flash chromatography (40\% EtOAc/hexanes). Spectroscopic data agreed with the literature. ${ }^{14}{ }^{1} \mathrm{H}$ NMR (400 MHz, CDCl $) \delta 9.23(\mathrm{~s}, 1 \mathrm{H}), 8.78(\mathrm{~d}, J=3.7 \mathrm{~Hz}, 1 \mathrm{H}), 8.39(\mathrm{~m}, 1 \mathrm{H}), 7.39$ (dd, $J=8.0,4.8 \mathrm{~Hz}, 1 \mathrm{H}), 4.86(\mathrm{~s}, 1 \mathrm{H}), 4.82(\mathrm{~s}, 1 \mathrm{H}), 4.48(\mathrm{t}, J=6.8 \mathrm{~Hz}, 2 \mathrm{H}), 2.50(\mathrm{t}, J=6.7 \mathrm{~Hz}, 2 \mathrm{H}), 1.82(\mathrm{~s}$, $3 \mathrm{H})$.<smiles>C=C(C)COC(=O)c1ccccc1</smiles>

\section{2-methylallyl benzoate (1z).}

2-methylprop-2-en-1-ol (85 $\mu \mathrm{L}, 1 \mathrm{mmol}, 1$ equiv.) and $\mathrm{Et}_{3} \mathrm{~N}(140 \mu \mathrm{L}, 1.2 \mathrm{mmol}, 1.2$ equiv.) were added to $\mathrm{CH}_{2} \mathrm{Cl}_{2}(5 \mathrm{~mL}, 0.2 \mathrm{M})$. The mixture was cooled down to $0{ }^{\circ} \mathrm{C}$ and benzoyl chloride (200 $\mu \mathrm{L}, 1.4 \mathrm{mmol}, 1.4$ equiv.) was added. The mixture was stirred overnight at room temperature. $10 \%$ aq. $\mathrm{HCl}$ was then added, and the organic layer was washed with water. The organic layer was then dried with $\mathrm{Na}_{2} \mathrm{SO}_{4}$ and concentrated under reduced pressure. Purification by flash chromatography (5\% EtOAc/hexanes) afforded the desired product (176 $\mathrm{mg}, 1 \mathrm{mmol}$, >99\%) as a colorless oil. Spectroscopic data agreed with the literature. ${ }^{15}{ }^{1} \mathrm{H}$ NMR $\left(500 \mathrm{MHz}, \mathrm{CDCl}_{3}\right) \delta 8.12-8.06$ (m, 2H), $7.62-7.54(\mathrm{~m}, 1 \mathrm{H}), 7.50-7.43(\mathrm{~m}, 2 \mathrm{H}), 5.12-5.07(\mathrm{~m}, 1 \mathrm{H}), 5.03-4.98(\mathrm{~m}, 1 \mathrm{H}), 4.77(\mathrm{~s}, 3 \mathrm{H})$, $1.86-1.85(\mathrm{~m}, 3 \mathrm{H})$.

\section{Hydrofluorination reaction}

General procedure C: The alkene (1 equiv.) was added to $\mathrm{CH}_{2} \mathrm{Cl}_{2}(0.2 \mathrm{M})$ in a polystyrene conical tube. Triethylamine trihydrofluoride (5.0 equiv.) and methanesulfonic acid (5.0 equiv.) were added and the mixture was stirred at room temperature. Upon reaction completion, the mixture was washed with aq. sat. $\mathrm{NaHCO}_{3}$, dried over $\mathrm{Na}_{2} \mathrm{SO}_{4}$, filtered and concentrated under reduced pressure. A known amount of 2-

\footnotetext{
${ }^{14}$ Lu, Z.; Zeng, X.; Hammond, G. B.; Xu, B. J. Am. Chem. Soc. 2017, 139, 18202-18205

${ }^{15}$ Mamone, P.; Grünberg, M. F.; Fromm, A.; Khan, B. A.; Gooßen, L. J. Org. Lett., 2012, 14, 3716-3719
} 
fluoro-4-nitrotoluene was added and a NMR yield was determined by ${ }^{19} \mathrm{~F}$ NMR. If applicable, purification by flash chromatography afforded the final product.

LF $^{\mathrm{F}}$ 3-fluoro-3-methylbutyl benzoate (2a)

Following general procedure $\mathrm{C}$ using 3-methylbut-3-en-1-yl benzoate (1a, $38 \mathrm{mg}, 0.2$ $\mathrm{mmol})$, the desired product was obtained after 4 hours (78\% NMR yield; estimated by ${ }^{19} \mathrm{~F}$ NMR analysis of the crude mixture after workup using 2-fluoro-4-nitrotoluene as the internal standard). ${ }^{19}$ F NMR (470 $\left.\mathrm{MHz}, \mathrm{CDCl}_{3}\right) \delta-138.2(\mathrm{~m}, 1 \mathrm{~F})^{14}$<smiles>CC(C)(C)CCOC(=O)c1ccc(C(C)(C)C)cc1</smiles>

\section{3-fluoro-3-methylbutyl 4-(tert-butyl)benzoate (2b)}

Following general procedure C using 3-methylbut-3-en-1-yl 4-(tertbutyl)benzoate (1b, $49 \mathrm{mg}, 0.2 \mathrm{mmol})$ for 24 hours, analysis of the crude

mixture indicated a 63\% NMR yield. The desired product was obtained as a colorless oil. (32 mg, 0.12 mmol, 60\%) after purification by flash chromatography (50\% toluene/hexanes). IR (ATR, diamond), $v=$ 2964, 1720, 1610, 1462, 1275, 1188, 1117, 1016, 854, $775 \mathrm{~cm}^{-1} ;{ }^{1} \mathrm{H}$ NMR $\left(500 \mathrm{MHz}, \mathrm{CDCl}_{3}\right) \delta 7.98-$ $7.94(\mathrm{~m}, 2 \mathrm{H}), 7.48-7.43(\mathrm{~m}, 2 \mathrm{H}), 4.46(\mathrm{t}, J=6.8 \mathrm{~Hz}, 2 \mathrm{H}), 2.12(\mathrm{dt}, J=19.3,6.8 \mathrm{~Hz}, 2 \mathrm{H}), 1.45(\mathrm{~d}, J=$ $21.5 \mathrm{~Hz}, 6 \mathrm{H}), 1.34$ (s, 9H); ${ }^{13} \mathrm{C}$ NMR $\left(101 \mathrm{MHz}, \mathrm{CDCl}_{3}\right) \delta 166.5,156.6,129.4,127.4,125.4,94.3(\mathrm{~d}, J=$ $165.9 \mathrm{~Hz}), 60.7(\mathrm{~d}, J=6.2 \mathrm{~Hz}), 39.9$ (d, $J=23.3 \mathrm{~Hz}), 35.1,31.1,27.1(\mathrm{~d}, J=24.7 \mathrm{~Hz}) ;{ }^{19} \mathrm{~F}$ NMR $(470$ $\left.\mathrm{MHz}, \mathrm{CDCl}_{3}\right) \delta-137.9(\mathrm{~m}, 1 \mathrm{~F})$; HRMS-ESI calcd for $\mathrm{C}_{16} \mathrm{H}_{24} \mathrm{FO}_{2}[\mathrm{M}+\mathrm{H}]^{+}$267.1755, found 267.1754.

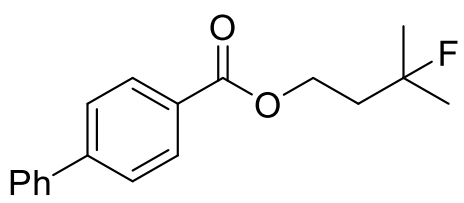

\section{3-fluoro-3-methylbutyl [1,1'-biphenyl]-4-carboxylate (2c)}

Following general procedure C using 3-methylbut-3-en-1-yl [1,1'biphenyl]-4-carboxylate (1c, $53 \mathrm{mg}, 0.2 \mathrm{mmol})$ for 9 hours, analysis of the crude mixture indicated a 74\% NMR yield. The desired product was obtained as a colorless oil (45 mg, 0,156 mmol, 78\%) after purification by flash chromatography (toluene). On a $1 \mathrm{mmol}$ scale: $199 \mathrm{mg}, 0.7$ mmol, $75 \%$ NMR yield, $70 \%$ isolated. Spectroscopic data agreed with the literature. ${ }^{1}{ }^{1} \mathrm{H}$ NMR (500 $\left.\mathrm{MHz} \mathrm{CDCl}_{3}\right) \delta 8.10(\mathrm{~d}, J=8.1 \mathrm{~Hz}, 2 \mathrm{H}), 7.65(\mathrm{~d}, J=8.1 \mathrm{~Hz}, 2 \mathrm{H}), 7.61(\mathrm{~d}, J=7.6 \mathrm{~Hz}, 2 \mathrm{H}), 7.46(\mathrm{t}, J=$ $7.5 \mathrm{~Hz}, 2 \mathrm{H}), 7.39(\mathrm{t}, J=7.3 \mathrm{~Hz}, 1 \mathrm{H}), 4.50(\mathrm{t}, J=6.8 \mathrm{~Hz}, 2 \mathrm{H}), 2.14(\mathrm{dt}, J=19.4,6.8 \mathrm{~Hz}, 2 \mathrm{H}), 1.46(\mathrm{~d}, J=$ $21.4 \mathrm{~Hz}, 6 \mathrm{H}) ;{ }^{19} \mathrm{~F}$ NMR $\left(470 \mathrm{MHz}, \mathrm{CDCl}_{3}\right) \delta-138.1$ (m, 1F). 
3-methylbut-3-en-1-yl [1,1'-biphenyl]-4-carboxylate (1c, $266 \mathrm{mg}, 1 \mathrm{mmol}, 1$ equiv.) was added to $\mathrm{CH}_{2} \mathrm{Cl}_{2}$ ( $5 \mathrm{~mL}, 0.2 \mathrm{M}$ ). Triethylamine trihydrofluoride ( $806 \mathrm{mg}, 5 \mathrm{mmol}, 5.0$ equiv.) and methanesulfonic acid (481 mg, $5 \mathrm{mmol}, 5.0$ equiv.) were added and the mixture was stirred 9 hours at room temperature. The mixture was washed with aq. sat. $\mathrm{NaHCO}_{3}$, dried over $\mathrm{Na}_{2} \mathrm{SO}_{4}$, filtered and concentrated under reduced pressure. Analysis of the crude mixture indicated a 75\% NMR yield. The desired product was obtained as a colorless oil (199 $\mathrm{mg}, 0,7 \mathrm{mmol}, 70 \%$ ) after purification by flash chromatography (toluene). Spectroscopic data agreed with the literature. ${ }^{1} \mathrm{H}$ NMR $\left(500 \mathrm{MHz}, \mathrm{CDCl}_{3}\right) \delta 8.10(\mathrm{~d}, J=8.1 \mathrm{~Hz}, 2 \mathrm{H})$, $7.65(\mathrm{~d}, J=8.1 \mathrm{~Hz}, 2 \mathrm{H}), 7.61(\mathrm{~d}, J=7.6 \mathrm{~Hz}, 2 \mathrm{H}), 7.46(\mathrm{t}, J=7.5 \mathrm{~Hz}, 2 \mathrm{H}), 7.39(\mathrm{t}, J=7.3 \mathrm{~Hz}, 1 \mathrm{H}), 4.50$ $(\mathrm{t}, J=6.8 \mathrm{~Hz}, 2 \mathrm{H}), 2.14(\mathrm{dt}, J=19.4,6.8 \mathrm{~Hz}, 2 \mathrm{H}), 1.46(\mathrm{~d}, J=21.4 \mathrm{~Hz}, 6 \mathrm{H}) ;{ }^{19} \mathrm{~F} \mathrm{NMR}\left(470 \mathrm{MHz}, \mathrm{CDCl}_{3}\right)$ $\delta-138.1(\mathrm{~m}, 1 \mathrm{~F})$.

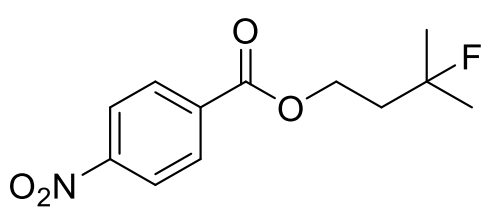

\section{3-fluoro-3-methylbutyl 4-nitrobenzoate (2d)}

Following general procedure $\mathrm{C}$ using 3-methylbut-3-en-1-yl 4nitrobenzoate (1g, $47 \mathrm{mg}, 0.2 \mathrm{mmol}$ ) for 30 hours, analysis of the crude mixture indicated a $63 \%$ NMR yield. The desired product was obtained as a colorless oil. (31 mg, $0.12 \mathrm{mmol}, 61 \%$ ) after purification by flash chromatography (100\% toluene). Spectroscopic data agreed with the literature. ${ }^{16}{ }^{1} \mathrm{H}$ NMR $\left(500 \mathrm{MHz}, \mathrm{CDCl}_{3}\right) \delta 8.32-8.27(\mathrm{~m}, 2 \mathrm{H}), 8.23$ $-8.19(\mathrm{~m}, 2 \mathrm{H}), 4.55(\mathrm{t}, J=6.8 \mathrm{~Hz}, 2 \mathrm{H}), 2.15(\mathrm{dt}, J=19.9,6.8 \mathrm{~Hz}, 2 \mathrm{H}), 1.46(\mathrm{~d}, J=21.4 \mathrm{~Hz}, 6 \mathrm{H}) ;{ }^{19} \mathrm{~F}$ $\operatorname{NMR}\left(470 \mathrm{MHz}, \mathrm{CDCl}_{3}\right) \delta-139.0(\mathrm{~m}, 1 \mathrm{~F})$.

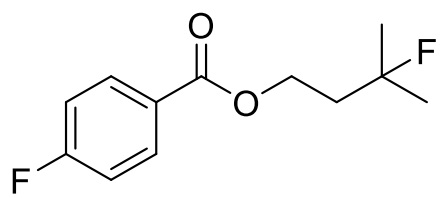

\section{3-fluoro-3-methylbutyl 4-fluorobenzoate (2e)}

Following general procedure $\mathrm{C}$ using 3-methylbut-3-en-1-yl 4fluorobenzoate (1e, $42 \mathrm{mg}, 0.2 \mathrm{mmol}$ ) for 4 hours, analysis of the crude mixture indicated a 82\% NMR yield. The desired product was obtained as a colorless oil (32 mg, 0.14 $\mathrm{mmol}, 70 \%)$ after purification by flash chromatography (50\% toluene/hexane). Spectroscopic data agreed with the literature. ${ }^{1}{ }^{1} \mathrm{H}$ NMR $\left(500 \mathrm{MHz}, \mathrm{CDCl}_{3}\right) \delta 8.08-8.02(\mathrm{~m}, 2 \mathrm{H}), 7.15-7.07(\mathrm{~m}, 2 \mathrm{H}), 4.47(\mathrm{t}, J=$ $6.8 \mathrm{~Hz}, 2 \mathrm{H}), 2.12(\mathrm{dt}, J=19.6,6.8 \mathrm{~Hz}, 2 \mathrm{H}), 1.45(\mathrm{~d}, J=21.4 \mathrm{~Hz}, 6 \mathrm{H}) ;{ }^{19} \mathrm{~F} \mathrm{NMR}\left(470 \mathrm{MHz}, \mathrm{CDCl}_{3}\right) \delta-$ $105.7(\mathrm{tt}, J=9.1,5.6 \mathrm{~Hz}, 1 \mathrm{~F}),-138.4(\mathrm{~m}, 1 \mathrm{~F})$.

\footnotetext{
${ }^{16}$ Chen, H.; Liu, Z.; Lv, Y.; Tan, X.; Shen, H.; Yu, H.-Z.; Li, C. Angew. Chem. Int. Ed. 2017, 56, 15411-15415.
} 


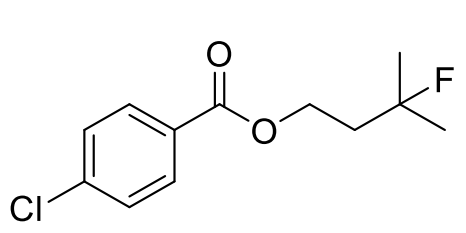

\section{3-fluoro-3-methylbutyl 4-chlorobenzoate (2f)}

Following general procedure $\mathrm{C}$ using 3-methylbut-3-en-1-yl 4chlorobenzoate (1f, $45 \mathrm{mg}, 0.2 \mathrm{mmol}$ ) for 24 hours, analysis of the crude mixture indicated a $66 \%$ NMR yield. The desired product was obtained as a colorless oil. (32 mg, 0,13 mmol, 64\%) after purification by flash chromatography ( $50 \%$ toluene/hexanes). Spectroscopic data agreed with the literature. ${ }^{16}{ }^{1} \mathrm{H}$ NMR $\left(500 \mathrm{MHz}, \mathrm{CDCl}_{3}\right) \delta 8.00-7.94(\mathrm{~m}, 2 \mathrm{H}), 7.45$ $-7.37(\mathrm{~m}, 2 \mathrm{H}), 4.48(\mathrm{t}, J=6.8 \mathrm{~Hz}, 2 \mathrm{H}), 2.12(\mathrm{dt}, J=19.6,6.8 \mathrm{~Hz}, 2 \mathrm{H}), 1.45(\mathrm{~d}, J=21.4 \mathrm{~Hz}, 6 \mathrm{H}) ;{ }^{19} \mathrm{~F}$ $\operatorname{NMR}\left(470 \mathrm{MHz}, \mathrm{CDCl}_{3}\right) \delta-138.5(\mathrm{~m}, 1 \mathrm{~F})$.

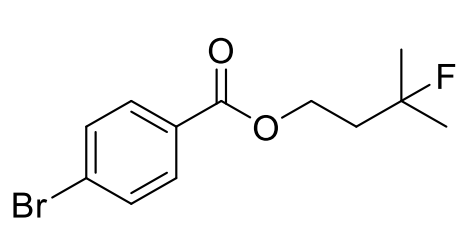

\section{3-fluoro-3-methylbutyl 4-bromobenzoate (2g)}

Following general procedure $\mathrm{C}$ using 3-methylbut-3-en-1-yl 4bromobenzoate (1g, $54 \mathrm{mg}, 0.2 \mathrm{mmol})$ for 24 hours, analysis of the crude mixture indicated a $65 \%$ NMR yield. The desired product was obtained as a colorless oil. (31 mg, $0.11 \mathrm{mmol}, 54 \%$ ) after purification by flash chromatography (50\% toluene/hexanes). IR (ATR, diamond), $v=2982,2930,1717,1591,1398,1269,1173,1103,1013,754 \mathrm{~cm}^{-1} ;{ }^{1} \mathrm{H}$ NMR (500 $\left.\mathrm{MHz}, \mathrm{CDCl}_{3}\right) \delta 7.92-7.85(\mathrm{~m}, 2 \mathrm{H}), 7.61-7.54(\mathrm{~m}, 2 \mathrm{H}), 4.48(\mathrm{t}, J=6.8 \mathrm{~Hz}, 2 \mathrm{H}), 2.12(\mathrm{dt}, J=19.6,6.8$ $\mathrm{Hz}, 2 \mathrm{H}), 1.45(\mathrm{~d}, J=21.4 \mathrm{~Hz}, 6 \mathrm{H}) .{ }^{13} \mathrm{C}$ NMR $\left(101 \mathrm{MHz}, \mathrm{CDCl}_{3}\right) \delta 165.8,131.7,131.1,129.1,128.1,94.2$ $(\mathrm{d}, J=166.3 \mathrm{~Hz}), 61.2(\mathrm{~d}, J=5.9 \mathrm{~Hz}), 39.7(\mathrm{~d}, J=23.1 \mathrm{~Hz}), 27.1(\mathrm{~d}, J=24.6 \mathrm{~Hz}) ;{ }^{19} \mathrm{~F}$ NMR $(470 \mathrm{MHz}$, $\left.\mathrm{CDCl}_{3}\right) \delta-138.5$ (m, 1F). HRMS-ESI calcd for $\mathrm{C}_{12} \mathrm{H}_{18} \mathrm{BrFNO}_{2}\left[\mathrm{M}+\mathrm{NH}_{4}\right]^{+}$306.0499, found 306.0475.

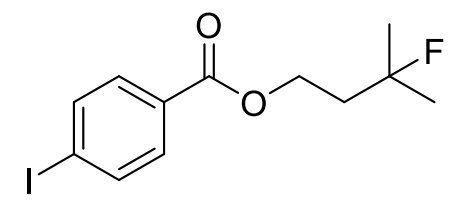

3-fluoro-3-methylbutyl 4-iodobenzoate (2h)

Following general procedure $\mathrm{C}$ using 3-methylbut-3-en-1-yl 4-iodobenzoate (1h, $63 \mathrm{mg}, 0.2 \mathrm{mmol}$ ) for 9 hours, analysis of the crude mixture indicated a 73\% NMR yield. The desired product was obtained as a colorless oil (49 $\mathrm{mg}, 0,15 \mathrm{mmol}, 73 \%)$ after purification by flash chromatography (toluene). Spectroscopic data agreed with the literature. ${ }^{3}{ }^{1} \mathrm{H}$ NMR $\left(500 \mathrm{MHz}, \mathrm{CDCl}_{3}\right) \delta 7.82-7.79(\mathrm{~m}, 2 \mathrm{H}), 7.75-7.72(\mathrm{~m}, 2 \mathrm{H}), 4.47(\mathrm{t}, J=6.8 \mathrm{~Hz}, 3 \mathrm{H}), 2.11(\mathrm{dt}, J=$ 19.6, $6.8 \mathrm{~Hz}, 3 \mathrm{H}), 1.44(\mathrm{~d}, J=21.4 \mathrm{~Hz}, 3 \mathrm{H}) ;{ }^{19} \mathrm{~F}$ NMR $\left(470 \mathrm{MHz}, \mathrm{CDCl}_{3}\right) \delta-138.4(\mathrm{~m}, 1 \mathrm{~F})$. 


\section{3-fluoro-3-methylbutyl 3,5-bis(trifluoromethyl)benzoate (2i)}

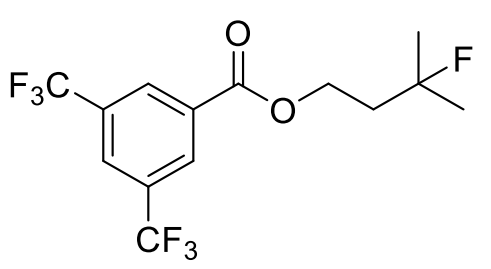

Following general procedure $\mathrm{C}$ using 3-methylbut-3-en-1-yl 3,5bis(trifluoromethyl)benzoate (1i, $65 \mathrm{mg}, 0.2 \mathrm{mmol}$ ) for 22 hours, analysis of the crude mixture indicated a 55\% NMR yield. The desired product was obtained as a colorless oil. (31 $\mathrm{mg}, 0.09 \mathrm{mmol}, 45 \%)$ after purification by flash chromatography (2.5\% $\mathrm{Et}_{2} \mathrm{O} /$ hexanes). IR (ATR, diamond), $v=2988,1732,1460,1377,1279,1256$, 1176, 1136, 912, $770 \mathrm{~cm}^{-1} ;{ }^{1} \mathrm{H}$ NMR $\left(500 \mathrm{MHz}, \mathrm{CDCl}_{3}\right) \delta 8.51-8.45(\mathrm{~m}, 2 \mathrm{H}), 8.09-8.05(\mathrm{~m}, 1 \mathrm{H}), 4.57$ $(\mathrm{t}, J=7.0 \mathrm{~Hz}, 2 \mathrm{H}), 2.16(\mathrm{dt}, J=19.9,7.0 \mathrm{~Hz}, 2 \mathrm{H}), 1.46(\mathrm{~d}, J=21.3 \mathrm{~Hz}, 6 \mathrm{H}) ;{ }^{13} \mathrm{C} \mathrm{NMR}\left(101 \mathrm{MHz}, \mathrm{CDCl}_{3}\right)$ $\delta 163.8,132.3,132.2(\mathrm{q}, J=34.1 \mathrm{~Hz}), 130.5-129.0(\mathrm{~m}), 126.5-126.2(\mathrm{~m}), 122.8(\mathrm{q}, J=272.8 \mathrm{~Hz}), 94.0$ $(\mathrm{d}, J=166.7 \mathrm{~Hz}), 62.1(\mathrm{~d}, J=5.7 \mathrm{~Hz}), 39.6(\mathrm{~d}, J=22.9 \mathrm{~Hz}), 27.0(\mathrm{~d}, J=24.5 \mathrm{~Hz}) ;{ }^{19} \mathrm{~F}$ NMR $(470 \mathrm{MHz}$, $\left.\mathrm{CDCl}_{3}\right) \delta-63.0$ (s, 6F), -139.5 (m, 1F). HRMS-ESI calcd for $\mathrm{C}_{14} \mathrm{H}_{17} \mathrm{~F}_{7} \mathrm{NO}_{2}\left[\mathrm{M}+\mathrm{NH}_{4}\right]^{+} 364.1142$, found 364.1135 .

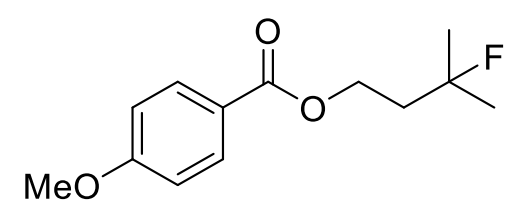

\section{3-fluoro-3-methylbutyl 4-methoxybenzoate (2j)}

Following general procedure $\mathrm{C}$ using 3-methylbut-3-en-1-yl 4methoxybenzoate $(\mathbf{1 j}, 44 \mathrm{mg}, 0.2 \mathrm{mmol})$ for 24 hours, analysis of the crude mixture indicated a 66\% NMR yield. The desired product was obtained as a colorless oil (26 mg, $0.11 \mathrm{mmol}, 53 \%)$ after purification by flash chromatography (40\% toluene/hexanes to $80 \%$ toluene/hexanes). Spectroscopic data agreed with the literature. ${ }^{1}{ }^{1} \mathrm{H}$ NMR $\left(500 \mathrm{MHz}, \mathrm{CDCl}_{3}\right) \delta 8.02-$ 7.96 (m, 2H), $6.96-6.88(\mathrm{~m}, 2 \mathrm{H}), 4.45$ (t, $J=6.8 \mathrm{~Hz}, 2 \mathrm{H}), 3.86(\mathrm{~s}, 3 \mathrm{H}), 2.11(\mathrm{dt}, J=19.4,6.8 \mathrm{~Hz}, 2 \mathrm{H})$, $1.45(\mathrm{~d}, J=21.5 \mathrm{~Hz}, 6 \mathrm{H}) ;{ }^{19} \mathrm{~F}$ NMR $\left(470 \mathrm{MHz}, \mathrm{CDCl}_{3}\right) \delta-137.9(\mathrm{~m}, 1 \mathrm{~F})$.

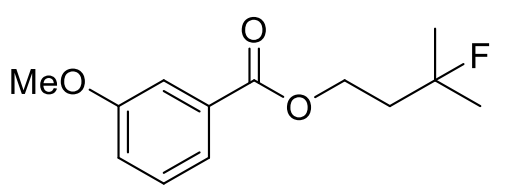

\section{3-fluoro-3-methylbutyl 3-methoxybenzoate (2k)}

Following general procedure $\mathrm{C}$ using 3-methylbut-3-en-1-yl 3methoxybenzoate (1k, $44 \mathrm{mg}, 0.2 \mathrm{mmol})$ for 4 hours, analysis of the crude

mixture indicated a 75\% NMR yield. The desired product was obtained as a colorless oil (29 mg, 0.12 $\mathrm{mmol}, 60 \%$ ) after purification by flash chromatography (80\% toluene/hexanes). Spectroscopic data agreed with the literature. ${ }^{1}{ }^{1} \mathrm{H}$ NMR $\left(500 \mathrm{MHz}, \mathrm{CDCl}_{3}\right) \delta 7.63(\mathrm{dt}, J=7.6,1.3 \mathrm{~Hz}, 1 \mathrm{H}), 7.56(\mathrm{dd}, J=2.7,1.5 \mathrm{~Hz}$, $1 \mathrm{H}), 7.35(\mathrm{t}, J=7.9 \mathrm{~Hz}, 1 \mathrm{H}), 7.10(\mathrm{dd}, J=7.8,2.1 \mathrm{~Hz}, 1 \mathrm{H}), 4.48(\mathrm{t}, J=6.8 \mathrm{~Hz}, 2 \mathrm{H}), 3.85(\mathrm{~s}, 3 \mathrm{H}), 2.12$ $(\mathrm{dt}, J=19.4,6.8 \mathrm{~Hz}, 2 \mathrm{H}), 1.45(\mathrm{~d}, J=21.5 \mathrm{~Hz}, 6 \mathrm{H}) ;{ }^{19} \mathrm{~F} \mathrm{NMR}\left(470 \mathrm{MHz}, \mathrm{CDCl}_{3}\right) \delta-138.2(\mathrm{~m}, 1 \mathrm{~F})$. 


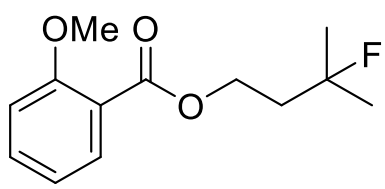

\section{3-fluoro-3-methylbutyl 2-methoxybenzoate (21)}

Following general procedure $\mathrm{C}$ using 3-methylbut-3-en-1-yl 2-methoxybenzoate

(11, $44 \mathrm{mg}, 0.2 \mathrm{mmol}$ ) the desired product was obtained after 7 hours (55\% NMR Yield; estimated by ${ }^{19} \mathrm{~F}$ NMR analysis of the crude mixture after workup using 2-fluoro-4-nitrotoluene as the internal standard). ${ }^{19} \mathrm{~F}$ NMR $\left(470 \mathrm{MHz}, \mathrm{CDCl}_{3}\right) \delta-137.7(\mathrm{~m}, 1 \mathrm{~F}){ }^{1}$

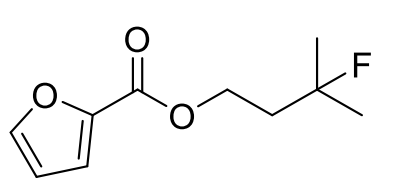

\section{3-fluoro-3-methylbutyl furan-2-carboxylate (2m)}

Following general procedure $\mathrm{C}$ using 3-methylbut-3-en-1-yl furan-2-carboxylate (1m, $36 \mathrm{mg}, 0.2 \mathrm{mmol}$ ) for 30 hours, analysis of the crude mixture indicated a 56\% NMR yield. The desired product was obtained as a colorless oil. (21 mg, $0.11 \mathrm{mmol}, 53 \%)$ after purification by flash chromatography (50\% toluene/hexanes). IR (ATR, diamond), $v=2984,2924,1728$, 1582, 1474, 1375, 1296, 1180, 1119, 1014, $762 \mathrm{~cm}^{-1}$; ${ }^{1} \mathrm{H}$ NMR (500 MHz, $\left.\mathrm{CDCl}_{3}\right) \delta 7.58$ (s, 1H), 7.17 (d, $J=3.5 \mathrm{~Hz}, 1 \mathrm{H}), 6.51(\mathrm{dd}, J=3.5,1.8 \mathrm{~Hz}, 1 \mathrm{H}), 4.46(\mathrm{t}, J=6.9 \mathrm{~Hz}, 2 \mathrm{H}), 2.10(\mathrm{dt}, J=19.4,6.9 \mathrm{~Hz}, 2 \mathrm{H})$, $1.43(\mathrm{~d}, J=21.4 \mathrm{~Hz}, 6 \mathrm{H}) ;{ }^{13} \mathrm{C} \mathrm{NMR}\left(126 \mathrm{MHz}, \mathrm{CDCl}_{3}\right) \delta 158.6,146.4,144.6,117.9,111.8,94.2(\mathrm{~d}, J=$ $166.1 \mathrm{~Hz}), 60.9(\mathrm{~d}, J=6.4 \mathrm{~Hz}), 39.8(\mathrm{~d}, J=23.3 \mathrm{~Hz}), 27.0(\mathrm{~d}, J=24.4 \mathrm{~Hz}) ;{ }^{19} \mathrm{~F}$ NMR $\left(470 \mathrm{MHz}, \mathrm{CDCl}_{3}\right)$ $\delta$-138.4 (m, 1F); HRMS-ESI calcd for $\mathrm{C}_{10} \mathrm{H}_{14} \mathrm{FO}_{3}[\mathrm{M}+\mathrm{H}]^{+} 201.0921$ found 201.0907.

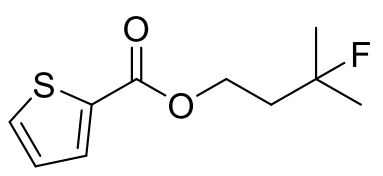

\section{3-fluoro-3-methylbutyl thiophene-2-carboxylate (2n)}

Following general procedure $\mathrm{C}$ using 3-methylbut-3-en-1-yl thiophene-2carboxylate (1n, $39 \mathrm{mg}, 0.2 \mathrm{mmol}$ ) for 24 hours, analysis of the crude mixture indicated a 56\% NMR yield. The desired product was obtained as a colorless oil. ( $25 \mathrm{mg}, 0.12 \mathrm{mmol}$, $57 \%$ ) after purification by flash chromatography (50\% toluene/hexanes). Spectroscopic data agreed with the literature. ${ }^{6}{ }^{1} \mathrm{H}$ NMR $\left(500 \mathrm{MHz}, \mathrm{CDCl}_{3}\right) \delta 7.80(\mathrm{dd}, J=3.7,1.3 \mathrm{~Hz}, 1 \mathrm{H}), 7.56(\mathrm{dd}, J=5.0,1.3 \mathrm{~Hz}, 1 \mathrm{H})$, $7.10(\mathrm{dd}, J=5.0,3.7 \mathrm{~Hz}, 1 \mathrm{H}), 4.45(\mathrm{t}, J=6.8 \mathrm{~Hz}, 2 \mathrm{H}), 2.10(\mathrm{dt}, J=19.3,6.8 \mathrm{~Hz}, 2 \mathrm{H}), 1.45(\mathrm{~d}, J=21.5$ $\mathrm{Hz}, 6 \mathrm{H}) ;{ }^{19} \mathrm{~F} \mathrm{NMR}\left(470 \mathrm{MHz}, \mathrm{CDCl}_{3}\right) \delta-138.2(\mathrm{~m}, 1 \mathrm{~F})$.

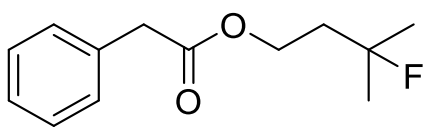

\section{3-fluoro-3-methylbutyl 2-phenylacetate (2o)}

Following general procedure $\mathrm{C}$ using 3-methylbut-3-en-1-yl 2-phenylacetate (1o, $41 \mathrm{mg}, 0.2 \mathrm{mmol}$ ) for 10 hours, analysis of the crude mixture indicated a 65\% NMR yield. The desired product was obtained as a colorless oil. (28 $\mathrm{mg}, 0.13 \mathrm{mmol}, 63 \%)$ after purification by flash chromatography (50\% toluene/hexanes). IR (ATR, diamond), $v=2982,2928,1734,1497,1454,1387$, 
1248, 1148, 1009, $860 \mathrm{~cm}^{-1} ;{ }^{1} \mathrm{H}$ NMR (400 MHz, $\left.\mathrm{CDCl}_{3}\right) \delta 7.36-7.23(\mathrm{~m}, 5 \mathrm{H}), 4.24(\mathrm{t}, J=6.8 \mathrm{~Hz}, 2 \mathrm{H})$, $3.61(\mathrm{~s}, 0 \mathrm{H}), 1.95(\mathrm{dt}, J=19.4,6.9 \mathrm{~Hz}, 1 \mathrm{H}), 1.33(\mathrm{~d}, J=21.5 \mathrm{~Hz}, 1 \mathrm{H}) ;{ }^{13} \mathrm{C} \mathrm{NMR}\left(101 \mathrm{MHz}, \mathrm{CDCl}_{3}\right) \delta$ 171.5, 133.9, 129.3, 128.6, 127.1, $94.2(\mathrm{~d}, J=165.8 \mathrm{~Hz}), 60.8(\mathrm{~d}, J=6.3 \mathrm{~Hz}), 41.5,39.6(\mathrm{~d}, J=23.1 \mathrm{~Hz})$, $26.9(\mathrm{~d}, J=24.6 \mathrm{~Hz}) ;{ }^{19} \mathrm{~F} \mathrm{NMR}\left(470 \mathrm{MHz}, \mathrm{CDCl}_{3}\right) \delta-138.3(\mathrm{~m}, 1 \mathrm{~F})$. HRMS-ESI calcd for $\mathrm{C}_{13} \mathrm{H}_{21} \mathrm{FNO}_{2}$ $\left[\mathrm{M}+\mathrm{NH}_{4}\right]^{+} 242.1545$, found 242.1551 .

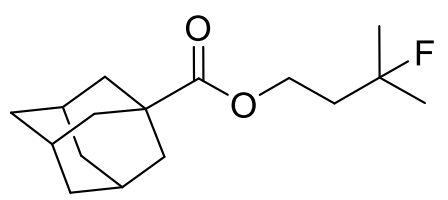

\section{3-fluoro-3-methylbutyl $(3 r, 5 r, 7 r)$-adamantane-1-carboxylate (2p)}

Following general procedure $\mathrm{C}$ using 3-methylbut-3-en-1-yl $(3 r, 5 r, 7 r)$ adamantane-1-carboxylate $(\mathbf{1 p}, 50 \mathrm{mg}, 0.2 \mathrm{mmol})$ for 30 hours, analysis of the crude mixture indicated a 61\% NMR yield. The desired product was obtained as a colorless oil. (23 $\mathrm{mg}, 0.08 \mathrm{mmol}, 42 \%)$ after purification by flash chromatography (50\% toluene/hexanes). IR (ATR, diamond), $v=2907,2853,1726,1454,1373,1267,1227,1103,1078,862 \mathrm{~cm}^{-1} ;{ }^{1} \mathrm{H}$ NMR $(500 \mathrm{MHz}$, $\left.\mathrm{CDCl}_{3}\right) \delta 4.20(\mathrm{t}, J=6.7 \mathrm{~Hz}, 2 \mathrm{H}), 2.03-1.93(\mathrm{~m}, 5 \mathrm{H}), 1.88(\mathrm{~d}, J=2.8 \mathrm{~Hz}, 6 \mathrm{H}), 1.75-1.67(\mathrm{~m}, 6 \mathrm{H}), 1.40$ $(\mathrm{d}, J=21.5 \mathrm{~Hz}, 6 \mathrm{H}) .{ }^{13} \mathrm{C} \mathrm{NMR}\left(101 \mathrm{MHz}, \mathrm{CDCl}_{3}\right) \delta 177.6,94.3(\mathrm{~d}, J=165.7 \mathrm{~Hz}), 60.1(\mathrm{~d}, J=6.3 \mathrm{~Hz})$, 40.6, $39.8(\mathrm{~d}, J=23.3 \mathrm{~Hz}), 38.8,36.5,27.9,27.1(\mathrm{~d}, J=24.6 \mathrm{~Hz}) ;{ }^{19} \mathrm{~F} \mathrm{NMR}\left(470 \mathrm{MHz}, \mathrm{CDCl}_{3}\right) \delta-137.8$ (m, 1F). HRMS-ESI calcd for $\mathrm{C}_{16} \mathrm{H}_{29} \mathrm{FNO}_{2}\left[\mathrm{M}+\mathrm{NH}_{4}\right]^{+}$286.2177, found 286.2183.

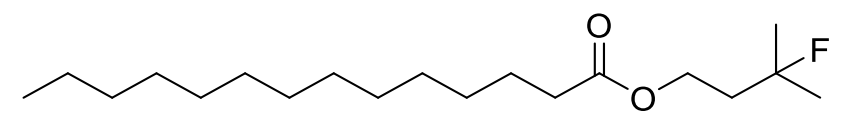

\section{3-fluoro-3-methylbutyltetradecanoate (2q)}

Following general procedure C using 3-methylbut-3en-1-yl tetradecanoate (1q, $59 \mathrm{mg}, 0.2 \mathrm{mmol})$ for 24 hours, analysis of the crude mixture indicated a $60 \%$ NMR yield. The desired product was obtained as a colorless oil. (39 mg, $0.12 \mathrm{mmol}, 62 \%$ ) after purification by flash chromatography ( $25 \%$ toluene/hexanes to $100 \%$ toluene). IR (ATR, diamond), $v=$ 2957, 2926, 2854, 1738, 1466, 1375, 1271, 1171, 908, $735 \mathrm{~cm}^{-1} ;{ }^{1} \mathrm{H}$ NMR $\left(500 \mathrm{MHz}, \mathrm{CDCl}_{3}\right) \delta 4.22(\mathrm{t}, J$ $=7.0 \mathrm{~Hz}, 2 \mathrm{H}), 2.29(\mathrm{t}, J=7.5 \mathrm{~Hz}, 2 \mathrm{H}), 1.98(\mathrm{dt}, J=19.5,6.9 \mathrm{~Hz}, 2 \mathrm{H}), 1.61(\mathrm{~m}, 2 \mathrm{H}), 1.39(\mathrm{~d}, J=21.5 \mathrm{~Hz}$, $6 \mathrm{H}), 1.26(\mathrm{~m}, 20 \mathrm{H}), 0.88(\mathrm{t}, J=7.0 \mathrm{~Hz}, 3 \mathrm{H}) ;{ }^{13} \mathrm{C} \mathrm{NMR}\left(126 \mathrm{MHz}, \mathrm{CDCl}_{3}\right) \delta 173.8,94.2(\mathrm{~d}, J=165.9 \mathrm{~Hz})$, $60.2(\mathrm{~d}, J=6.5 \mathrm{~Hz}), 39.8(\mathrm{~d}, J=23.3 \mathrm{~Hz}), 34.4,31.9,29.7,29.64(2 \mathrm{C}), 29.59,29.5,29.4,29.3,29.2,27.0$ $(\mathrm{d}, J=24.5 \mathrm{~Hz}), 24.9,22.7,14.12 ;{ }^{19} \mathrm{~F} \mathrm{NMR}\left(470 \mathrm{MHz}, \mathrm{CDCl}_{3}\right) \delta-138.2(\mathrm{~m}, 1 \mathrm{~F})$; HRMS-ESI calcd for $\mathrm{C}_{19} \mathrm{H}_{41} \mathrm{FNO}_{2}\left[\mathrm{M}+\mathrm{NH}_{4}\right]^{+}$334.3116, found 334.3105. 


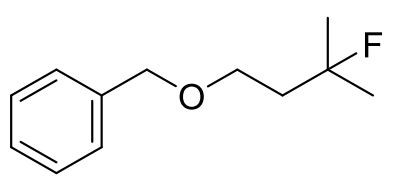

((3-fluoro-3-methylbutoxy)methyl)benzene (2r)

Following general procedure $\mathrm{C}$ using (((3-methylbut-3-en-1yl)oxy)methyl)benzene (44 $\mathrm{mg}, 0.2 \mathrm{mmol})$ the desired product was obtained after 4 hours (68\% NMR Yield; estimated by ${ }^{19} \mathrm{~F}$ NMR analysis of the crude mixture after workup using 2-fluoro-4-nitrotoluene as the internal standard). ${ }^{19} \mathrm{~F} \mathrm{NMR}\left(470 \mathrm{MHz}, \mathrm{CDCl}_{3}\right) \delta-136.5$ (m, 1F)

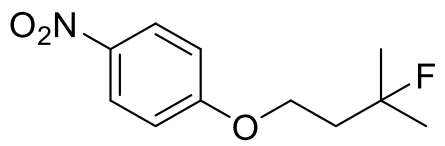

\section{1-(3-fluoro-3-methylbutoxy)-4-nitrobenzene (2s)}

Following general procedure $\mathrm{C}$ using 1-((3-methylbut-3-en-1-yl)oxy)-4nitrobenzene $(1 \mathrm{~s}, 42 \mathrm{mg}, 0.2 \mathrm{mmol})$ for 30 hours, analysis of the crude mixture indicated a 67\% NMR yield. The desired product was obtained as a colorless oil (29 mg, 0.13 $\mathrm{mmol}, 64 \%$ ) after purification by flash chromatography (75\% toluene/hexanes). Spectroscopic data agreed with the literature. ${ }^{6}{ }^{1} \mathrm{H}$ NMR $\left(500 \mathrm{MHz}, \mathrm{CDCl}_{3}\right) \delta 8.23-8.19(\mathrm{~m}, 2 \mathrm{H}), 6.98-6.95(\mathrm{~m}, 2 \mathrm{H}), 4.24(\mathrm{t}, J=$ $6.7 \mathrm{~Hz}, 2 \mathrm{H}), 2.18(\mathrm{dt}, J=19.9,6.7 \mathrm{~Hz}, 2 \mathrm{H}), 1.46(\mathrm{~d}, J=21.5 \mathrm{~Hz}, 6 \mathrm{H}) ;{ }^{19} \mathrm{~F}$ NMR $\left(470 \mathrm{MHz}, \mathrm{CDCl}_{3}\right) \delta-$ $138.9(\mathrm{~m}, 1 \mathrm{~F})$.

\section{$L_{F}$ 3-fluoro-3-methylbutyl 4-methylbenzenesulfonate (2t)}

TsO Following general procedure $\mathrm{C}$ using 3-methylbut-3-en-1-yl 4-methylbenzenesulfonate (1t, $48 \mathrm{mg}, 0.2 \mathrm{mmol}$ ) for 72 hours, analysis of the crude mixture indicated a 65\% NMR yield. The desired product was obtained as a colorless oil. (28 $\mathrm{mg}, 0.11 \mathrm{mmol}, 54 \%)$ after purification by flash chromatography (100\% toluene). Spectroscopic data agreed with the literature. ${ }^{1}{ }^{1} \mathrm{H}$ NMR $(500 \mathrm{MHz}$, $\left.\mathrm{CDCl}_{3}\right) \delta 7.80(\mathrm{~d}, J=8.0 \mathrm{~Hz}, 2 \mathrm{H}), 7.36(\mathrm{~d}, J=8.0 \mathrm{~Hz}, 2 \mathrm{H}), 4.17(\mathrm{t}, J=6.8 \mathrm{~Hz}, 2 \mathrm{H}), 2.46(\mathrm{~s}, 3 \mathrm{H}), 2.01$ $(\mathrm{dt}, J=19.6,6.8 \mathrm{~Hz}, 2 \mathrm{H}), 1.34(\mathrm{~d}, J=21.4 \mathrm{~Hz}, 6 \mathrm{H}) ;{ }^{19} \mathrm{~F} \mathrm{NMR}\left(470 \mathrm{MHz}, \mathrm{CDCl}_{3}\right) \delta-139.1(\mathrm{~m}, 1 \mathrm{~F})$.

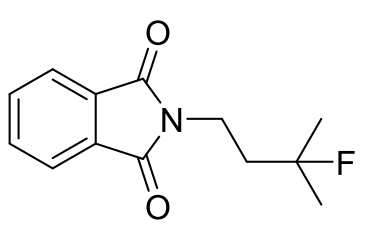

\section{2-(3-fluoro-3-methylbutyl)isoindoline-1,3-dione (2u)}

Following general procedure $\mathrm{C}$ using 2-(3-methylbut-3-en-1-yl)isoindoline-1,3dione (1u, $48 \mathrm{mg}, 0.2 \mathrm{mmol})$ for 96 hours, analysis of the crude mixture indicated a 43\% NMR yield. The desired product was obtained as a colorless oil. (19 mg, $0.07 \mathrm{mmol}, 37 \%)$ after purification by flash chromatography (100\% toluene). Spectroscopic data agreed with the literature. ${ }^{16}{ }^{1} \mathrm{H}$ NMR $\left(500 \mathrm{MHz}, \mathrm{CDCl}_{3}\right) \delta 7.87-7.81(\mathrm{~m}, 2 \mathrm{H}), 7.75-7.68(\mathrm{~m}, 2 \mathrm{H}), 3.89-3.81$ $(\mathrm{m}, 2 \mathrm{H}), 2.02(\mathrm{dt}, J=19.9,7.8 \mathrm{~Hz}, 2 \mathrm{H}), 1.44(\mathrm{~d}, J=21.4 \mathrm{~Hz}, 6 \mathrm{H}) ;{ }^{19} \mathrm{~F} \mathrm{NMR}\left(470 \mathrm{MHz}, \mathrm{CDCl}_{3}\right) \delta-141.1$ $(\mathrm{m}, 1 \mathrm{~F})$. 
$\mathrm{C}_{\mathrm{BzO}}^{\mathrm{F}}$

\section{(S)-7-fluoro-3,7-dimethyloctyl benzoate (2v)}

Following general procedure $\mathrm{C}$ using (S)-3,7-dimethyloct-6-en-1-yl benzoate (1v, $52 \mathrm{mg}, 0.2 \mathrm{mmol}$ ) for 30 hours, analysis of the crude mixture indicated a 58\% NMR yield. The desired product was obtained as a colorless oil. (30 $\mathrm{mg}, 54 \%)$ after purification by flash chromatography (50\% toluene/hexanes). $[\alpha]_{D}^{20}=-2.0$ (c 1.0, $\mathrm{CHCl}_{3}$ ); IR (ATR, diamond), $v=2943,2874,1718,1452,1385$, 1315, 1273, 1113, 1026, $712 \mathrm{~cm}^{-1}$; ${ }^{1} \mathrm{H}$ NMR (500 MHz, $\left.\mathrm{CDCl}_{3}\right) \delta 8.07-8.02(\mathrm{~m}, 2 \mathrm{H}), 7.58-7.53(\mathrm{~m}$, 1H), $7.47-7.41(\mathrm{~m}, 2 \mathrm{H}), 4.41-4.32(\mathrm{~m}, 2 \mathrm{H}), 1.86-1.79(\mathrm{~m}, 1 \mathrm{H}), 1.70-1.64(\mathrm{~m}, 1 \mathrm{H}), 1.62-1.55(\mathrm{~m}$, $3 \mathrm{H}), 1.49-1.37(\mathrm{~m}, 3 \mathrm{H}), 1.33(\mathrm{~d}, J=21.2 \mathrm{~Hz}, 6 \mathrm{H}), 1.25-1.20(\mathrm{~m}, 1 \mathrm{H}), 0.97(\mathrm{~d}, J=6.6 \mathrm{~Hz}, 3 \mathrm{H}) ;{ }^{13} \mathrm{C}$ NMR $\left(101 \mathrm{MHz}, \mathrm{CDCl}_{3}\right) \delta 166.7,132.8,130.5,129.5,128.3,95.7$ (d, $\left.J=164.5 \mathrm{~Hz}\right), 63.5,41.6(\mathrm{~d}, J=$ $22.7 \mathrm{~Hz}), 37.2,35.5,29.9,26.7(\mathrm{~d}, J=24.9 \mathrm{~Hz}), 26.6(\mathrm{~d}, J=24.9 \mathrm{~Hz}), 21.3(\mathrm{~d}, J=5.2 \mathrm{~Hz}), 19.5 ;{ }^{19} \mathrm{~F} \mathrm{NMR}$ $\left(470 \mathrm{MHz}, \mathrm{CDCl}_{3}\right) \delta-137.5$ (m, 1F). HRMS-ESI calcd for $\mathrm{C}_{17} \mathrm{H}_{29} \mathrm{FNO}_{2}\left[\mathrm{M}+\mathrm{NH}_{4}\right]^{+}$298.2177, found 298.2175 .

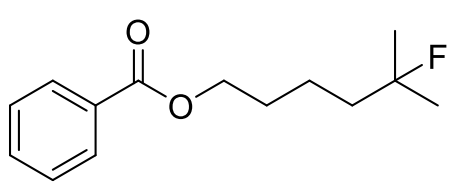

\section{5-fluoro-5-methylhexyl benzoate (2w).}

Following general procedure $\mathrm{C}$ using 5-methylhex-5-en-1-yl benzoate (1w, $44 \mathrm{mg}, 0.2 \mathrm{mmol}$ ) for 24 hours, analysis of the crude mixture indicated a $74 \%$ NMR yield. The desired product was obtained as a colorless oil (23 mg, $0.1 \mathrm{mmol}, 49 \%)$ after purification by flash chromatography (100\% toluene). IR (ATR, diamond), $v=2980,2949,1717,1585$, 1452, 1373, 1313, 1271, 1113, $1070 \mathrm{~cm}^{-1} ;{ }^{1} \mathrm{H}$ NMR $\left(400 \mathrm{MHz}, \mathrm{CDCl}_{3}\right) \delta 8.10-7.99(\mathrm{~m}, 2 \mathrm{H}), 7.59-7.52$ (m, 1H), $7.44(\mathrm{t}, J=7.7 \mathrm{~Hz}, 2 \mathrm{H}), 4.34(\mathrm{t}, J=6.6 \mathrm{~Hz}, 2 \mathrm{H}), 1.80(\mathrm{p}, J=6.9 \mathrm{~Hz}, 2 \mathrm{H}), 1.73-1.63(\mathrm{~m}, 2 \mathrm{H})$, $1.61-1.53(\mathrm{~m}, 2 \mathrm{H}), 1.36(\mathrm{~d}, J=21.4 \mathrm{~Hz}, 6 \mathrm{H}) ;{ }^{13} \mathrm{C} \mathrm{NMR}\left(126 \mathrm{MHz}, \mathrm{CDCl}_{3}\right) \delta 166.6,132.9,130.4,129.5$, 128.3, $95.5(\mathrm{~d}, J=164.9 \mathrm{~Hz}), 64.8,41.0(\mathrm{~d}, J=22.9 \mathrm{~Hz}), 29,26.6(\mathrm{~d}, J=24.8 \mathrm{~Hz}), 20.5(\mathrm{~d}, J=5.2 \mathrm{~Hz})$; ${ }^{19} \mathrm{~F}$ NMR $\left(470 \mathrm{MHz}, \mathrm{CDCl}_{3}\right) \delta-138.2(\mathrm{~m}, 1 \mathrm{~F})$; HRMS-ESI calcd for $\mathrm{C}_{14} \mathrm{H}_{23} \mathrm{FNO}_{2}\left[\mathrm{M}+\mathrm{NH}_{4}\right]^{+} 256.1707$, found 256.1698

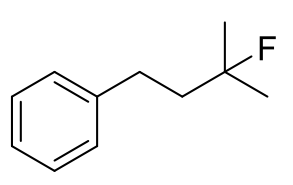

\section{(3-fluoro-3-methylbutyl)benzene (2x).}

Following general procedure $\mathrm{C}$ using (3-methylbut-3-en-1-yl)benzene (1x, $29 \mathrm{mg}, 0.2$ mmol) for 20 hours, analysis of the crude mixture indicated a 68\% NMR yield. The desired product was obtained as a colorless oil $(20 \mathrm{mg}, 0.12 \mathrm{mmol}, 59 \%)$ after purification by flash chromatography ( $\left.2 \% \mathrm{Et}_{2} \mathrm{O} / \mathrm{Hexanes}\right)$. Spectroscopic data agreed with the literature. ${ }^{17}{ }^{1} \mathrm{H} \mathrm{NMR}(400 \mathrm{MHz}$,

\footnotetext{
${ }^{17}$ Dryzhakov, M.; Moran, J. ACS Catal. 2016, 6, 3670-3673
} 
$\left.\mathrm{CDCl}_{3}\right) \delta 7.34-7.25(\mathrm{~m}, 2 \mathrm{H}), 7.24-7.17(\mathrm{~m}, 3 \mathrm{H}), 2.77-2.68(\mathrm{~m}, 2 \mathrm{H}), 1.99-1.85(\mathrm{~m}, 2 \mathrm{H}), 1.41(\mathrm{~d}, J=$ $21.4 \mathrm{~Hz}, 6 \mathrm{H}) ;{ }^{19} \mathrm{~F}$ NMR $\left(470 \mathrm{MHz}, \mathrm{CDCl}_{3}\right) \delta-138.9(\mathrm{~m}, 1 \mathrm{~F})$.<smiles>CC(C)(F)CCOC(=O)c1cccnc1</smiles>

\section{3-methylbut-3-en-1-yl nicotinate (2y)}

Following general procedure $\mathrm{C}$ using 3-methylbut-3-en-1-yl nicotinate (1w, 38 $\mathrm{mg}, 0.2 \mathrm{mmol})$ the desired product was obtained after 4 hours (5\% NMR Yield; estimated by ${ }^{19} \mathrm{~F}$ NMR analysis of the crude mixture after workup using 2-fluoro-4-nitrotoluene as the internal standard). ${ }^{19} \mathrm{~F} \mathrm{NMR}\left(470 \mathrm{MHz}, \mathrm{CDCl}_{3}\right) \delta-138.8(\mathrm{~m}, 1 \mathrm{~F}){ }^{14}$

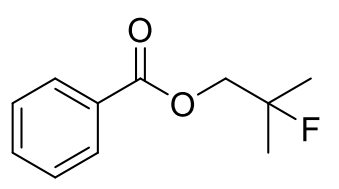

\section{2-fluoro-2-methylpropyl benzoate (2z).}

Following general procedure $\mathrm{C}$ using (((3-methylbut-3-en-1-yl)oxy)methyl)benzene (1x, $35 \mathrm{mg}, 0.2 \mathrm{mmol})$ the desired product was obtained after 4 days (4\% NMR Yield; estimated by ${ }^{19} \mathrm{~F}$ NMR analysis of the crude mixture after workup using 2-fluoro-4-nitrotoluene as the internal standard). ${ }^{19} \mathrm{~F}$ NMR $\left(470 \mathrm{MHz}, \mathrm{CDCl}_{3}\right) \delta-145.8(\mathrm{~m}, 1 \mathrm{~F}){ }^{18}$

\section{Continuous flow reaction}

3-fluoro-3-methylbutyl benzoate (2a)
A solution of 3-methylbut-3-en-1-yl benzoate $(38 \mathrm{mg}, 0.2 \mathrm{mmol})$ and triethylamine trihydrofluoride (163 $\mu \mathrm{L}, 1 \mathrm{mmol}, 5.0$ equiv.) in $0.5 \mathrm{~mL}$ of $\mathrm{CH}_{2} \mathrm{Cl}_{2}$ and a solution of methanesulfonic acid (65 $\mu \mathrm{L}, 1 \mathrm{mmol}, 5.0$ equiv.) in $0.5 \mathrm{~mL}$ of $\mathrm{CH}_{2} \mathrm{Cl}_{2}$ were injected through a PTFE reactor $(5 \times 10 \mathrm{~mL})$ at a $1.5 \mathrm{~mL} / \mathrm{min}$ each for a flow rate of $3 \mathrm{~mL} / \mathrm{min}$ and a residence time of 16.67 minutes. The reaction mixture was collected in a round bottom flask containing a saturated aqueous solution of $\mathrm{NaHCO}_{3}$. The aqueous phase was extracted with $\mathrm{CH}_{2} \mathrm{Cl}_{2}(3 \mathrm{x})$, dried over $\mathrm{Na}_{2} \mathrm{SO}_{4}$, filtered and concentrated under reduced pressure. (36\% NMR Yield; estimated by ${ }^{19} \mathrm{~F}$ NMR analysis of the crude mixture after workup using 2fluoro-4-nitrotoluene as the internal standard). ${ }^{19} \mathrm{~F}$ NMR $\left(470 \mathrm{MHz}, \mathrm{CDCl}_{3}\right) \delta-138.2(\mathrm{~m}, 1 \mathrm{~F})$

\section{NMR Spectra}

\footnotetext{
${ }^{18}$ Zhang, X.; Guo, S.; Tang, P. Org. Chem. Front. 2015, 2, 806-810.
} 


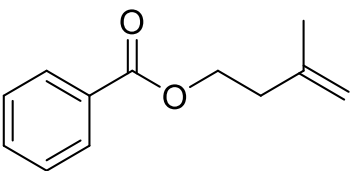

$1 a$

${ }^{1} \mathrm{H}$ NMR, $500 \mathrm{MHz}, \mathrm{CDCl}_{3}$

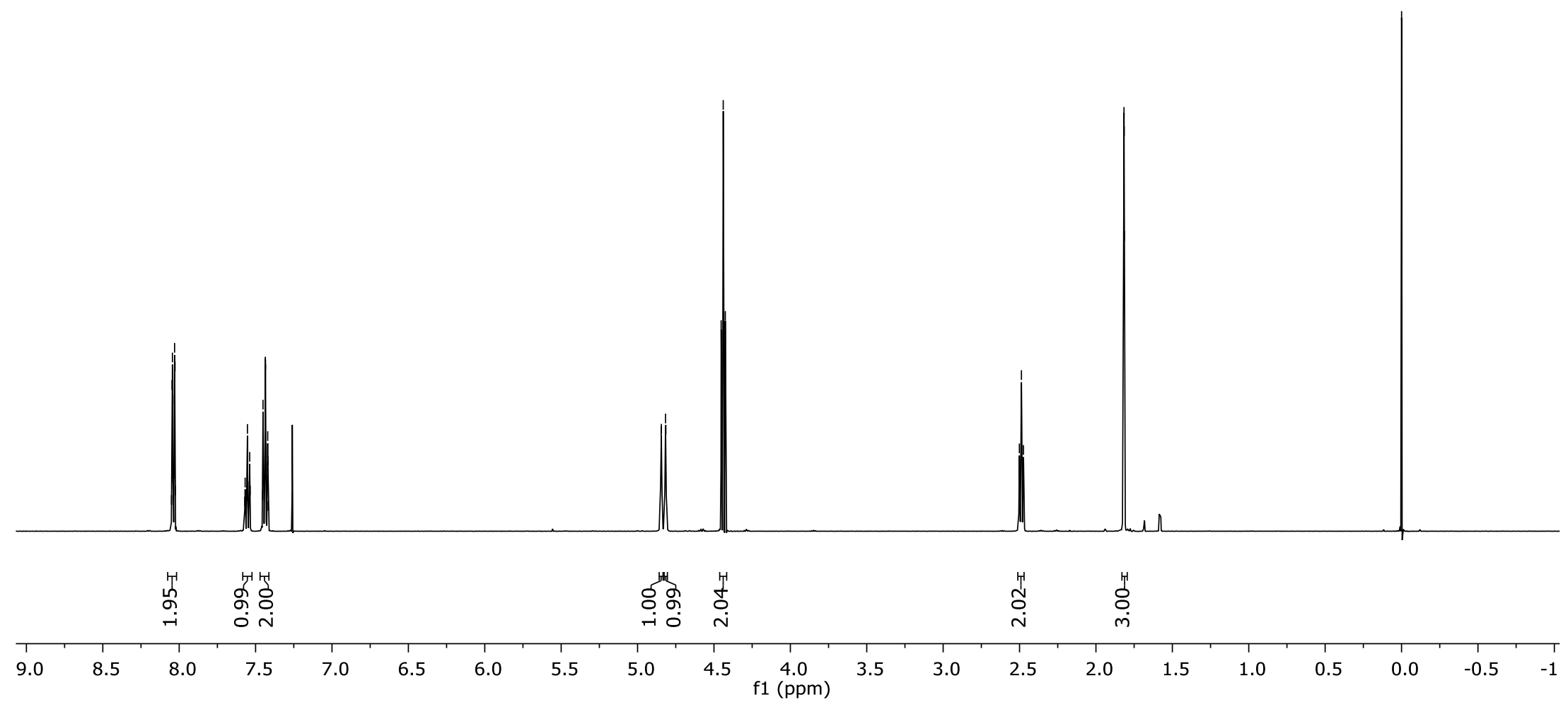




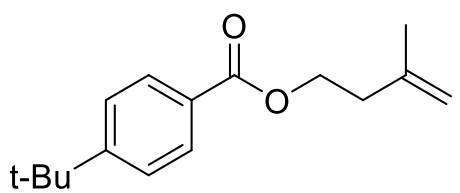

$1 \mathrm{~b}$

${ }^{1} \mathrm{H}$ NMR, $400 \mathrm{MHz}^{\mathrm{CDCl}}{ }_{3}$

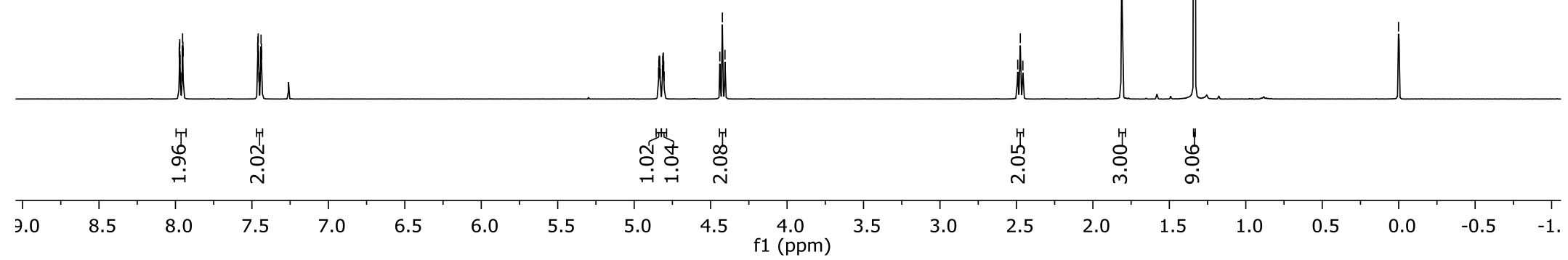


<smiles>C=C(C)CCOC(=O)c1ccc(-c2ccccc2)cc1</smiles>

$1 \mathrm{c}$

${ }^{1} \mathrm{H}$ NMR, $400 \mathrm{MHz}, \mathrm{CDCl}_{3}$

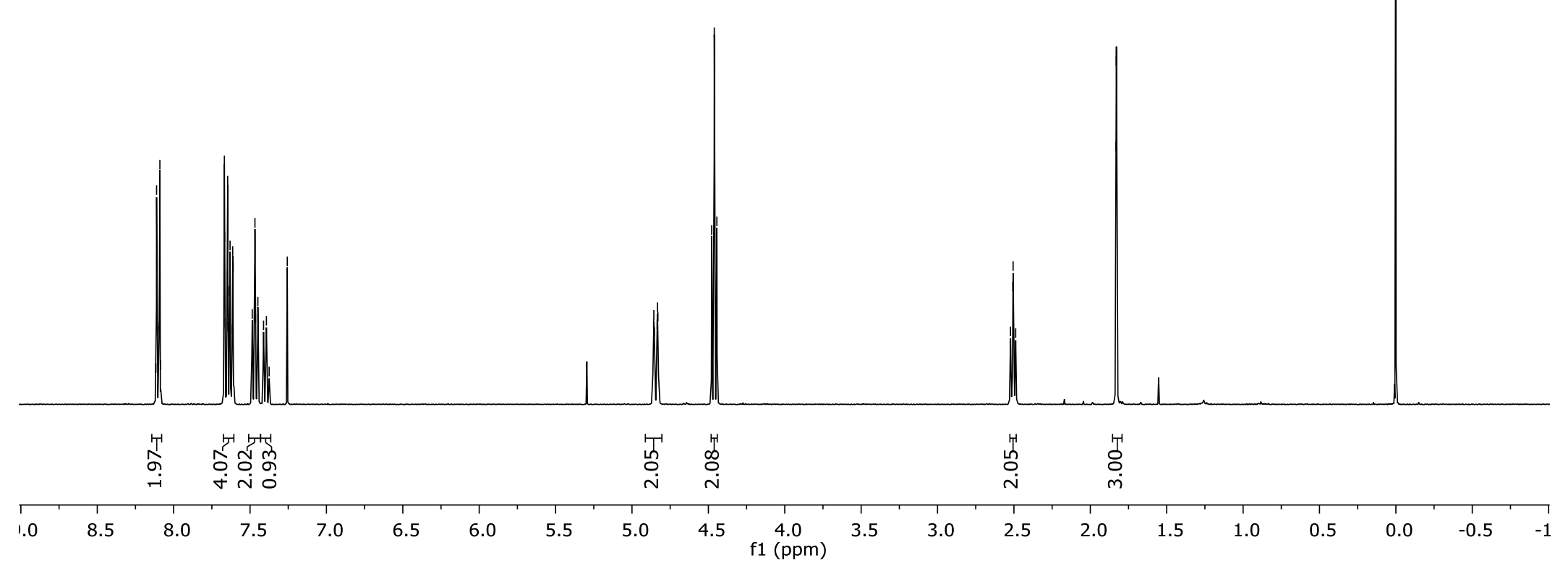




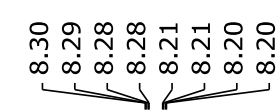

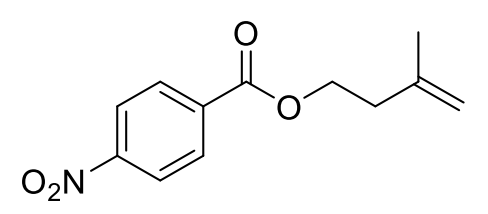

$1 d$

${ }^{1} \mathrm{H}$ NMR, $500 \mathrm{MHz}, \mathrm{CDCl}_{3}$
ํํㄴ ํํำ

Nก

$\sqrt{1.4}$

$\stackrel{\infty}{\stackrel{\infty}{\perp}}$

:

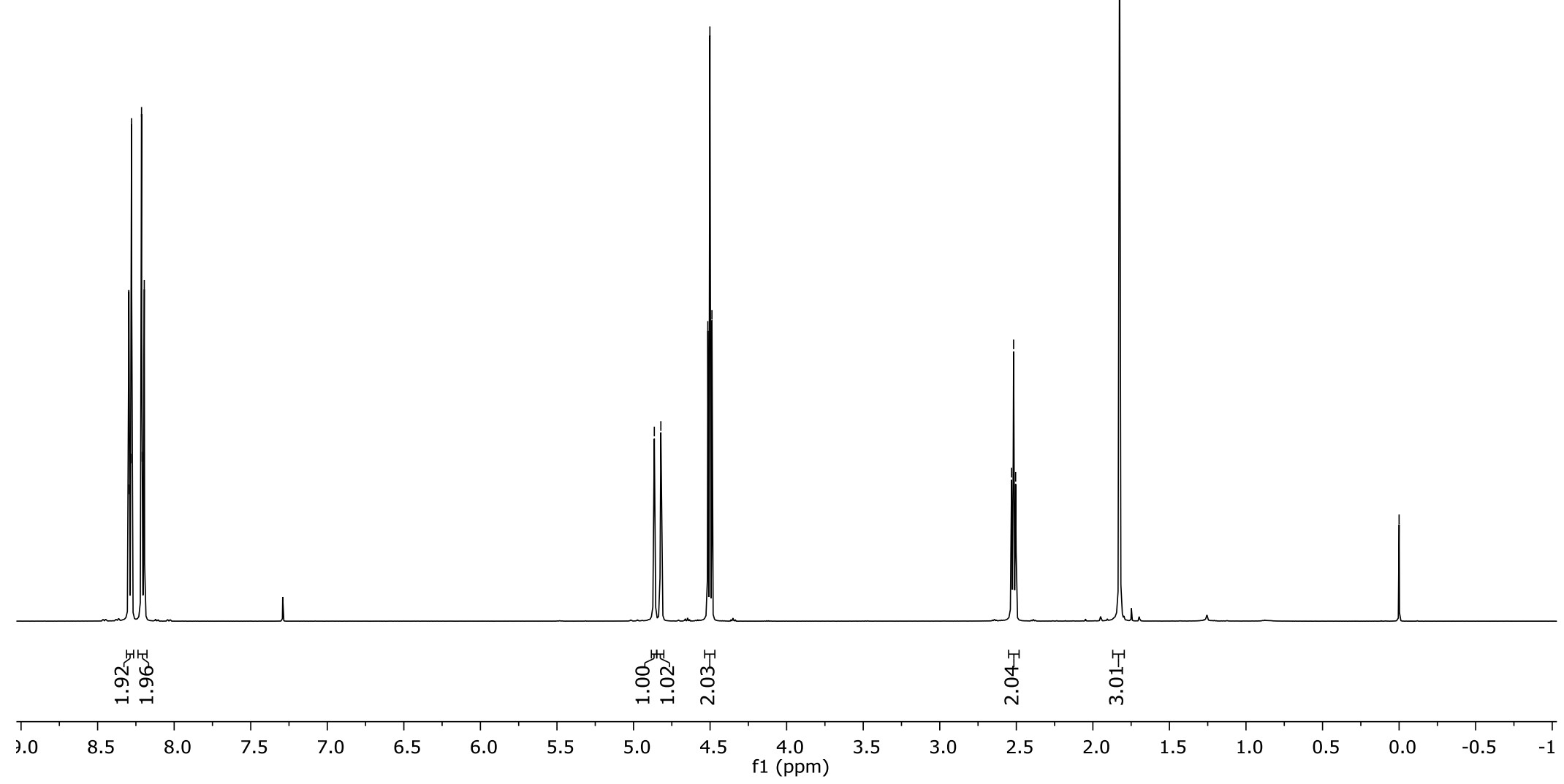




\begin{tabular}{|c|c|c|c|c|c|}
\hline $\begin{array}{l}\vec{n} \\
0 \\
0 \\
\text { h } \\
\text { | }\end{array}$ & 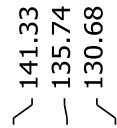 & \begin{tabular}{c}
\multirow{H}{*}{} \\
$\stackrel{\sim}{\sim}$ \\
\multirow{1}{*}{}
\end{tabular} & 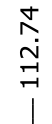 & $\begin{array}{l}\text { जे } \\
\text { రु } \\
\mid\end{array}$ & $\begin{array}{l}\hat{N} \\
\dot{m} \\
\dot{m}\end{array}$ \\
\hline
\end{tabular}

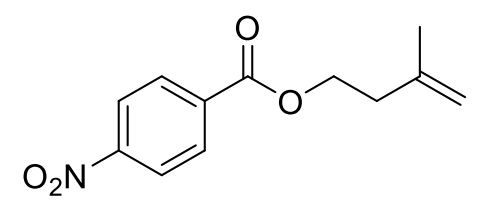

$1 d$

${ }^{13} \mathrm{C} \mathrm{NMR,} 126 \mathrm{MHz}, \mathrm{CDCl}_{3}$

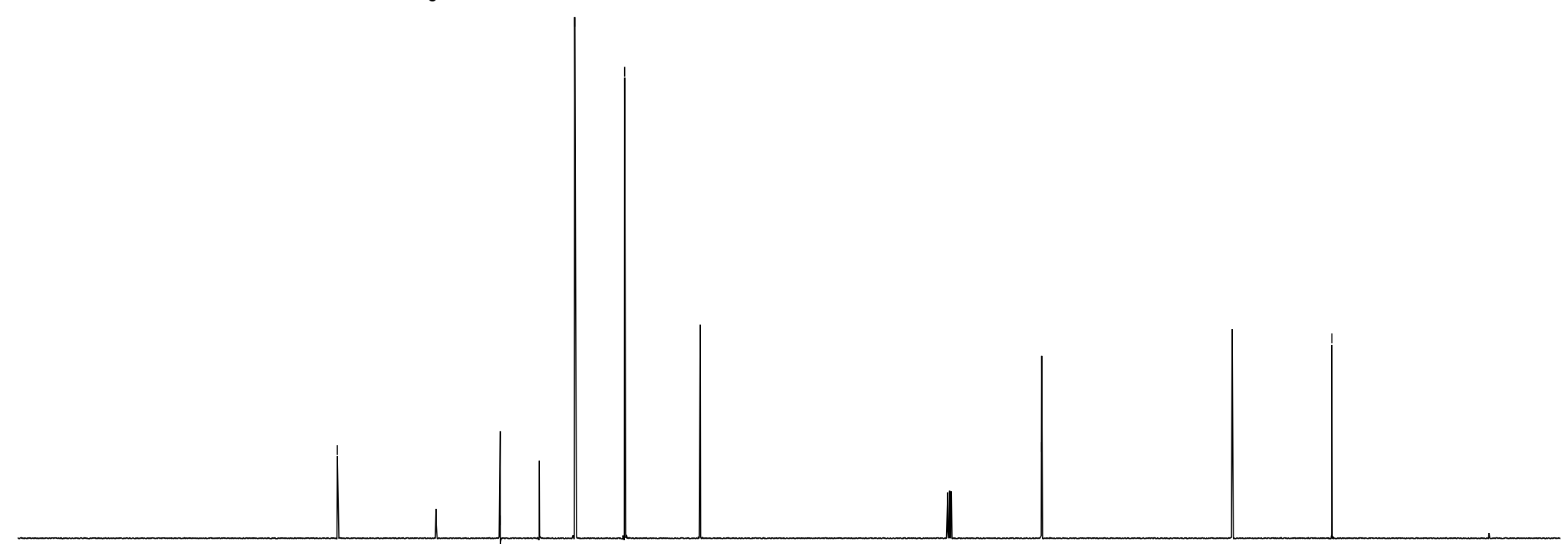

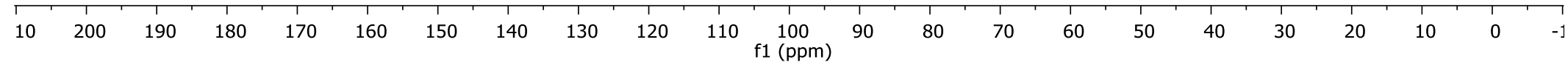



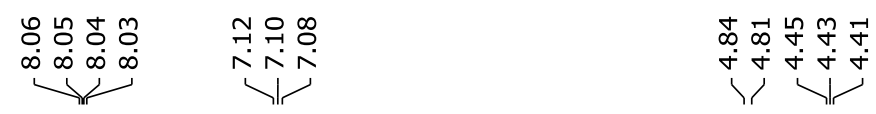

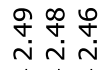

$\stackrel{\substack{-1 \\-i}}{1}$

I

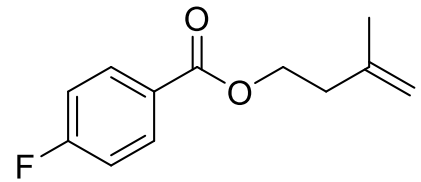

$1 \mathrm{e}$

${ }^{1} \mathrm{H}$ NMR, $400 \mathrm{MHz}, \mathrm{CDCl}_{3}$

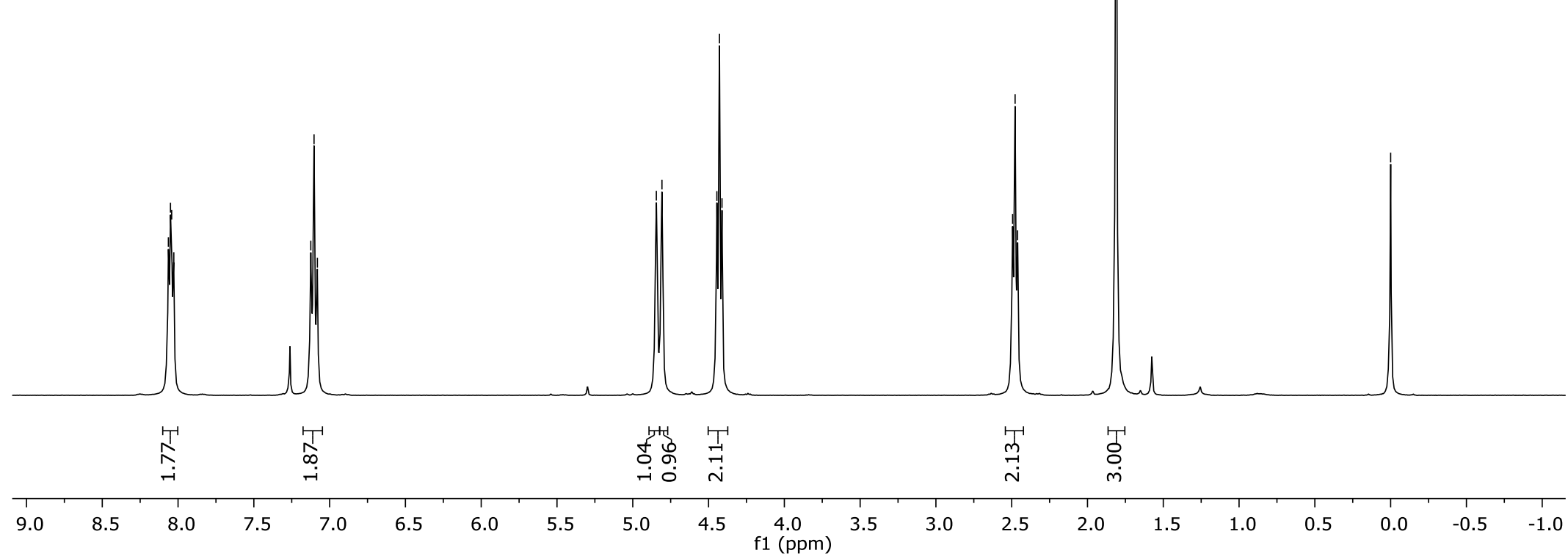




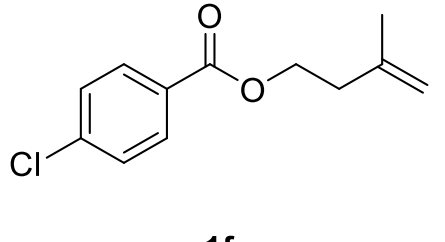

${ }^{1} \mathrm{H} \mathrm{NMR}, 500 \mathrm{MHz}, \mathrm{CDCl}_{3}$

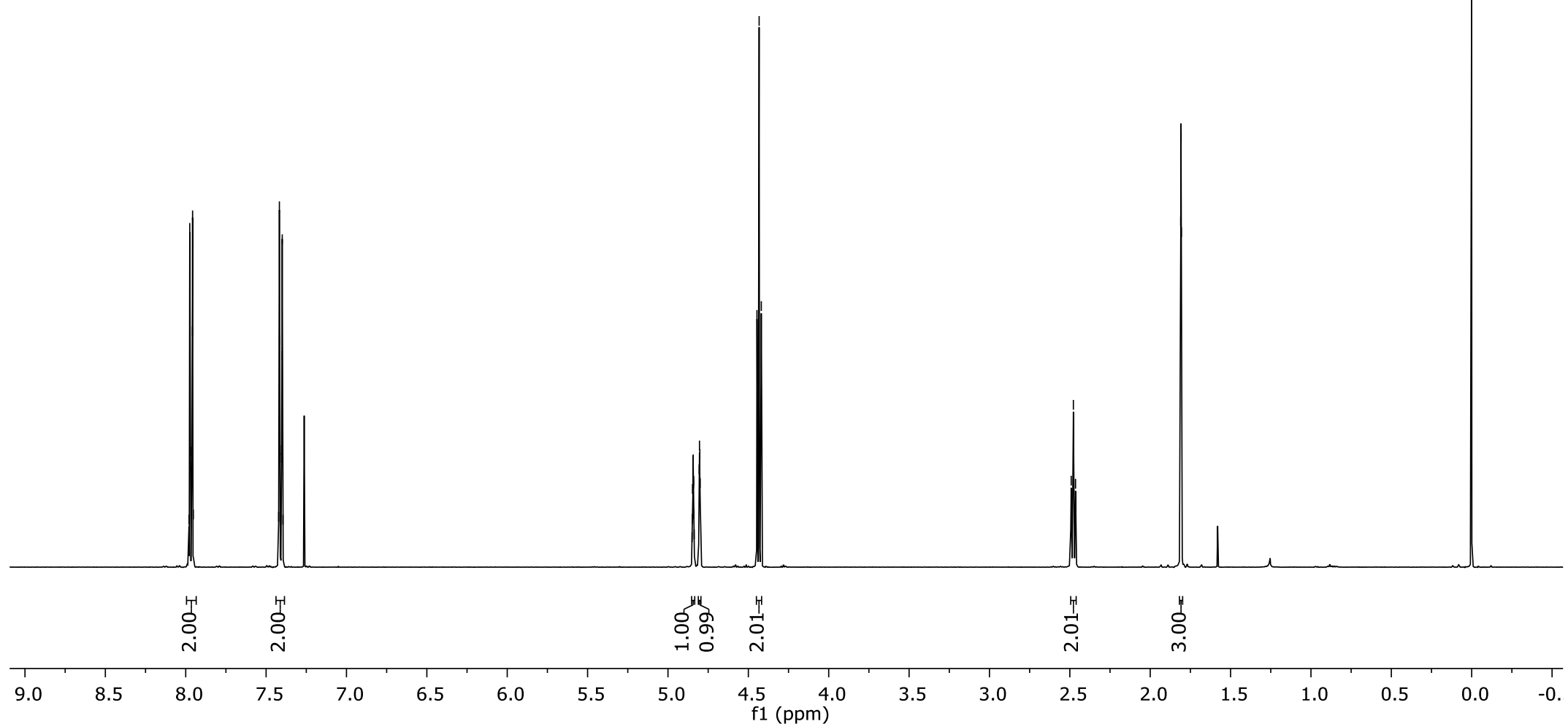




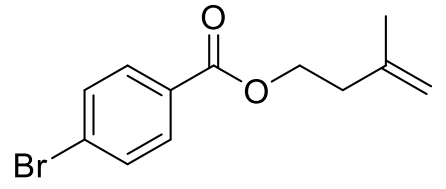

$1 \mathrm{~g}$

${ }^{1} \mathrm{H} \mathrm{NMR}, 500 \mathrm{MHz}, \mathrm{CDCl}_{3}$

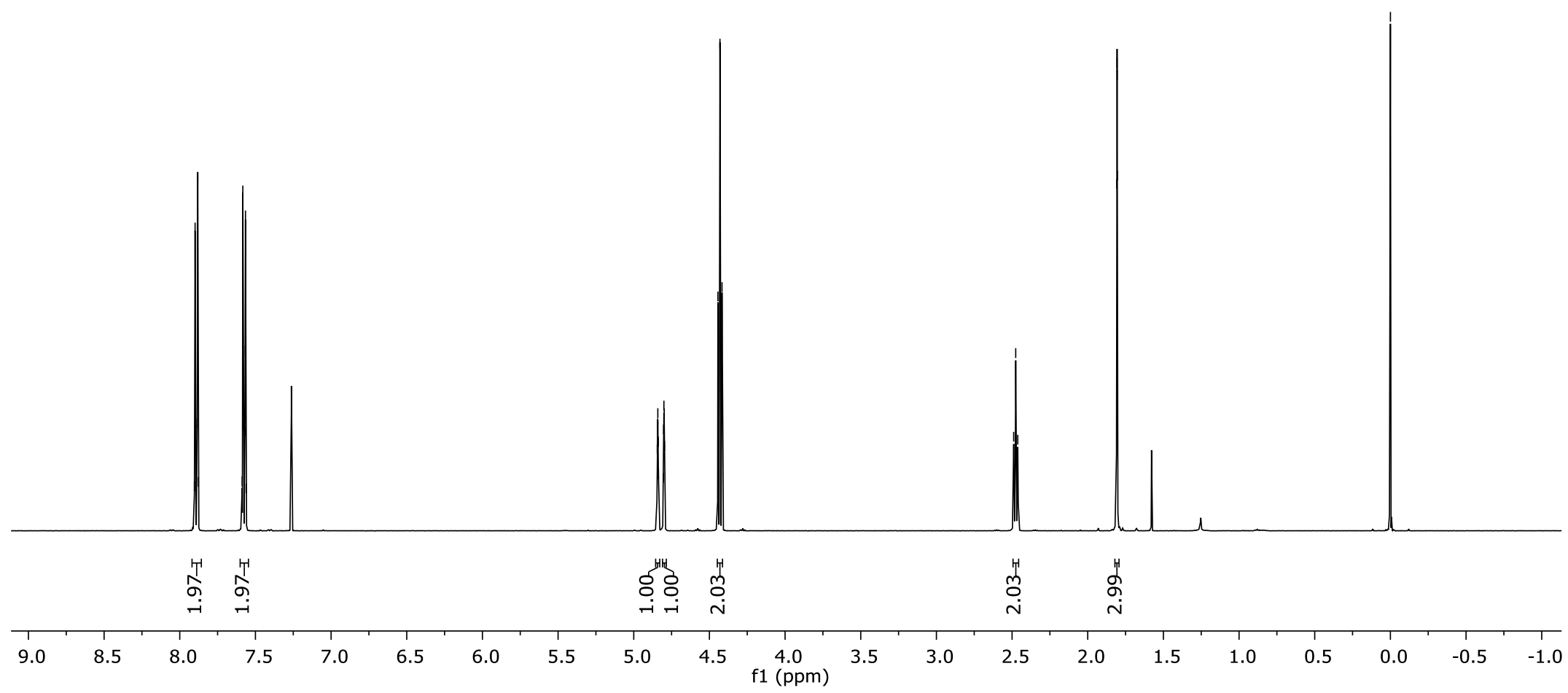


จุ

ن

8

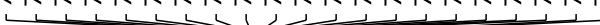

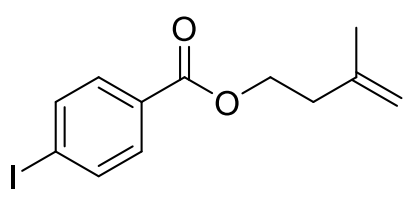

$1 \mathrm{~h}$

${ }^{1} \mathrm{H}$ NMR, $400 \mathrm{MHz}, \mathrm{CDCl}_{3}$

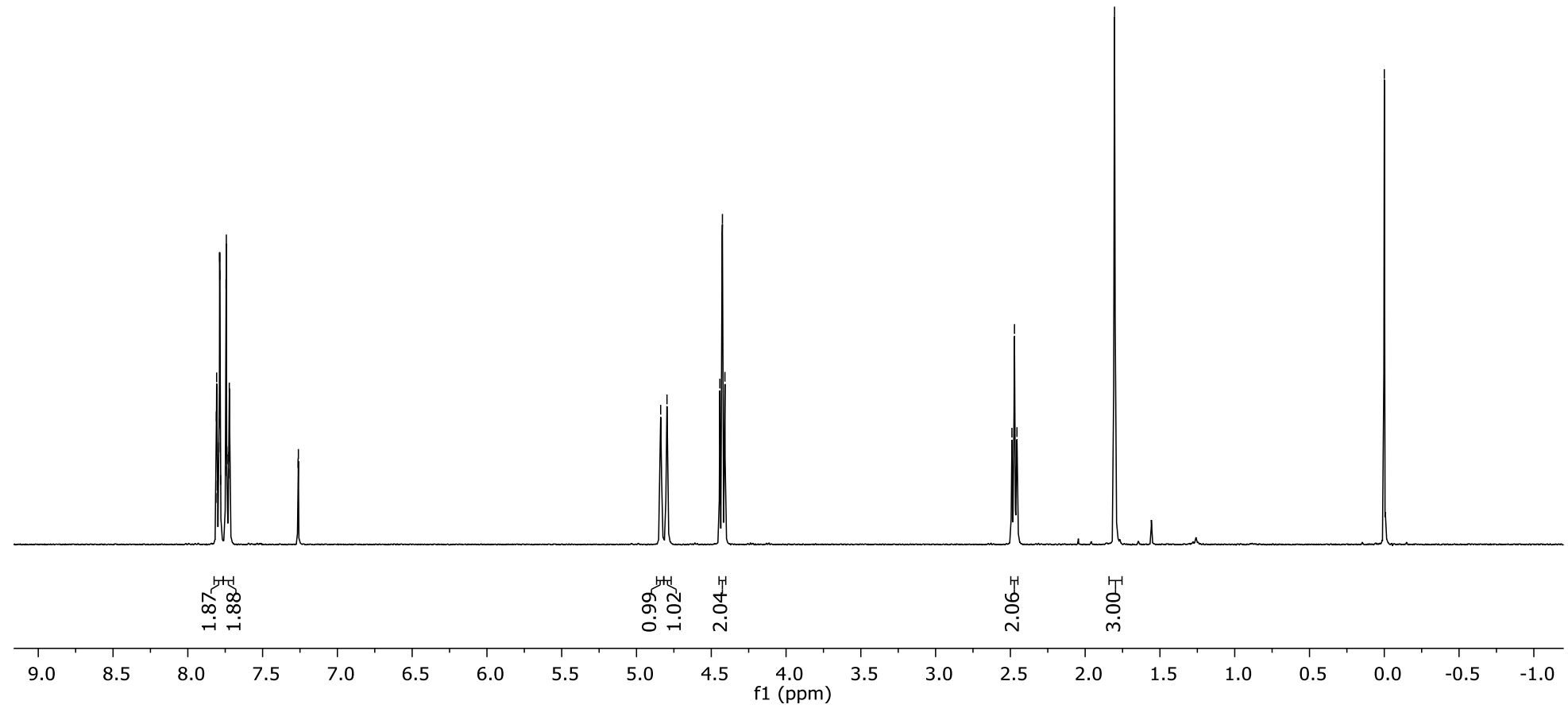


$\begin{array}{cc}\infty & 0 \\ +\infty & 0 \\ \infty & \infty \\ 1 & 1\end{array}$<smiles>C=C(C)CCOC(=O)c1cc(C(F)(F)F)cc(C(F)(F)F)c1</smiles>

$1 \mathrm{i}$

${ }^{1} \mathrm{H} \mathrm{NMR}, 500 \mathrm{MHz}, \mathrm{CDCl}_{3}$

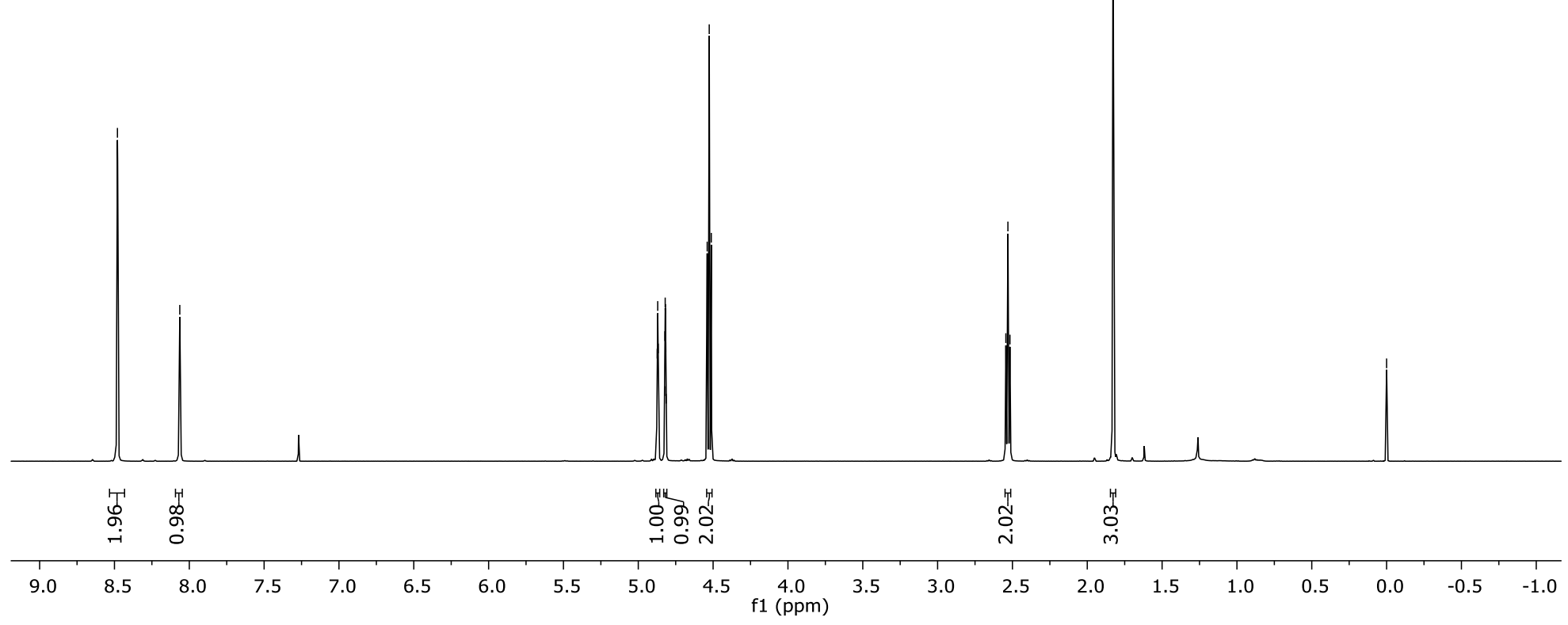




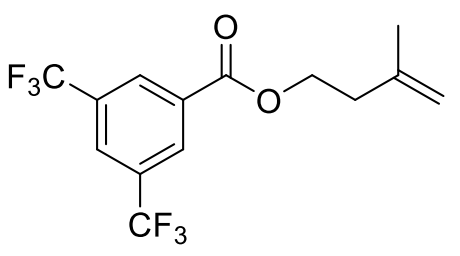

$1 \mathrm{i}$

${ }^{13} \mathrm{C} \mathrm{NMR,} 126 \mathrm{MHz}, \mathrm{CDCl}_{3}$

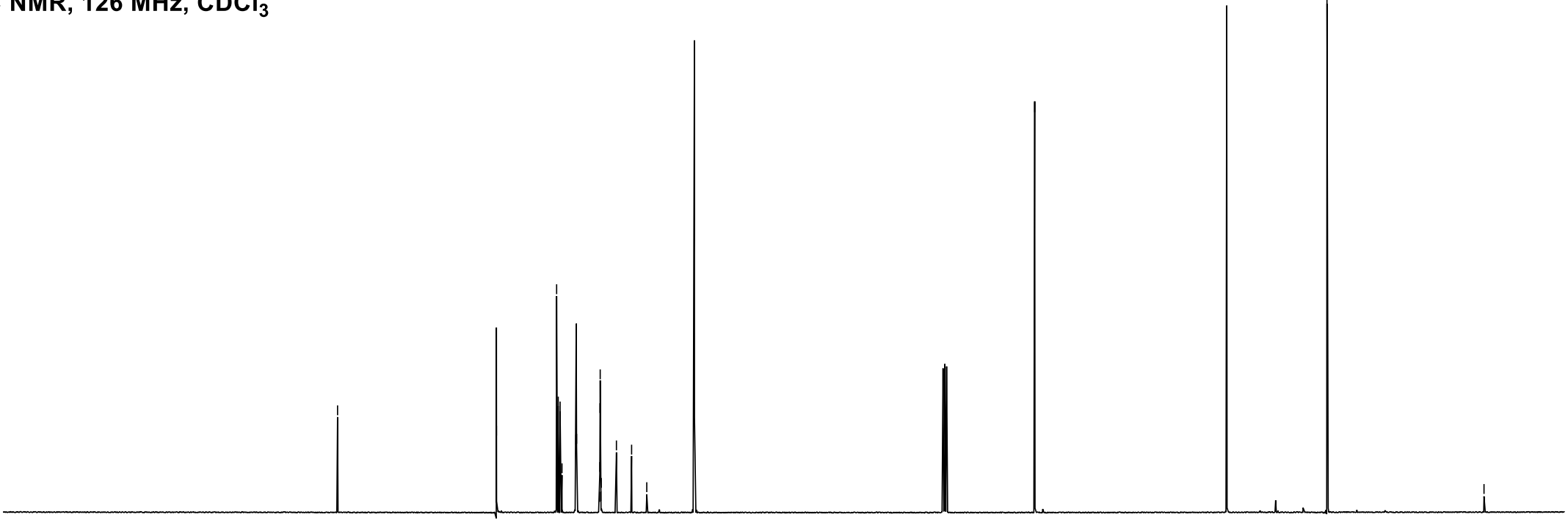




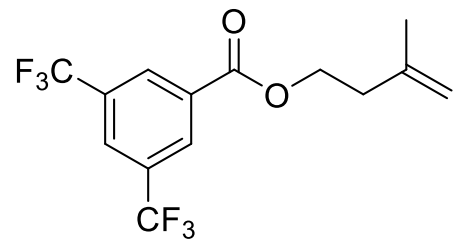

$1 i$

${ }^{19} \mathrm{~F} \mathrm{NMR}, 470 \mathrm{MHz}^{\mathrm{CDCl}}{ }_{3}$

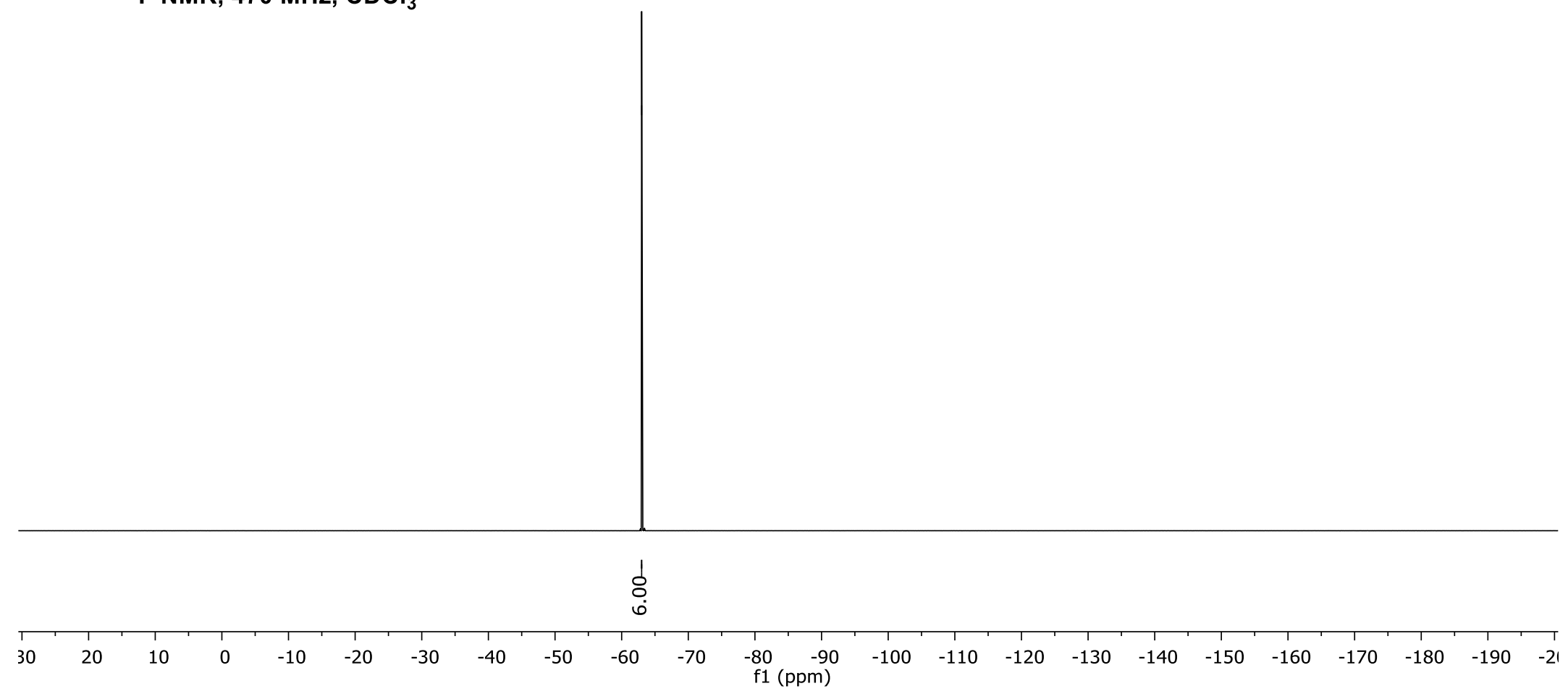




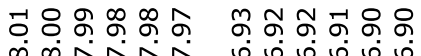

必公公

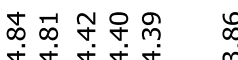

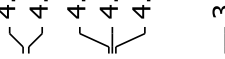<smiles>C=C(C)CCOC(=O)c1ccc(OC)cc1</smiles>

$1 \mathrm{j}$

${ }^{1} \mathrm{H} \mathrm{NMR}, 400 \mathrm{MHz}, \mathrm{CDCl}_{3}$

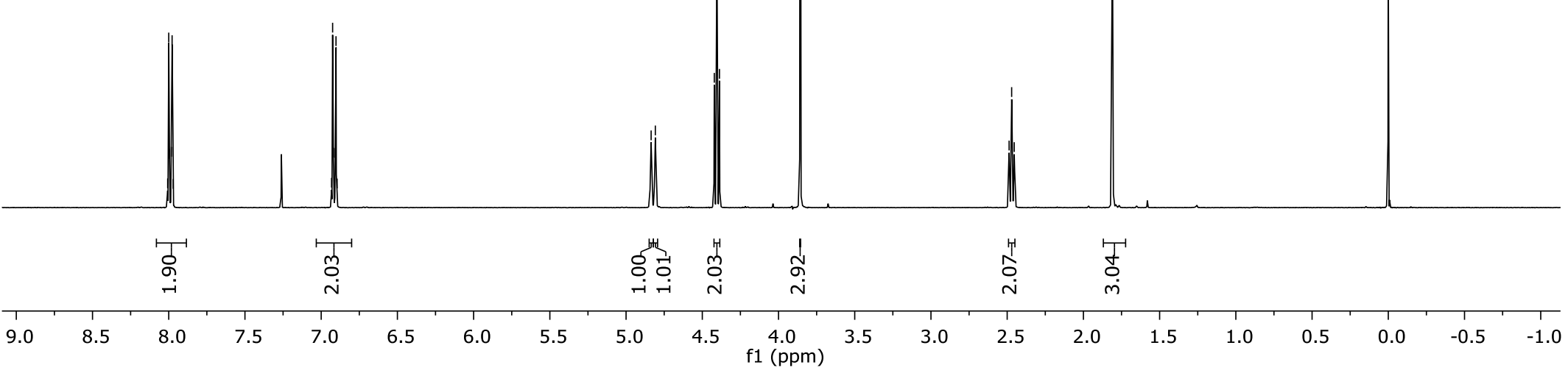




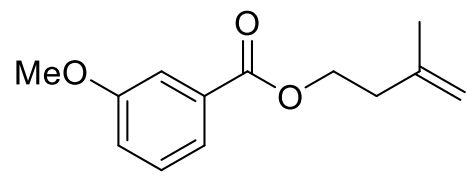

$1 \mathrm{k}$

${ }^{1} \mathrm{H}$ NMR, $400 \mathrm{MHz}, \mathrm{CDCl}_{3}$

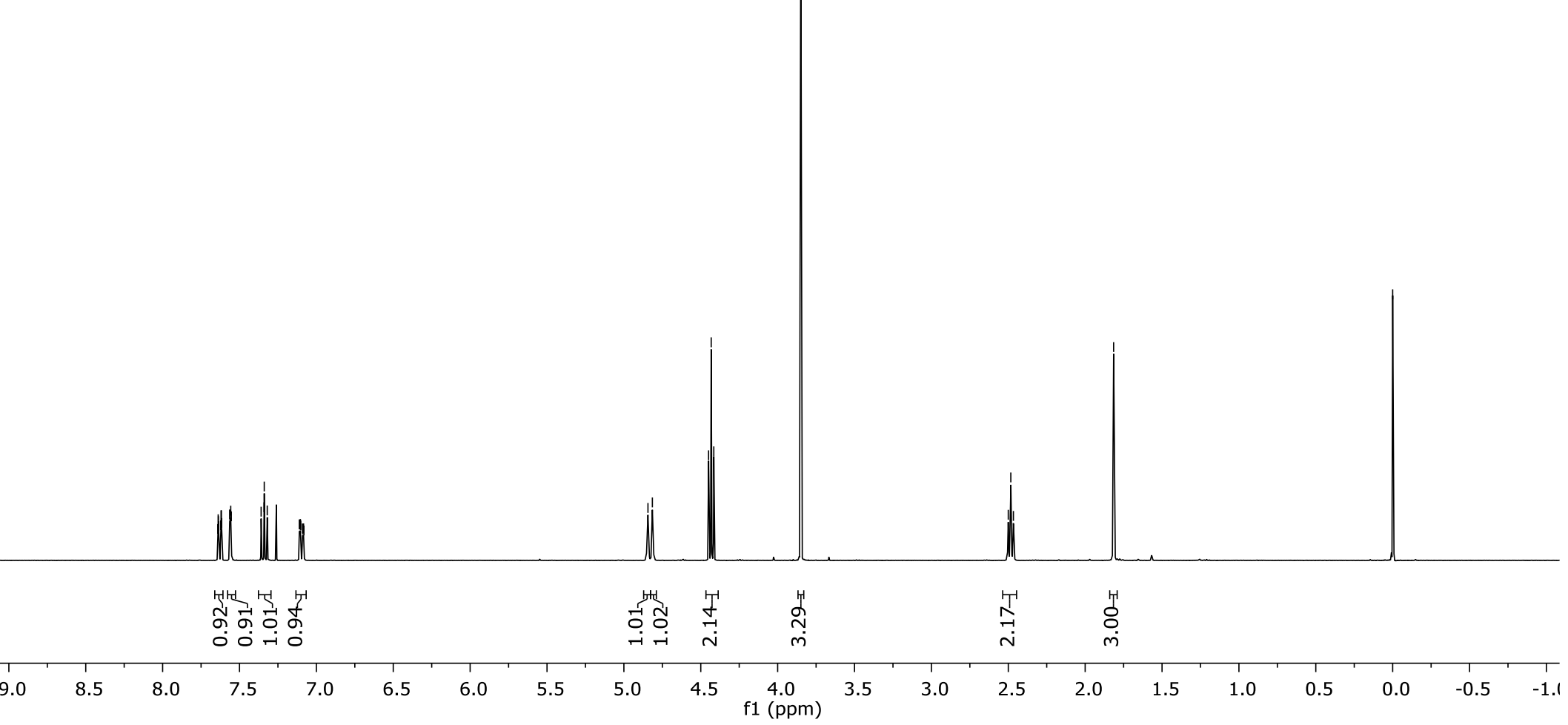




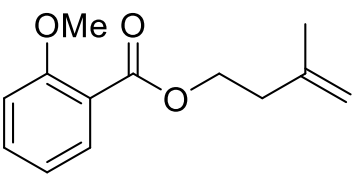

11

${ }^{1} \mathrm{H}$ NMR, $400 \mathrm{MHz}, \mathrm{CDCl}_{3}$

\section{NMR, $400 \mathrm{MHz}, \mathrm{CDCl}_{3}$}

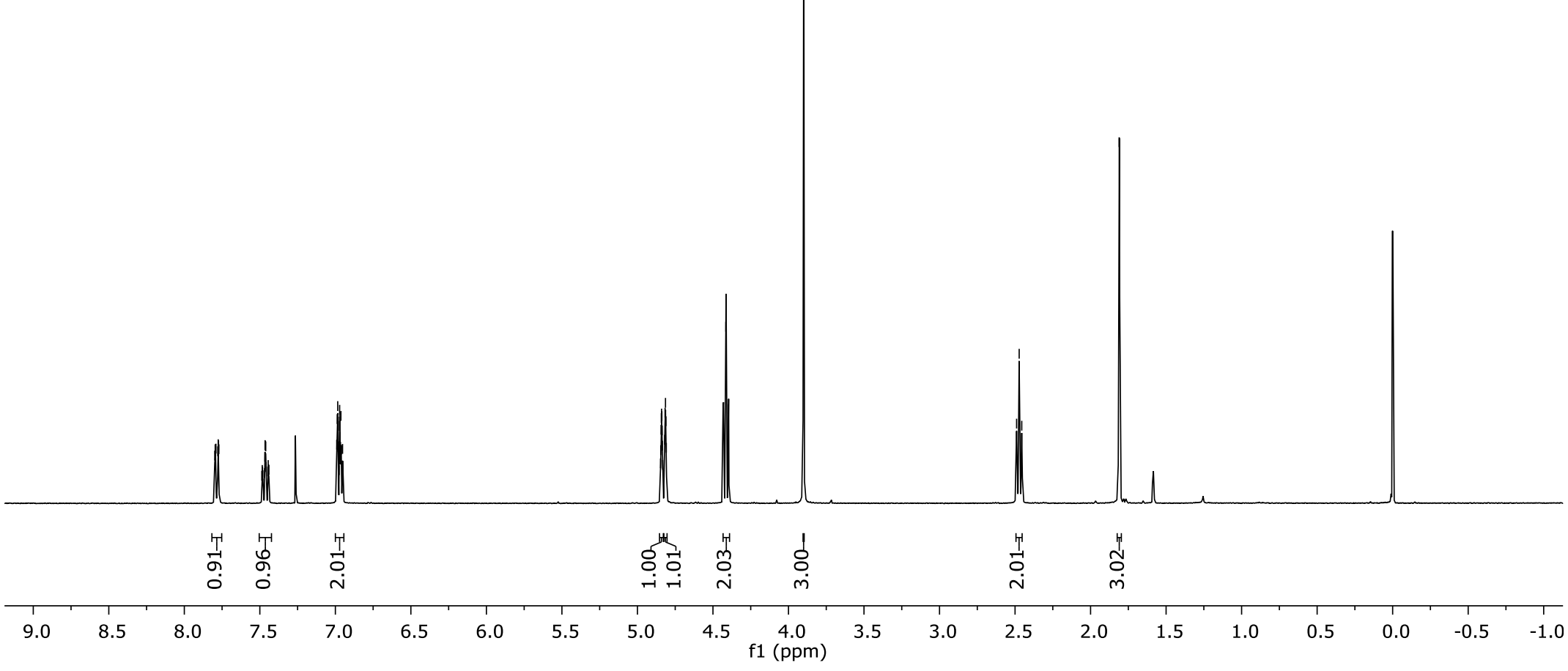


䠉

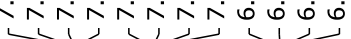

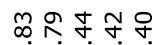

$\dot{i} \dot{d}$

$\operatorname{did}$

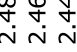

$\stackrel{\infty}{\stackrel{\infty}{+}}$<smiles>C=C(C)CCOC(=O)c1ccco1</smiles>

$1 \mathrm{~m}$

${ }^{1} \mathrm{H} \mathrm{NMR}, 400 \mathrm{MHz}, \mathrm{CDCl}_{3}$

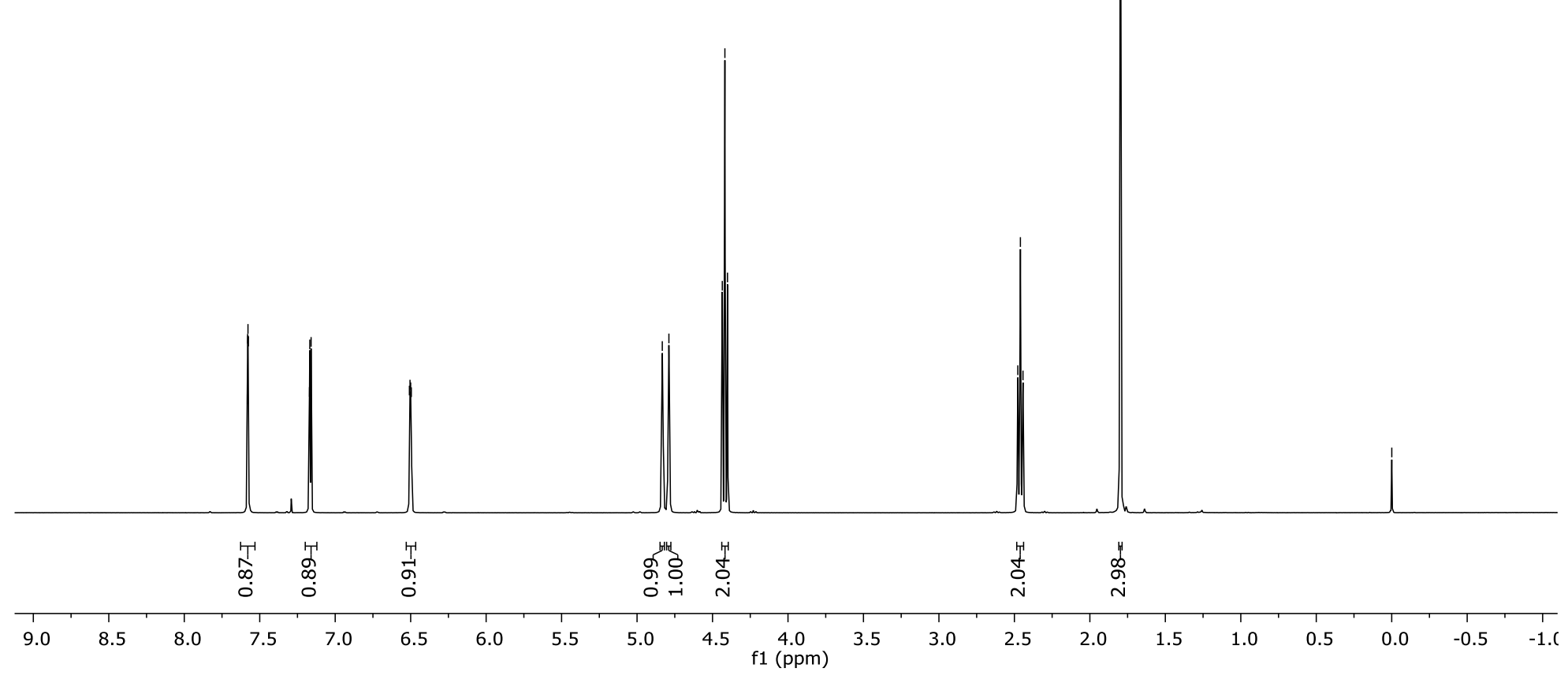


SI-38

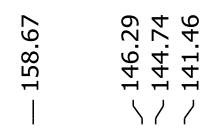

1

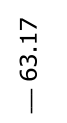

$\stackrel{\substack{n \\ \stackrel{0}{n}}}{i}$

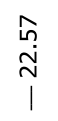

$\stackrel{8}{i}$

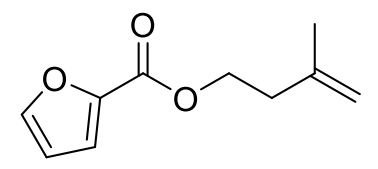

$1 \mathrm{~m}$

${ }^{13} \mathrm{C} \mathrm{NMR}, 101 \mathrm{MHz}, \mathrm{CDCl}_{3}$

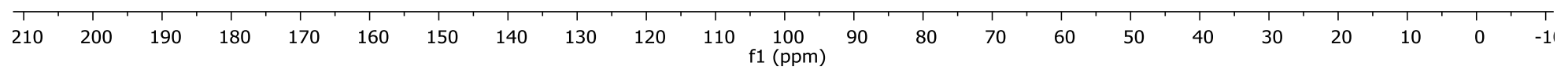




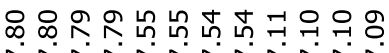

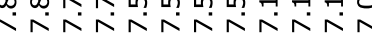

亦

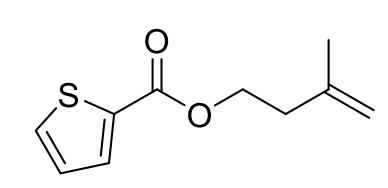

$1 \mathrm{n}$

${ }^{1} \mathrm{H}$ NMR, $500 \mathrm{MHz}, \mathrm{CDCl}_{3}$

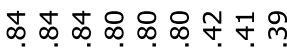

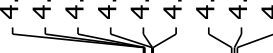

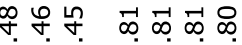

年

$\sqrt{1} \sqrt{2}$

$\stackrel{0}{0}$

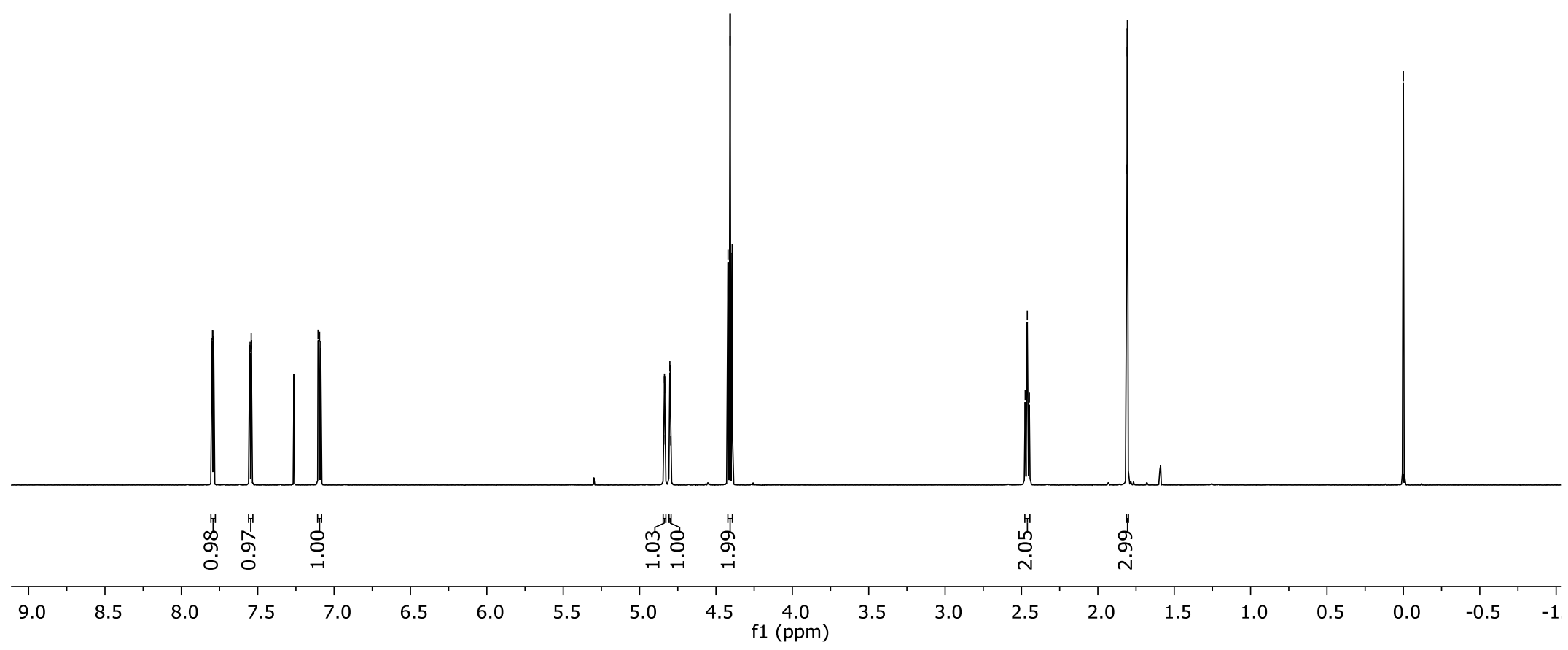




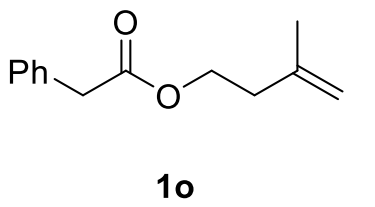

${ }^{1} \mathrm{H}$ NMR, $400 \mathrm{MHz}, \mathrm{CDCl}_{3}$

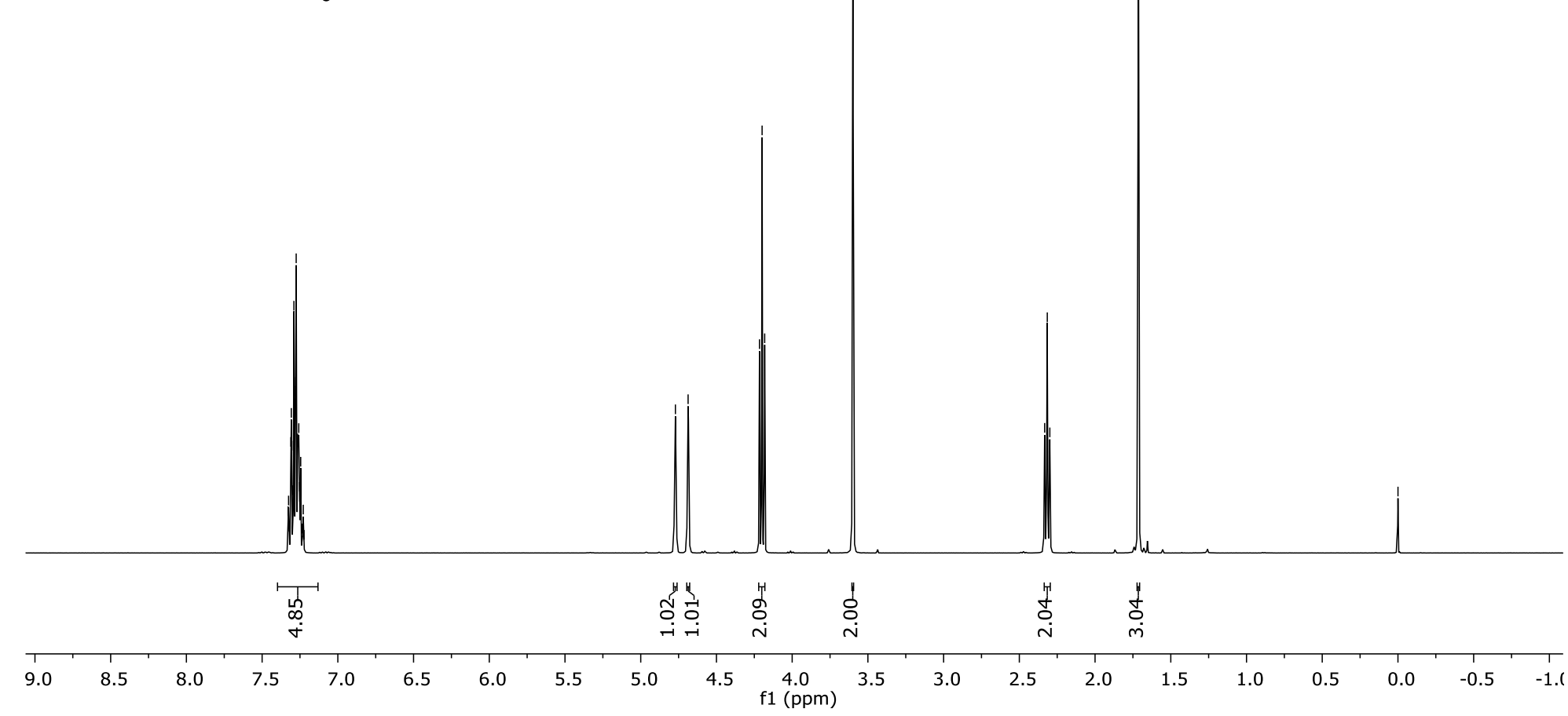




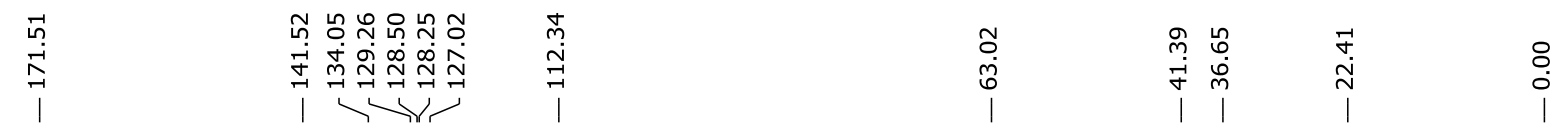

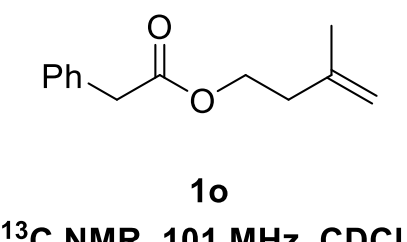

${ }^{\mathrm{C}} \mathrm{NMR}, 101 \mathrm{MHz}, \mathrm{CDCl}_{3}$
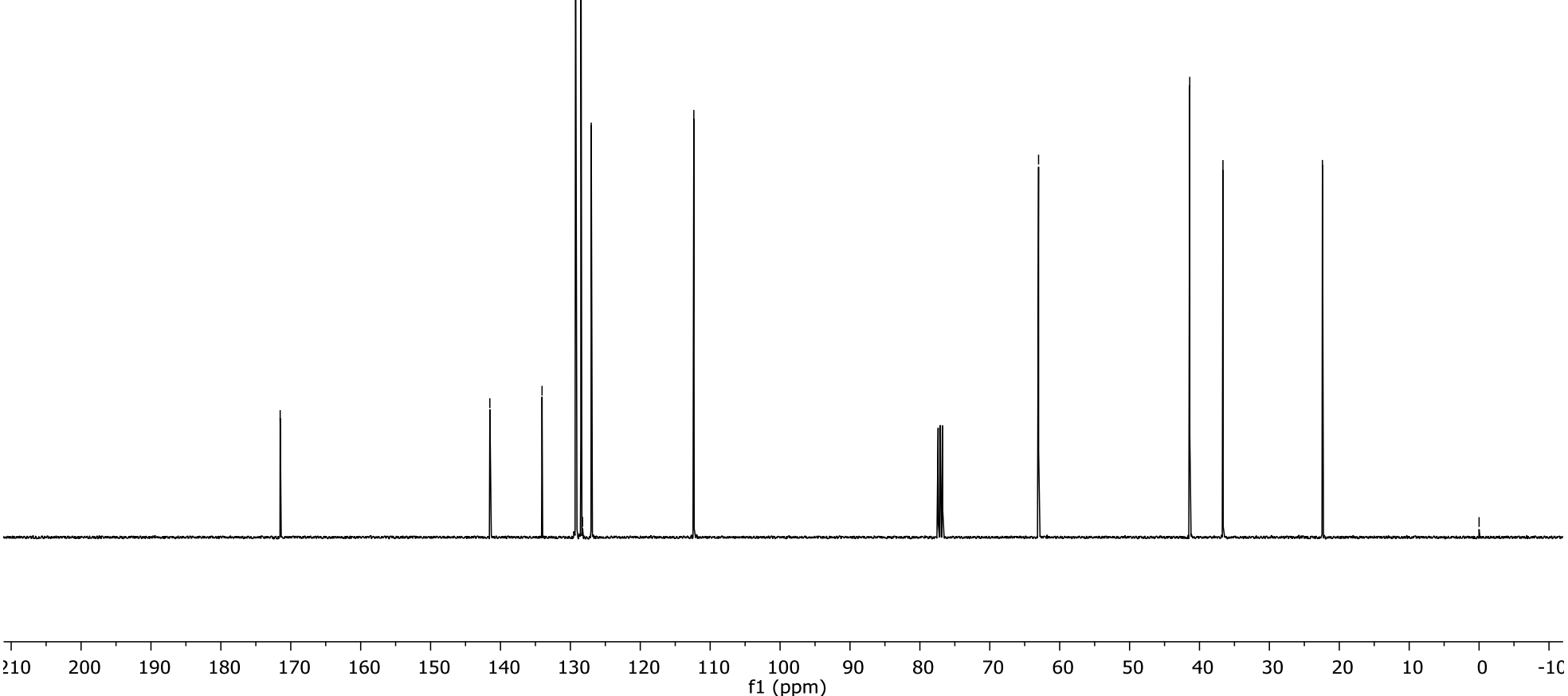


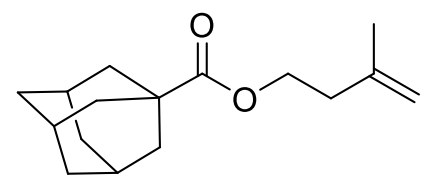

$1 \mathrm{p}$

${ }^{1} \mathrm{H}$ NMR, $400 \mathrm{MHz}, \mathrm{CDCl}_{3}$

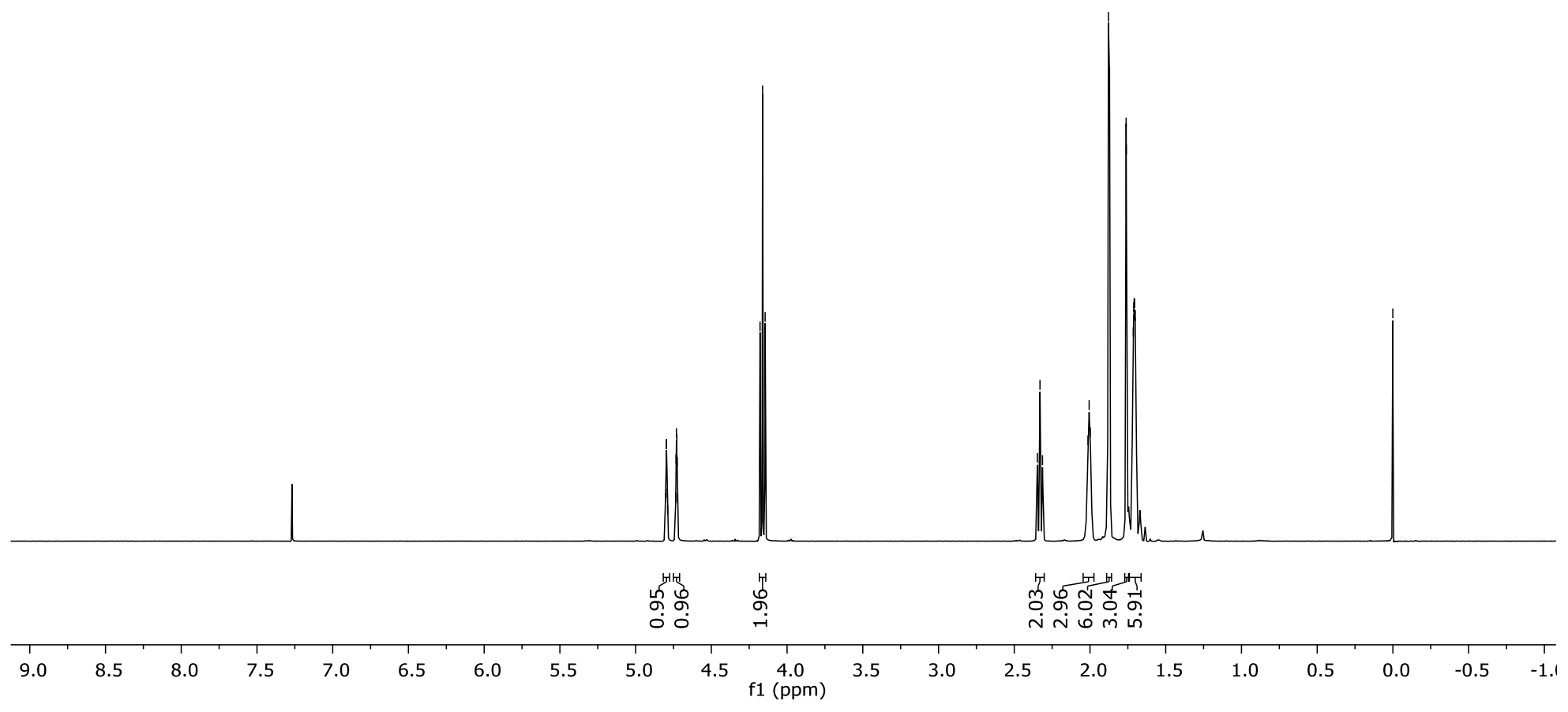




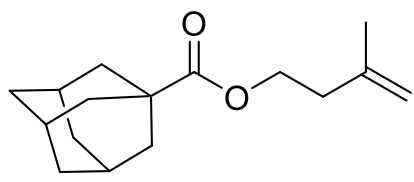

$1 p$

${ }^{13} \mathrm{C} \mathrm{NMR,}, 101 \mathrm{MHz}^{\mathrm{CDCl}} \mathrm{C}_{3}$
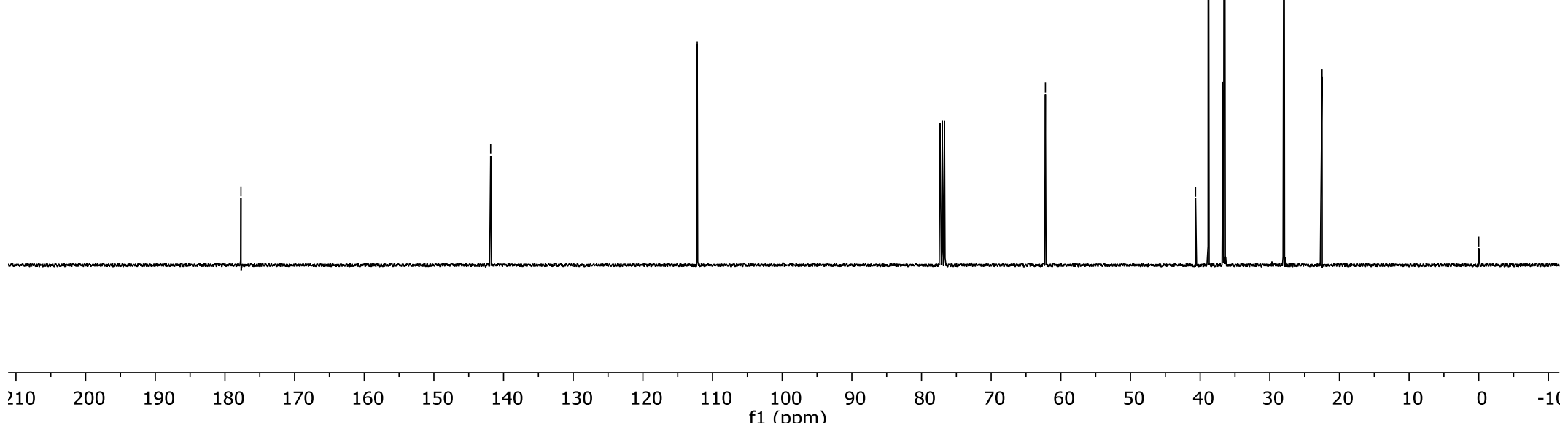


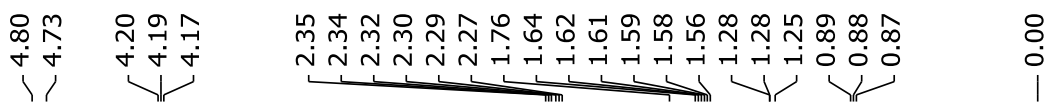

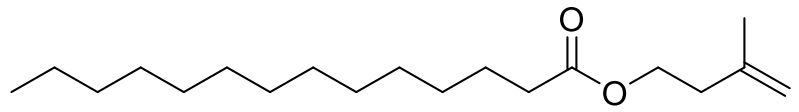

$1 q$

${ }^{1} \mathrm{H}$ NMR, $500 \mathrm{MHz}, \mathrm{CDCl}_{3}$

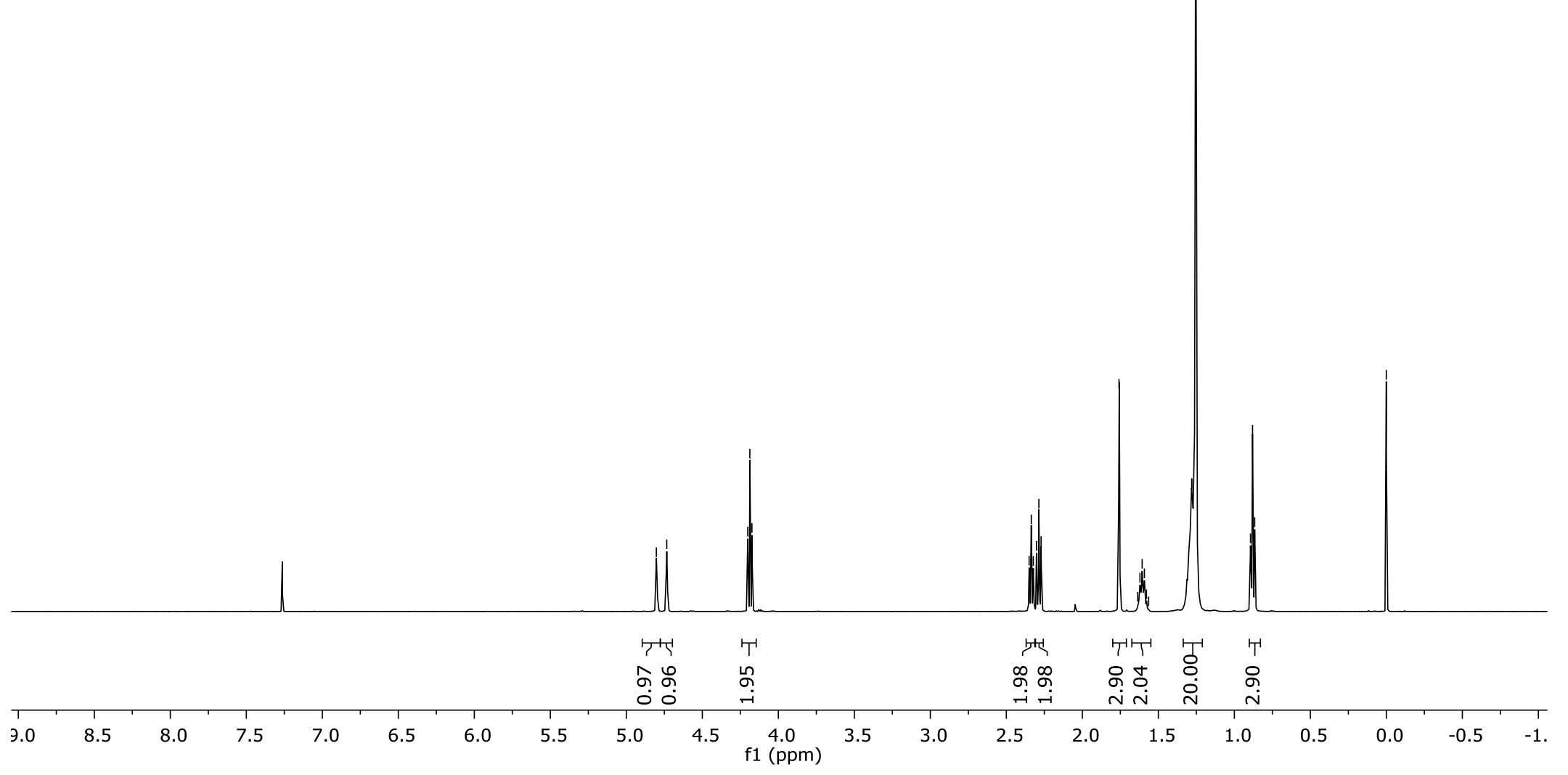




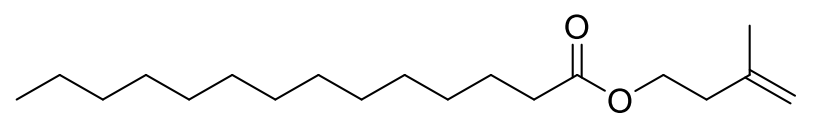

19

${ }^{13} \mathrm{C} \mathrm{NMR}, 126 \mathrm{MHz} \mathrm{CDCl}_{3}$
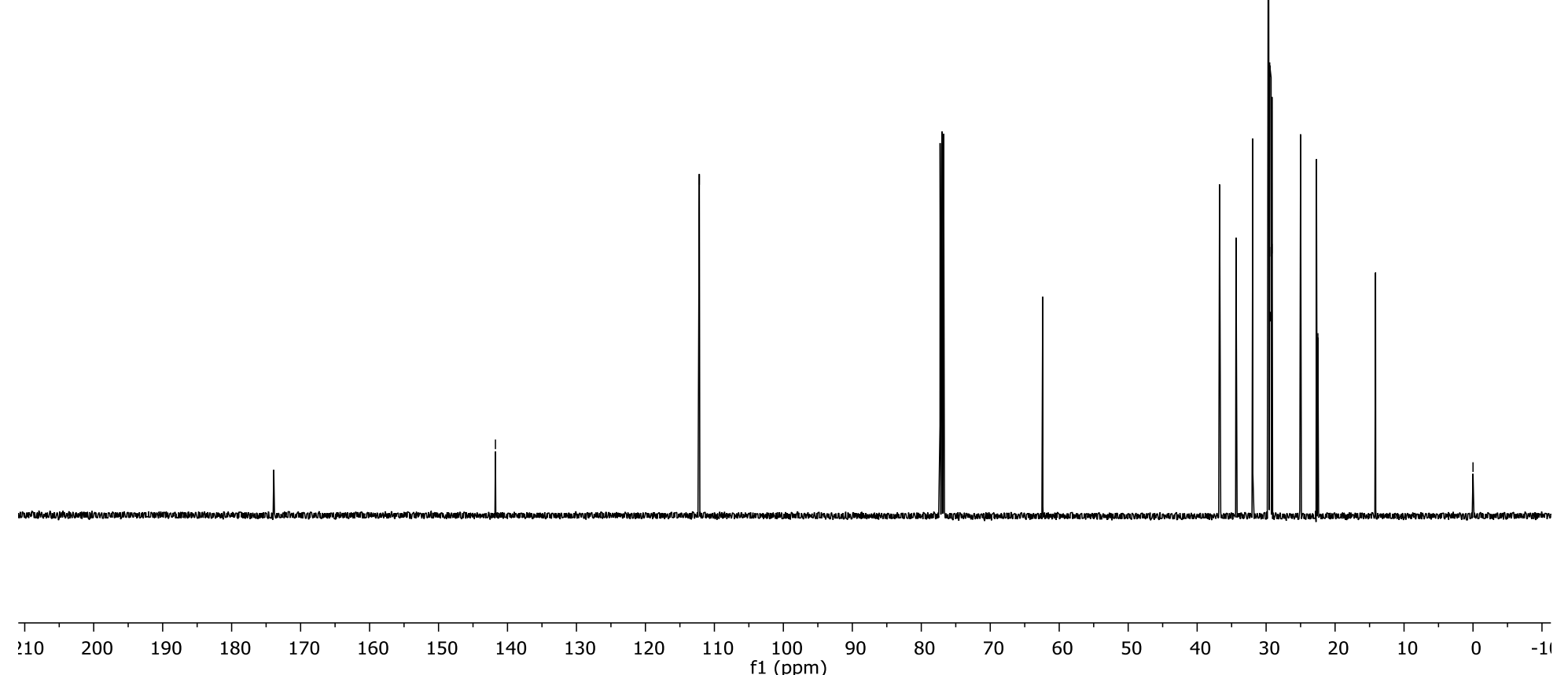


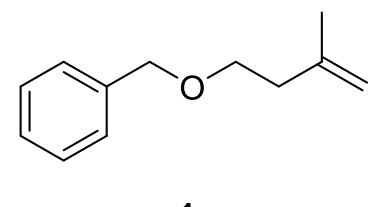

${ }^{1} \mathrm{H}$ NMR, $500 \mathrm{MHz}, \mathrm{CDCl}_{3}$

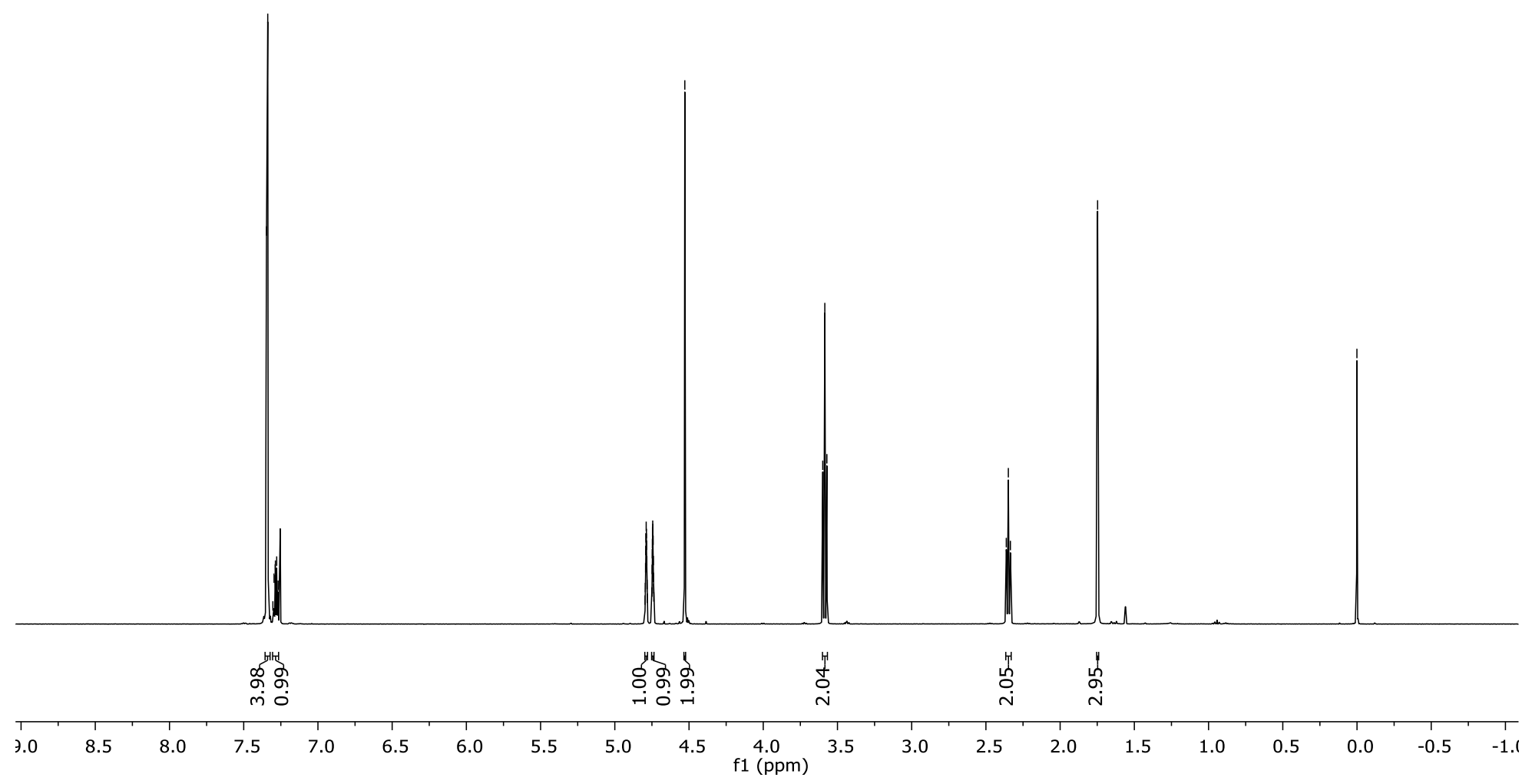




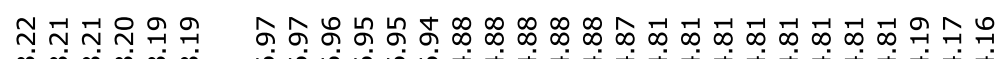

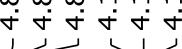

英焕

NN Hन-1-T

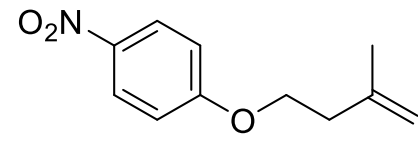

$1 \mathrm{~s}$

${ }^{1} \mathrm{H}$ NMR, $500 \mathrm{MHz}, \mathrm{CDCl}_{3}$

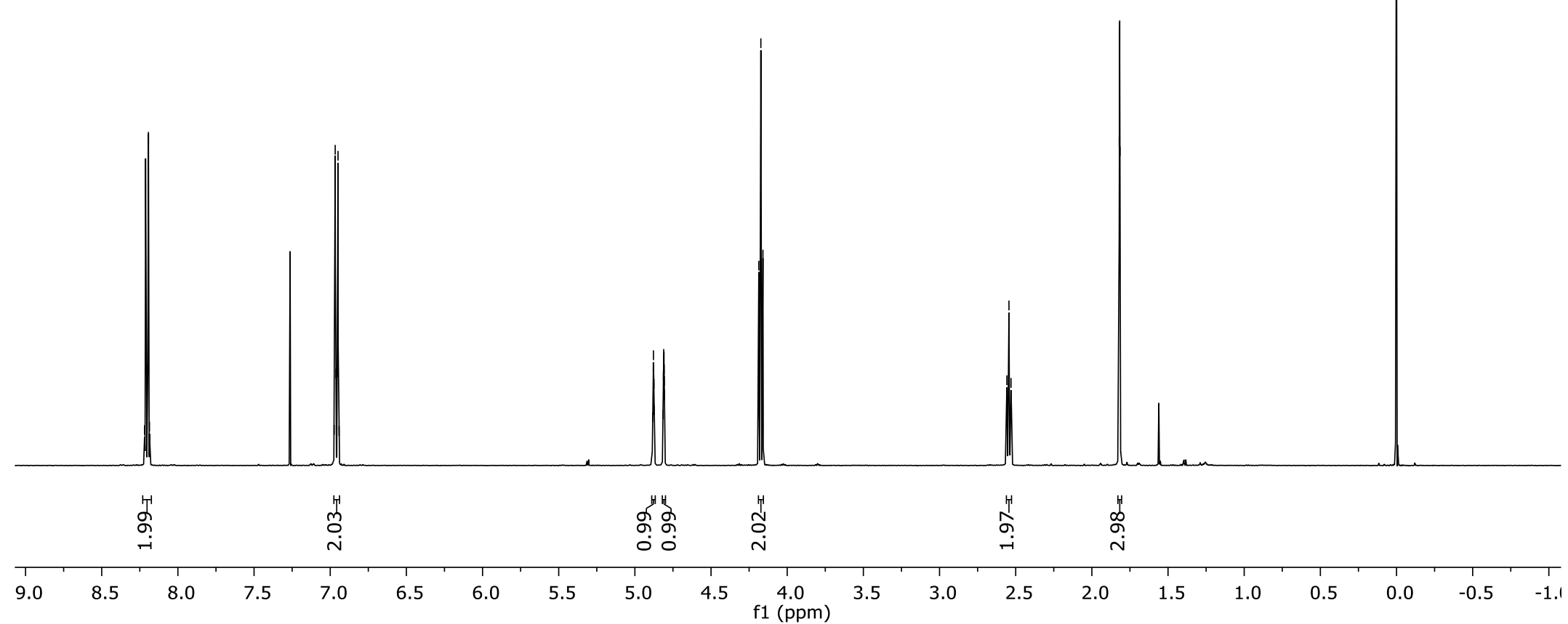




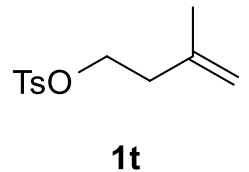

${ }^{1} \mathrm{H} \mathrm{NMR}, 400 \mathrm{MHz}, \mathrm{CDCl}_{3}$

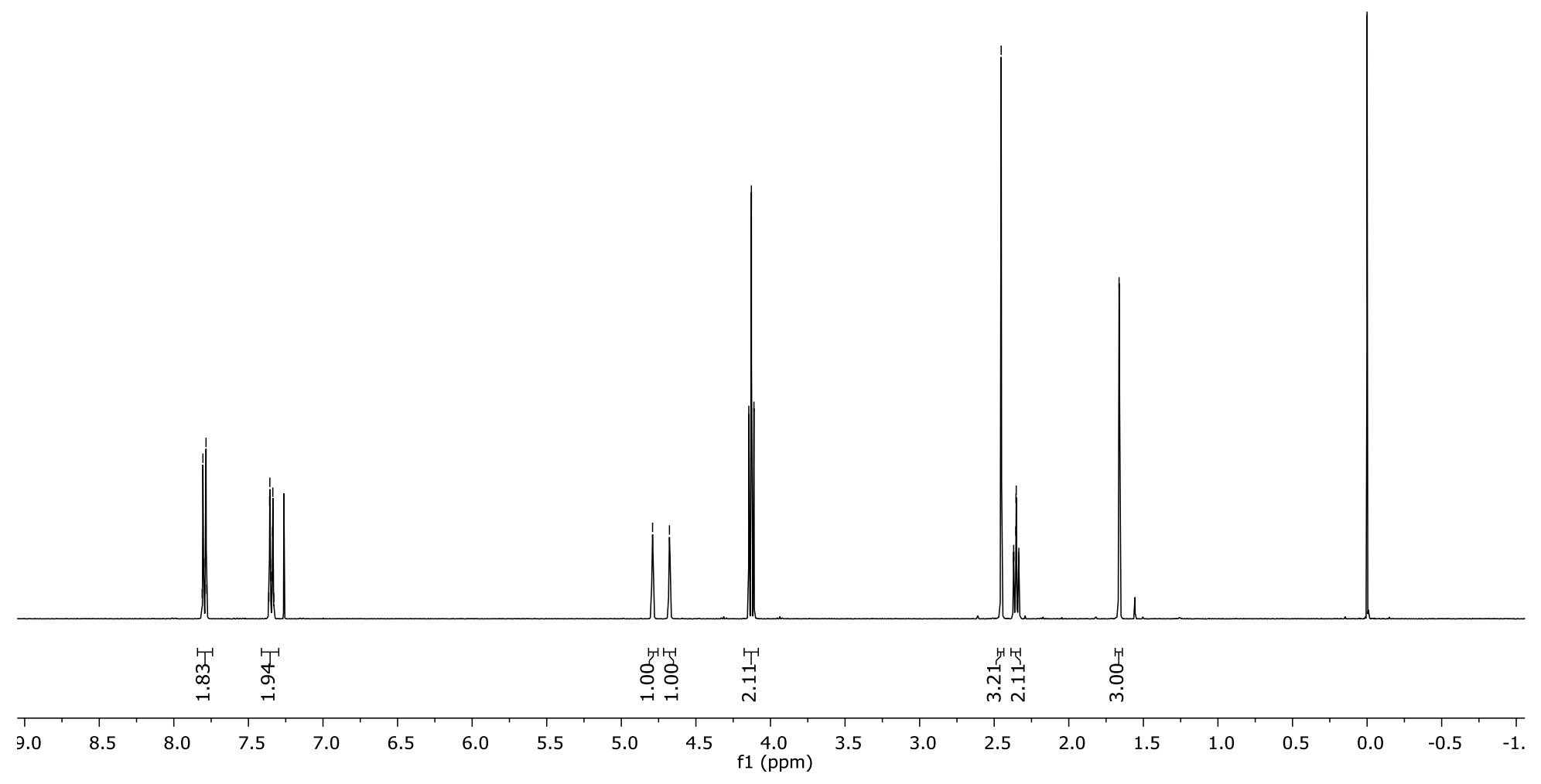




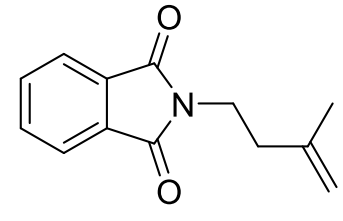

$1 \mathrm{u}$

${ }^{1} \mathrm{H}$ NMR, $500 \mathrm{MHz}, \mathrm{CDCl}_{3}$

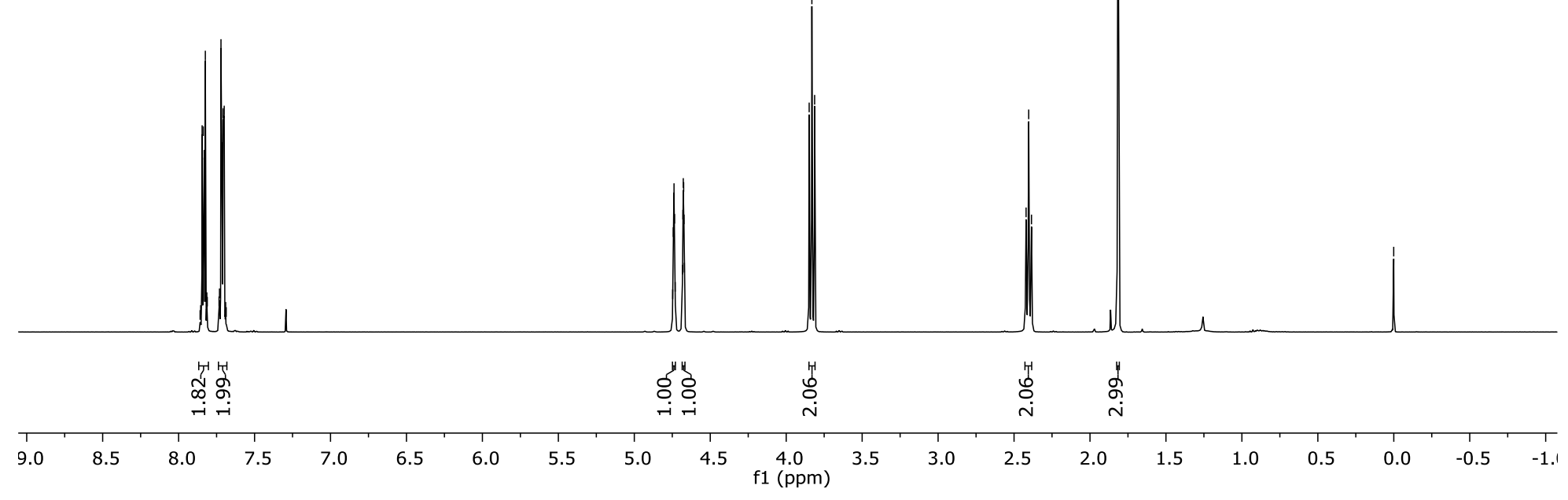




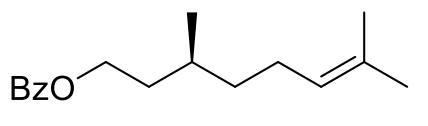

$1 \mathrm{v}$

${ }^{1} \mathrm{H}$ NMR, $500 \mathrm{MHz}, \mathrm{CDCl}_{3}$

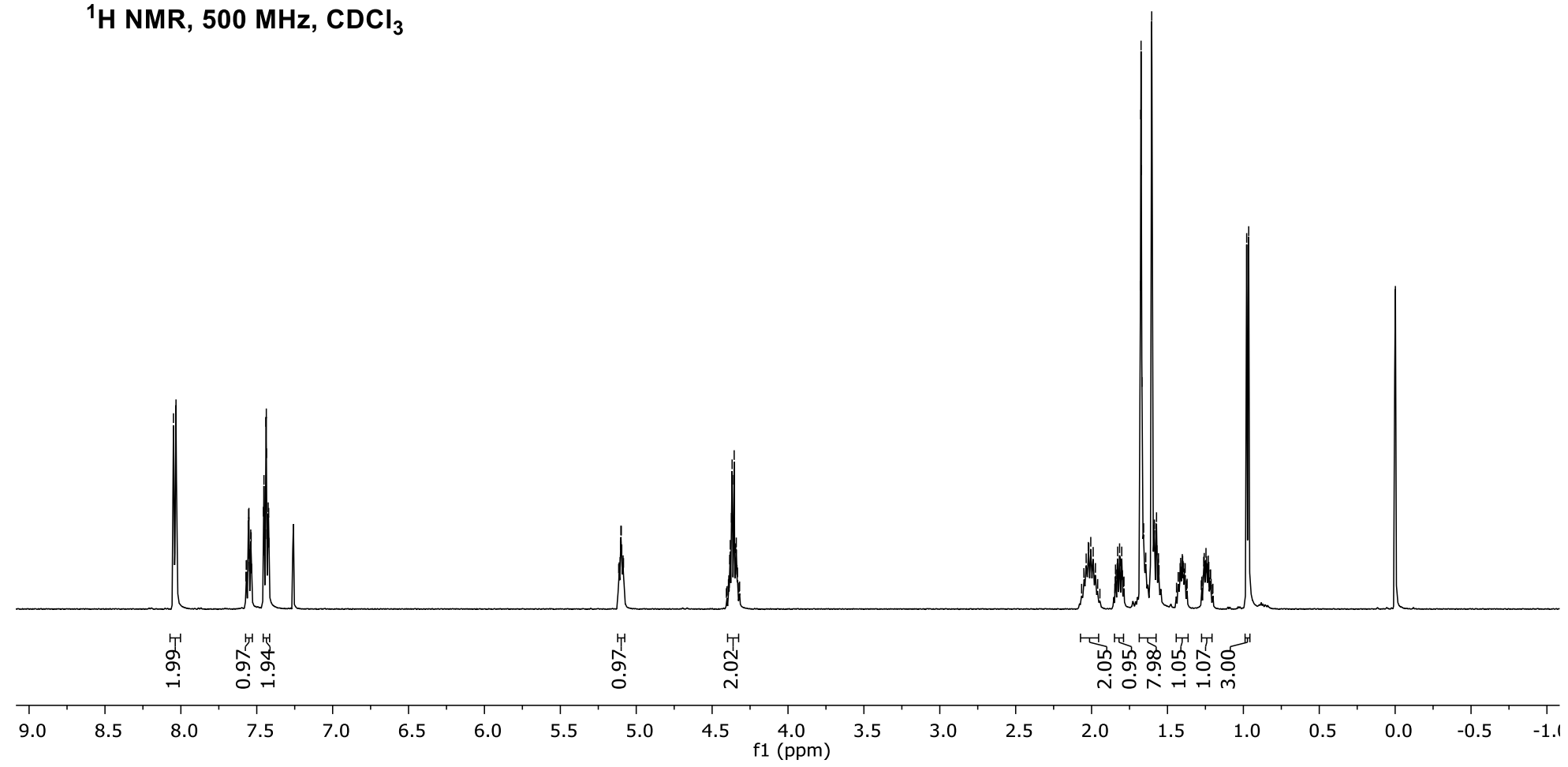




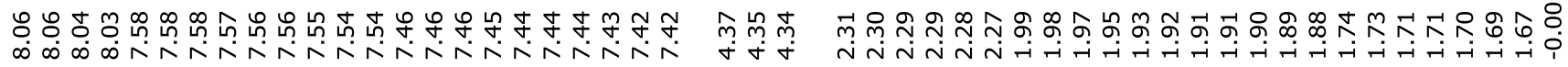

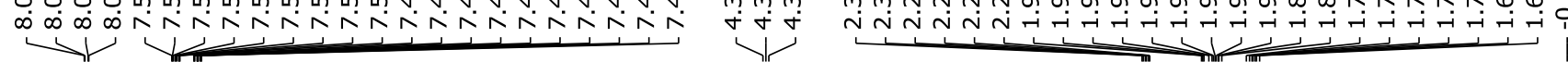

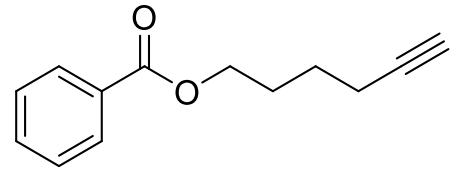

S1

${ }^{1} \mathrm{H} \mathrm{NMR}, 400 \mathrm{MHz}, \mathrm{CDCl}_{3}$

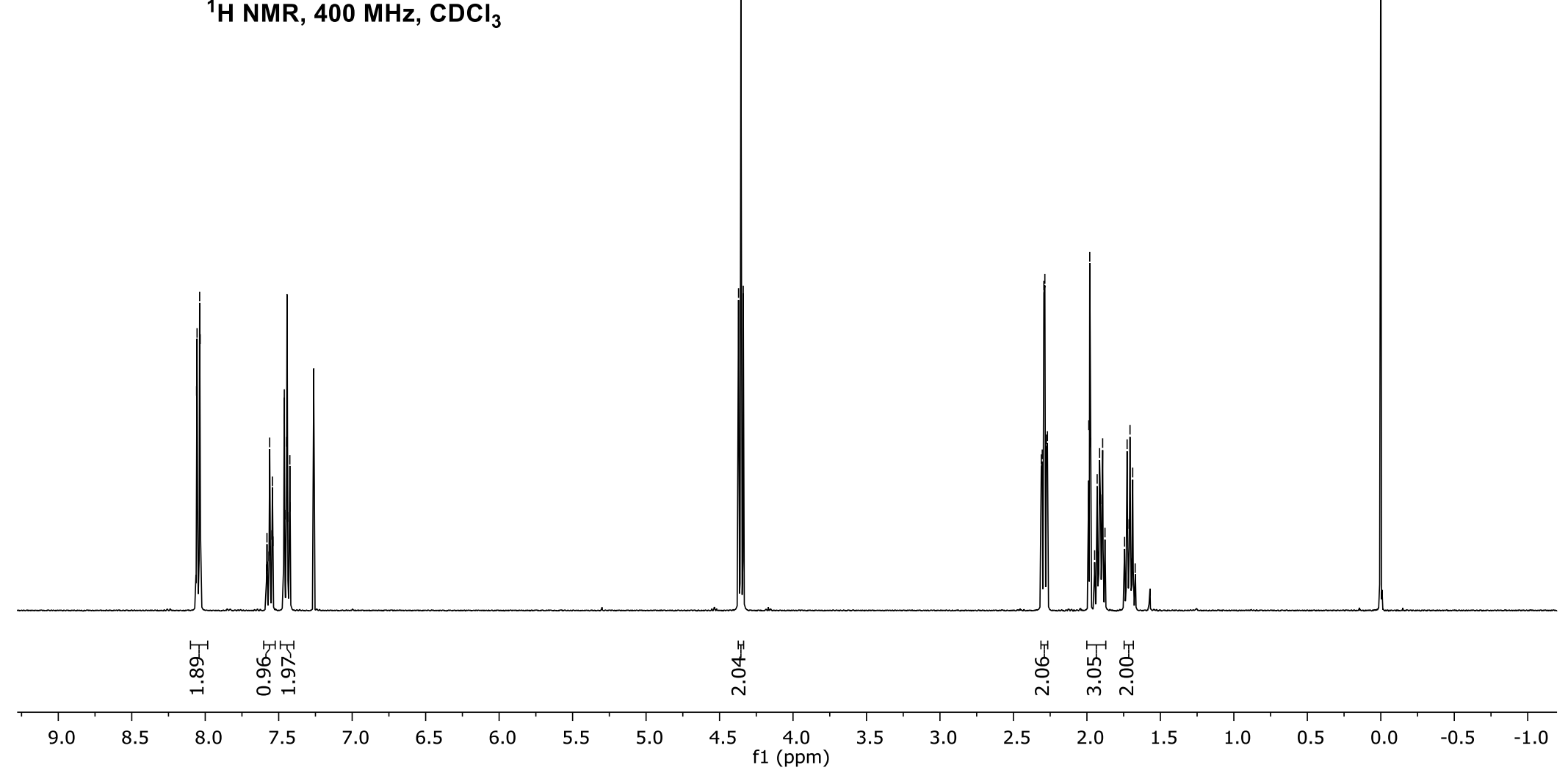




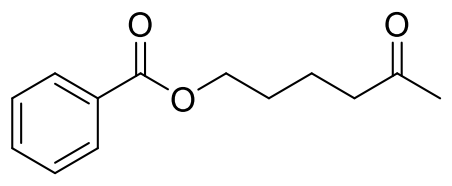

S2

${ }^{1} \mathrm{H}$ NMR, $400 \mathrm{MHz}, \mathrm{CDCl}_{3}$

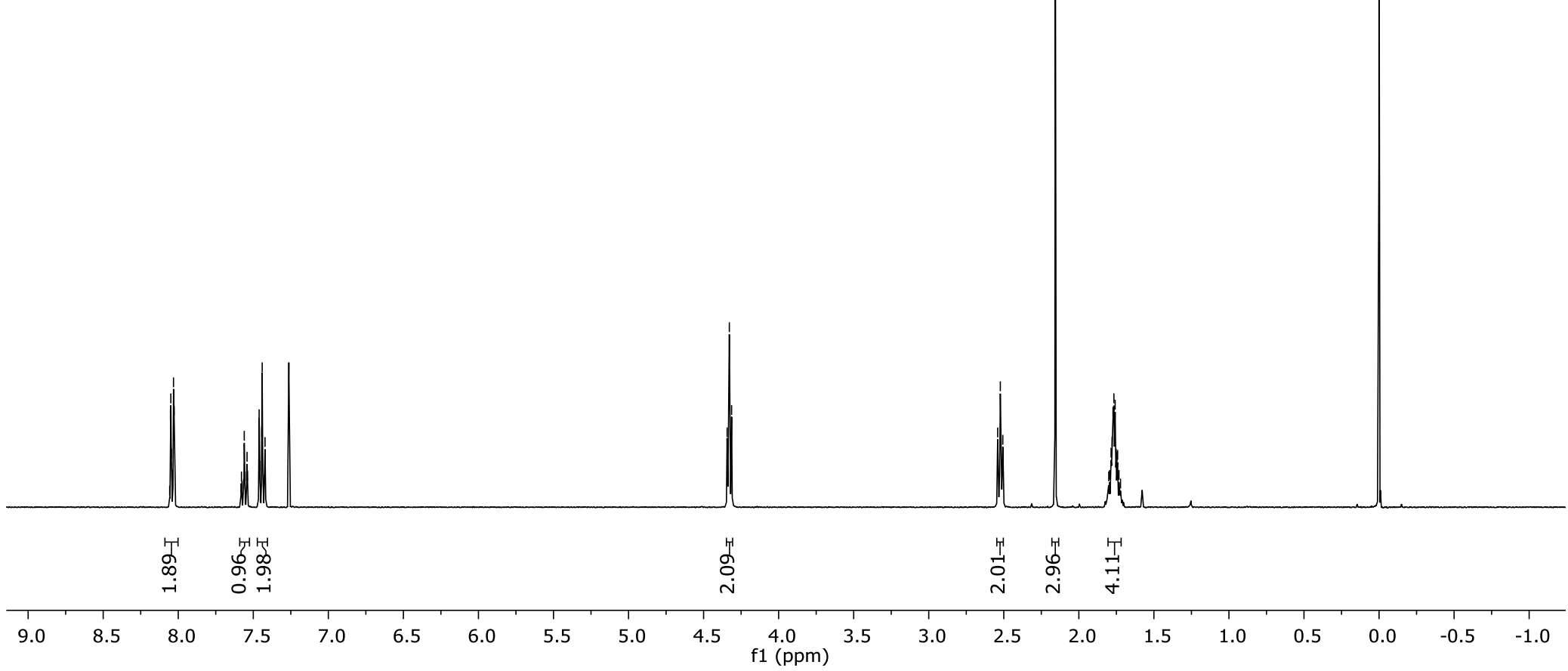


SI-53

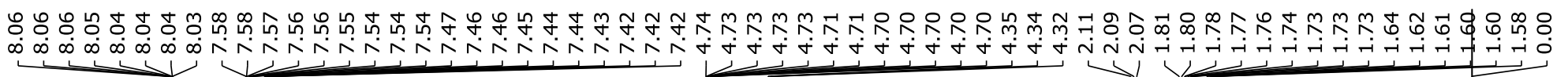<smiles>C=C(C)CCCCOC(=O)c1ccccc1</smiles>
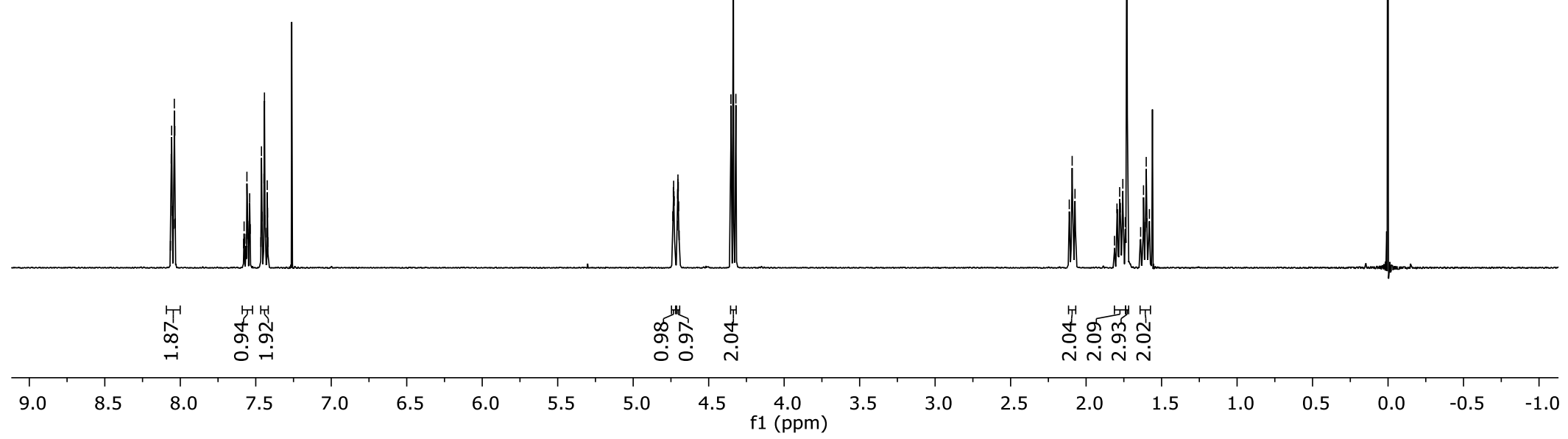


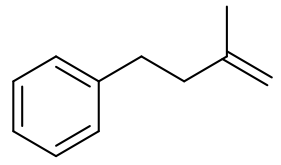

$$
1 \mathrm{x}
$$

${ }^{1} \mathrm{H}$ NMR, $400 \mathrm{MHz}, \mathrm{CDCl}_{3}$

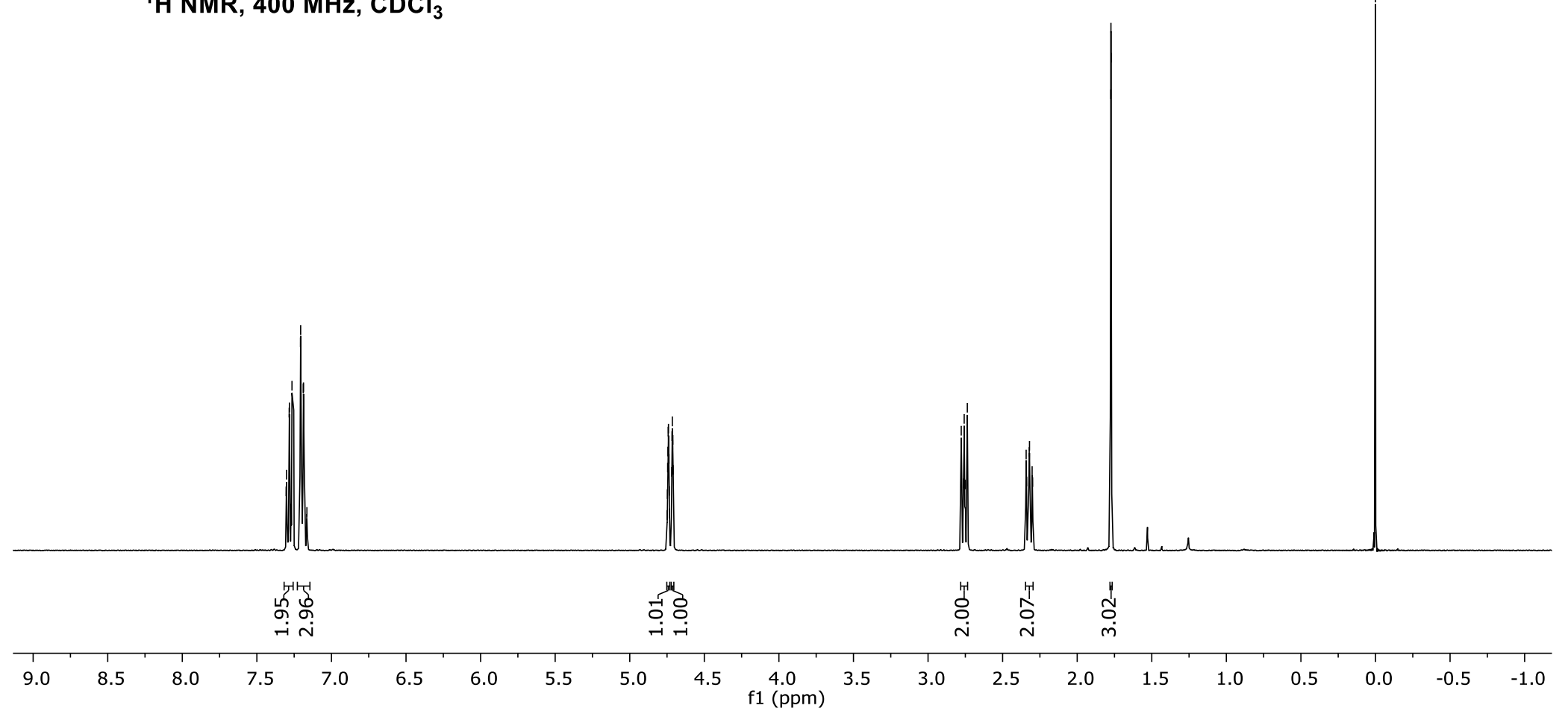


กำ

$\checkmark$

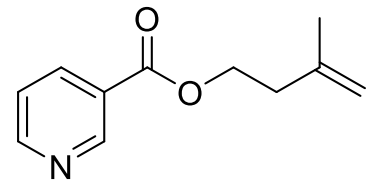

$1 y$

${ }^{1} \mathrm{H} \mathrm{NMR}, 400 \mathrm{MHz}, \mathrm{CDCl}_{3}$

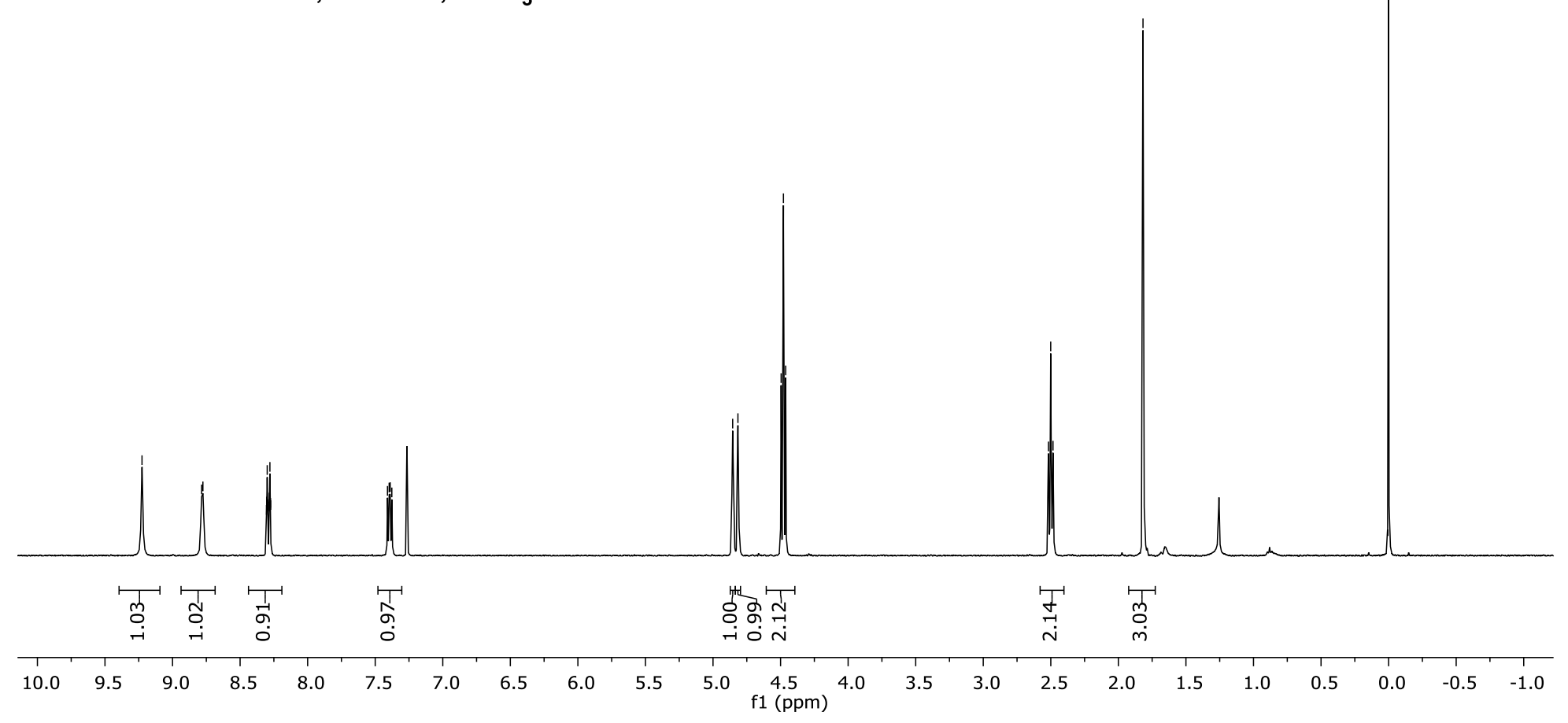




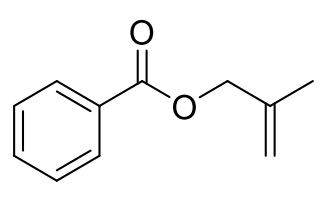

$1 z$

${ }^{1} \mathrm{H} \mathrm{NMR}, 500 \mathrm{MHz}, \mathrm{CDCl}_{3}$

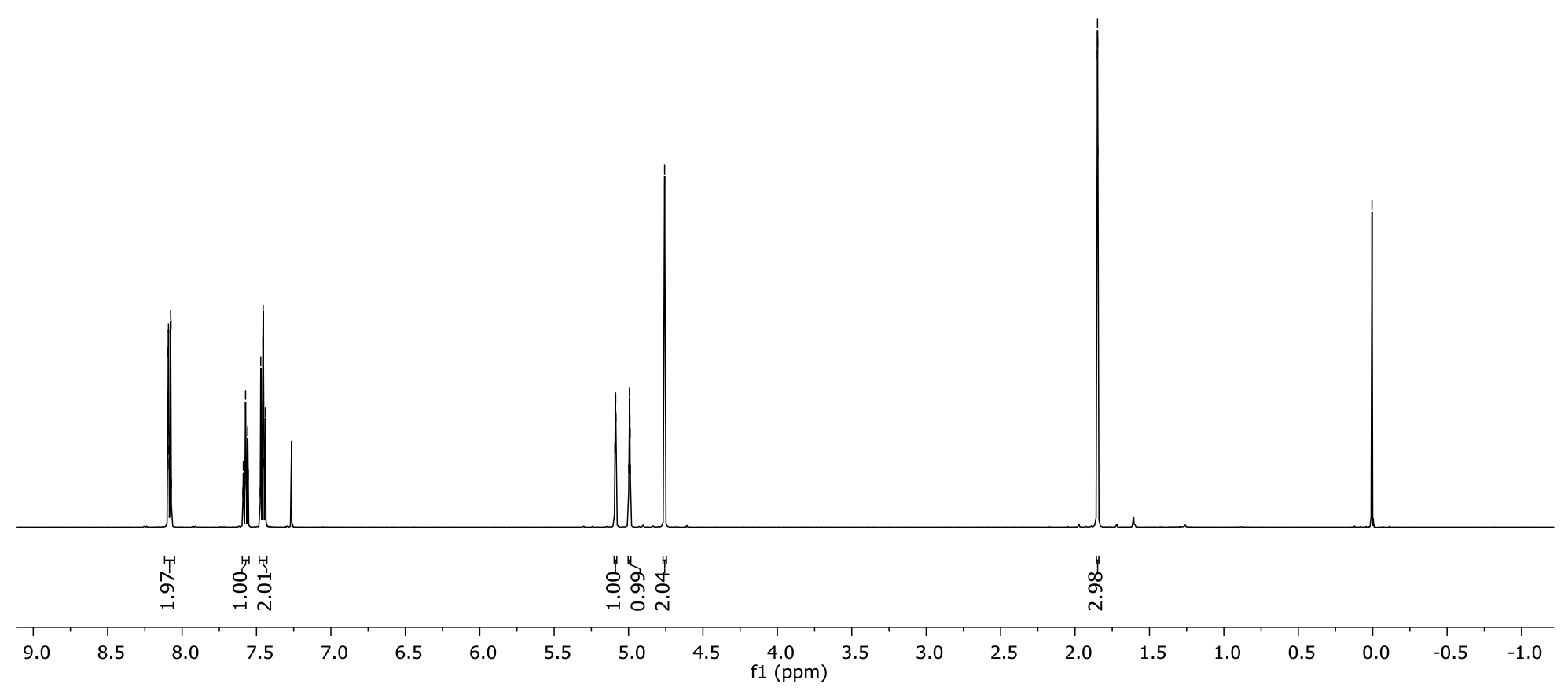


œ

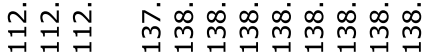

i

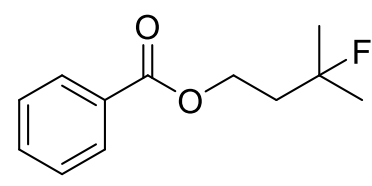

2a

${ }^{19} \mathrm{~F}$ NMR, $470 \mathrm{MHz}, \mathrm{CDCl}_{3}$

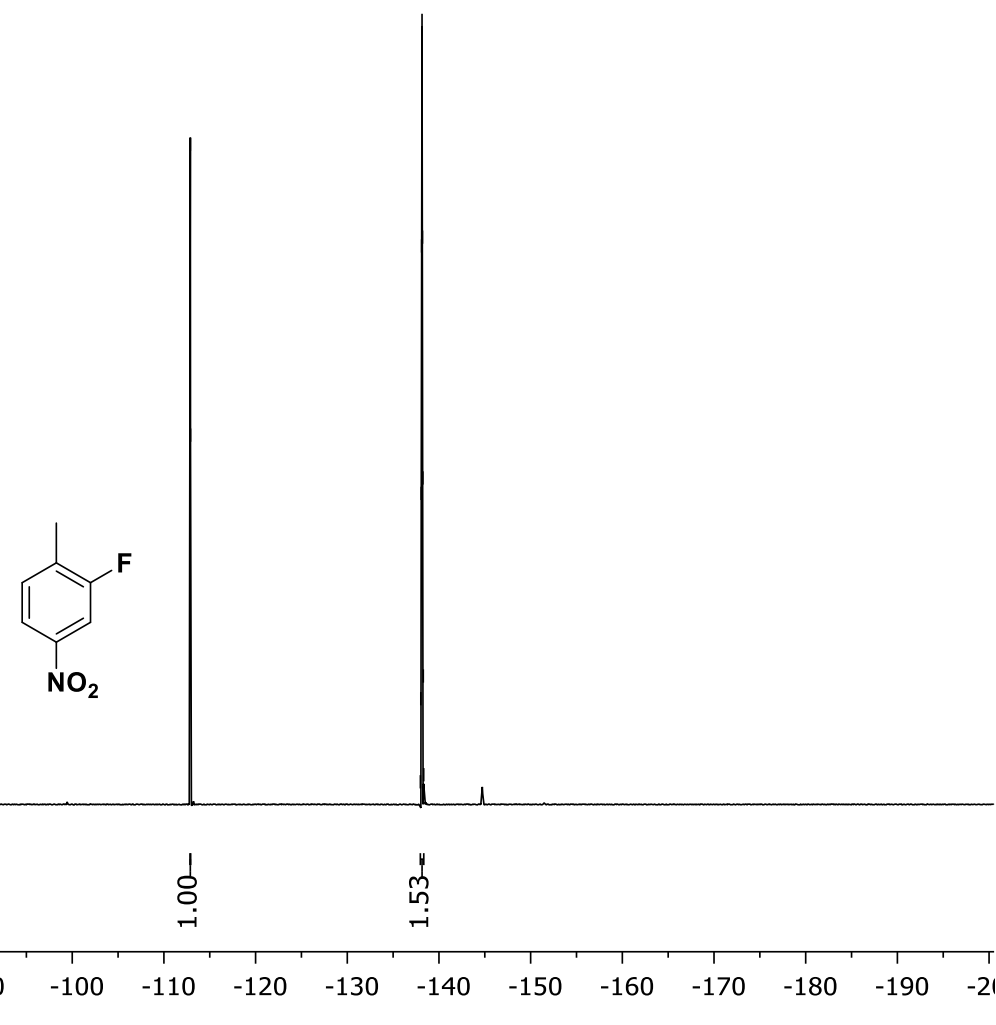




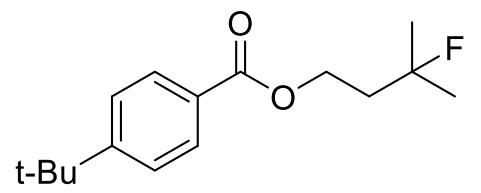

2b

${ }^{1} \mathrm{H}$ NMR, $500 \mathrm{MHz}, \mathrm{CDCl}_{3}$

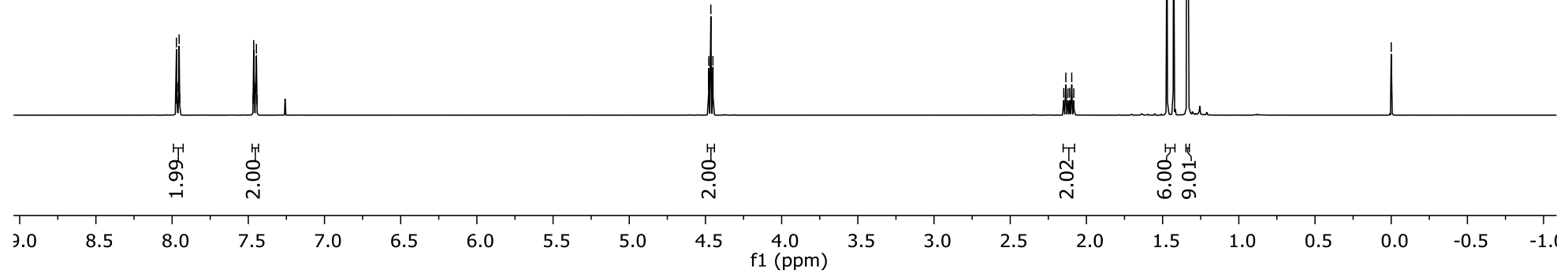




\begin{tabular}{|c|c|c|c|c|}
\hline 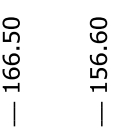 & 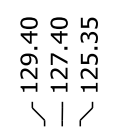 & $\begin{array}{l}\text { ज. oे } \\
\text { जु } \\
\text { ô } \\
11\end{array}$ & 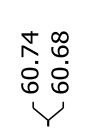 & 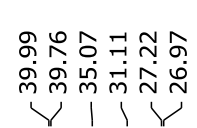 \\
\hline
\end{tabular}
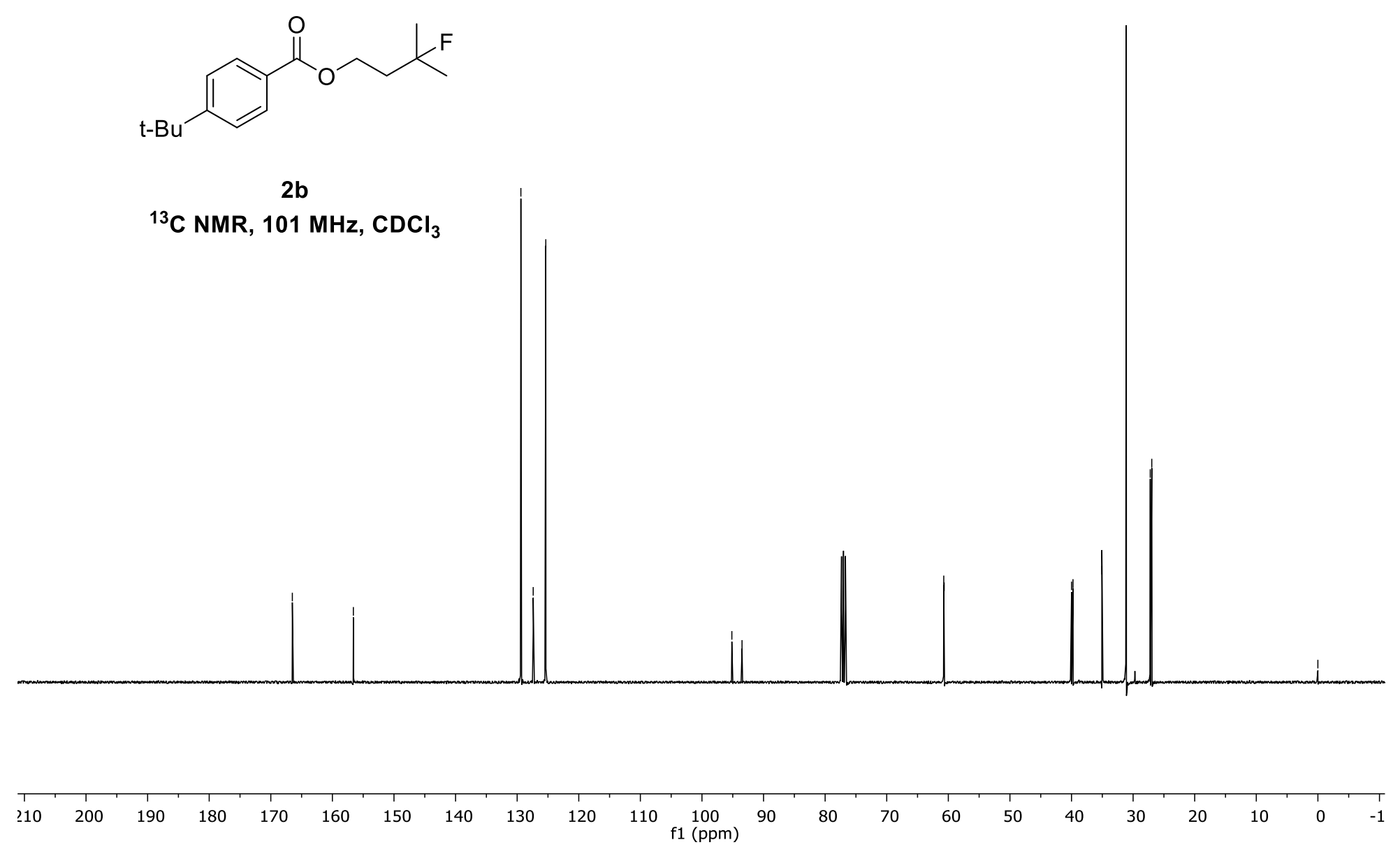


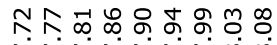

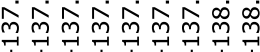

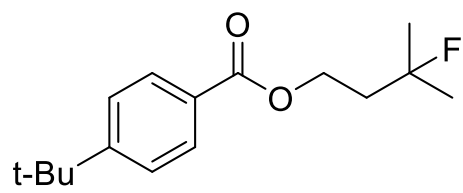

2b

${ }^{19} \mathrm{~F} \mathrm{NMR}, 470 \mathrm{MHz}, \mathrm{CDCl}_{3}$

\section{$\stackrel{d}{\circ}$}

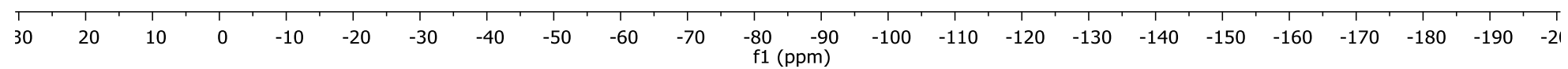




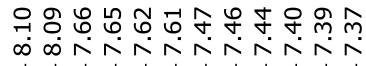

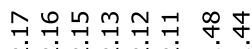

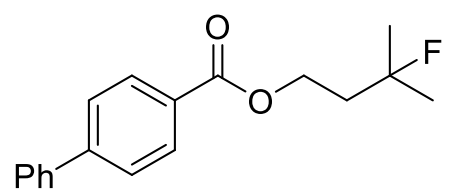

2c

${ }^{1} \mathrm{H}$ NMR, $500 \mathrm{MHz}, \mathrm{CDCl}_{3}$

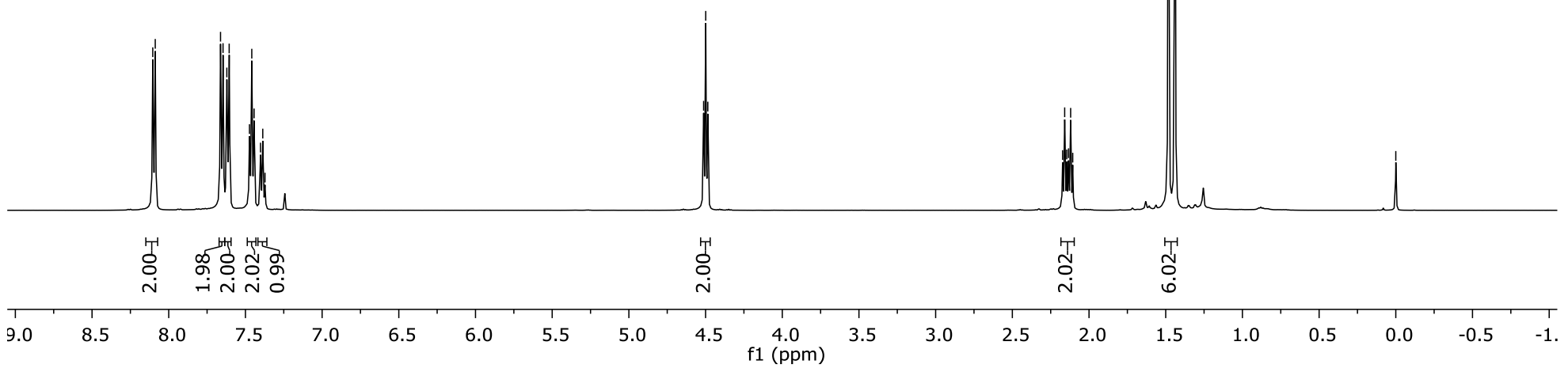




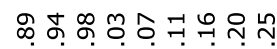

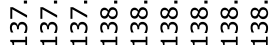

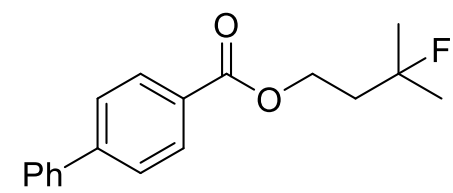

2c

${ }^{19} \mathrm{~F}$ NMR, $470 \mathrm{MHz}^{\mathrm{CDCl}_{3}}$

$\stackrel{\leftrightarrow}{\circ}$

\begin{tabular}{llllllllllllllllllllllllllll}
\hline 30 & 20 & 10 & 0 & -10 & -20 & -30 & -40 & -50 & -60 & -70 & -80 & -90 & -100 & -110 & -120 & -130 & -140 & -150 & -160 & -170 & -180 & -190 & -21
\end{tabular} 
<smiles>CC(C)(F)CCOC(=O)c1ccc([N+](=O)[O-])cc1</smiles>

2d

${ }^{1} \mathrm{H} \mathrm{NMR}, 500 \mathrm{MHz}, \mathrm{CDCl}_{3}$

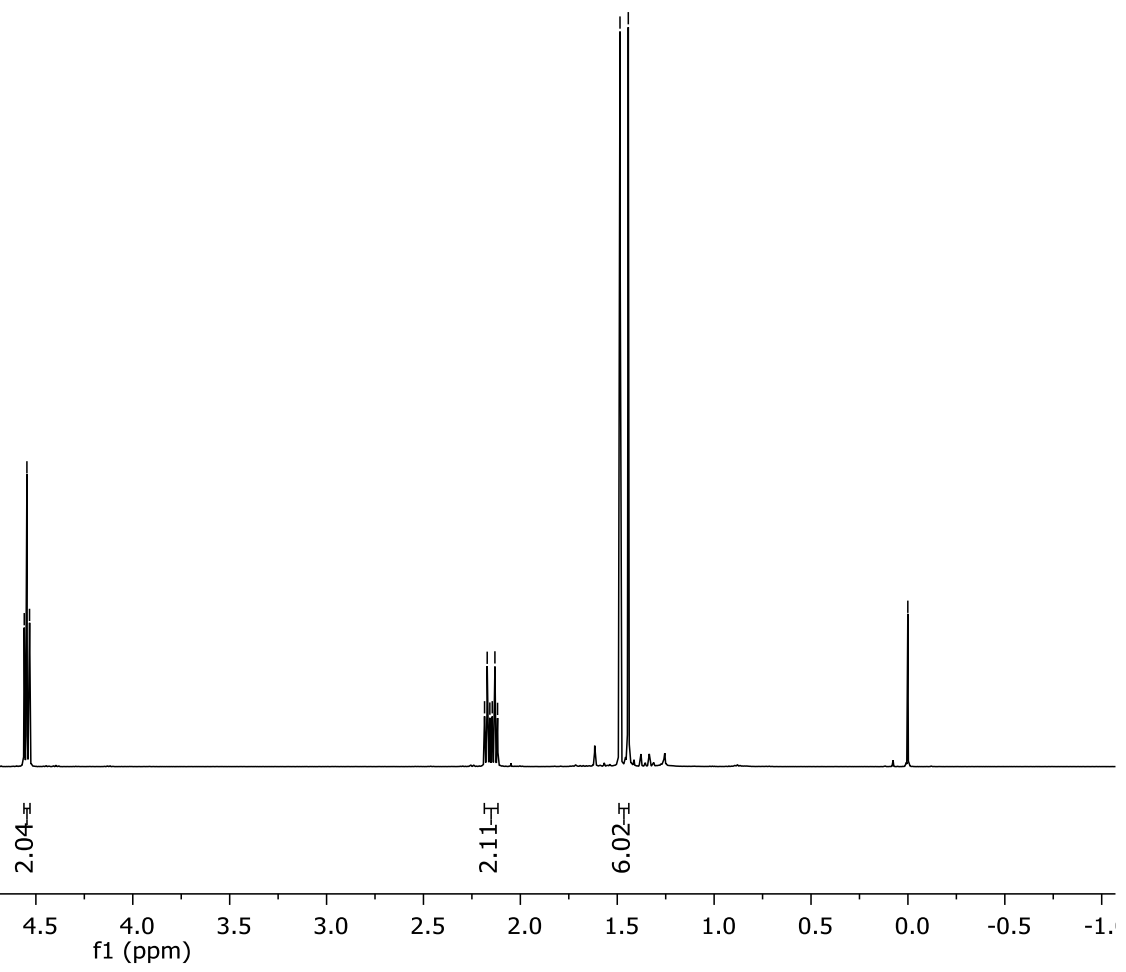




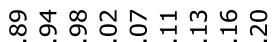

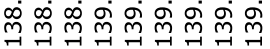

管

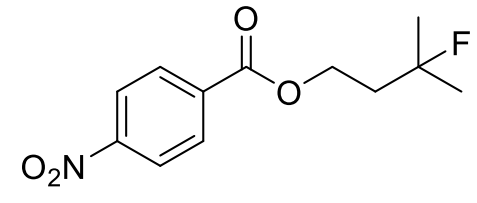

2d

${ }^{19} \mathrm{~F} \mathrm{NMR}, 500 \mathrm{MHz}, \mathrm{CDCl}_{3}$

\section{$\stackrel{\circ}{\circ}$}

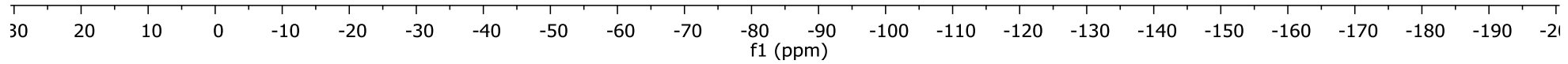




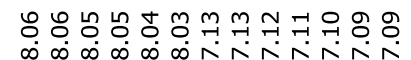

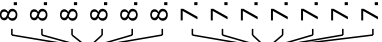

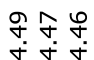

$\sqrt[i]{2+2+2}$

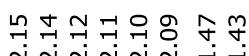

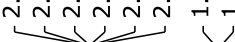

$\stackrel{0}{\circ}$

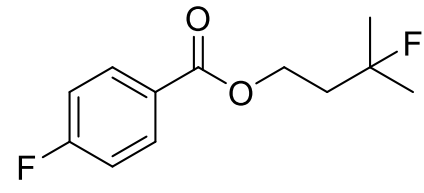

$2 e$

${ }^{1} \mathrm{H}$ NMR, $500 \mathrm{MHz}, \mathrm{CDCl}_{3}$

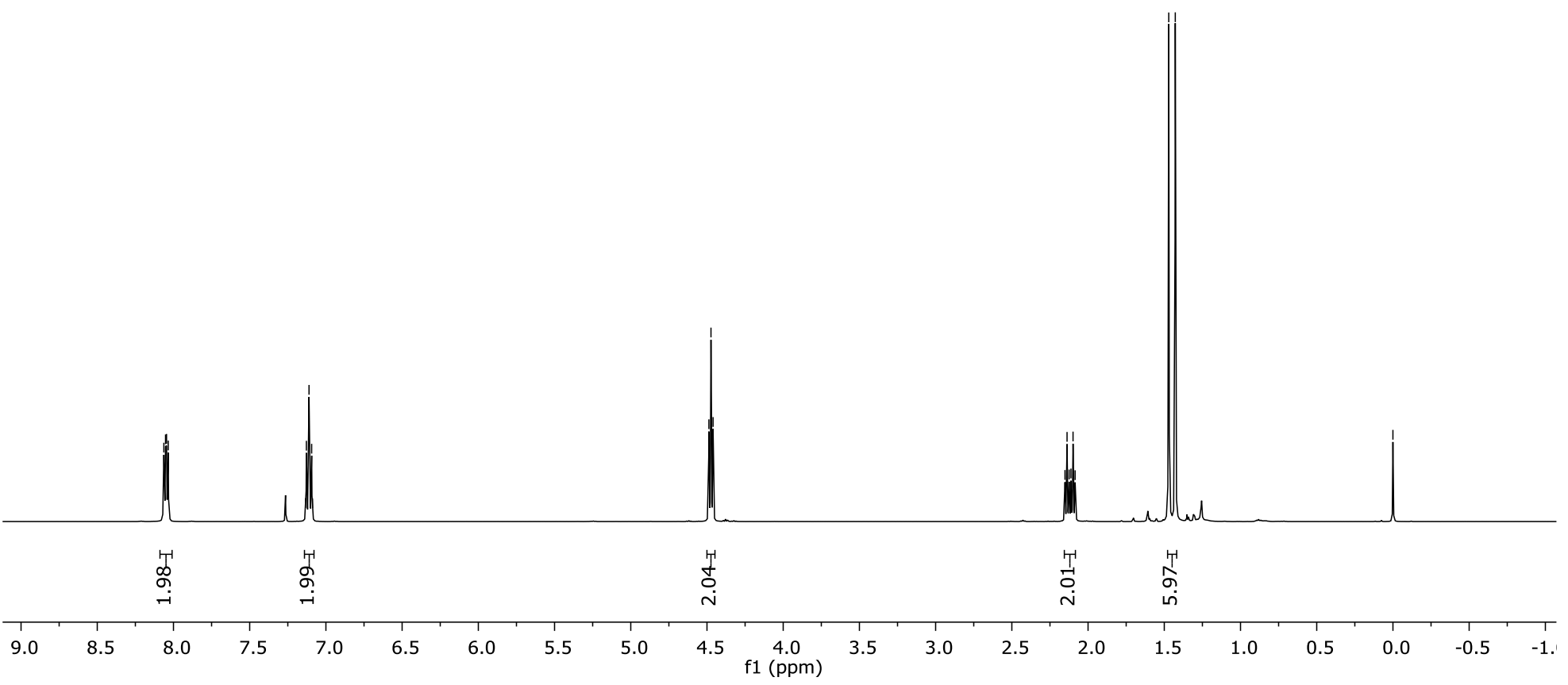




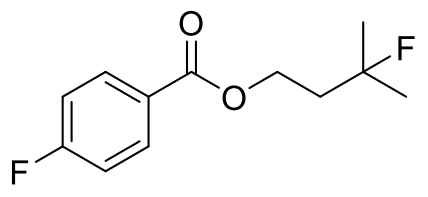

$2 e$

${ }^{19} \mathrm{~F} \mathrm{NMR}, 470 \mathrm{MHz}^{\mathrm{CDCl}}{ }_{3}$
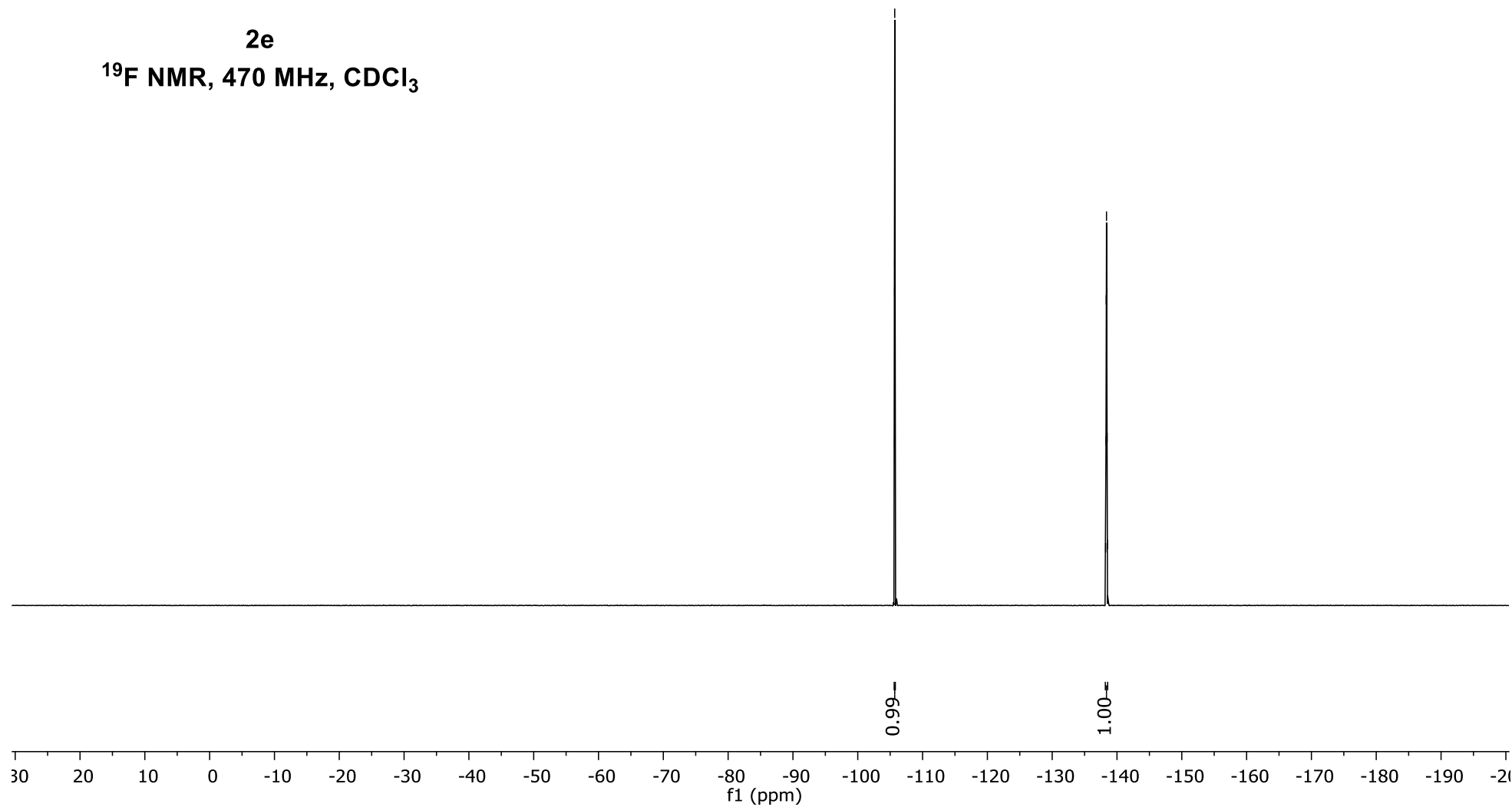


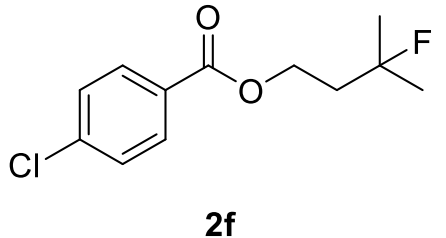

${ }^{1} \mathrm{H}$ NMR, $500 \mathrm{MHz}, \mathrm{CDCl}_{3}$

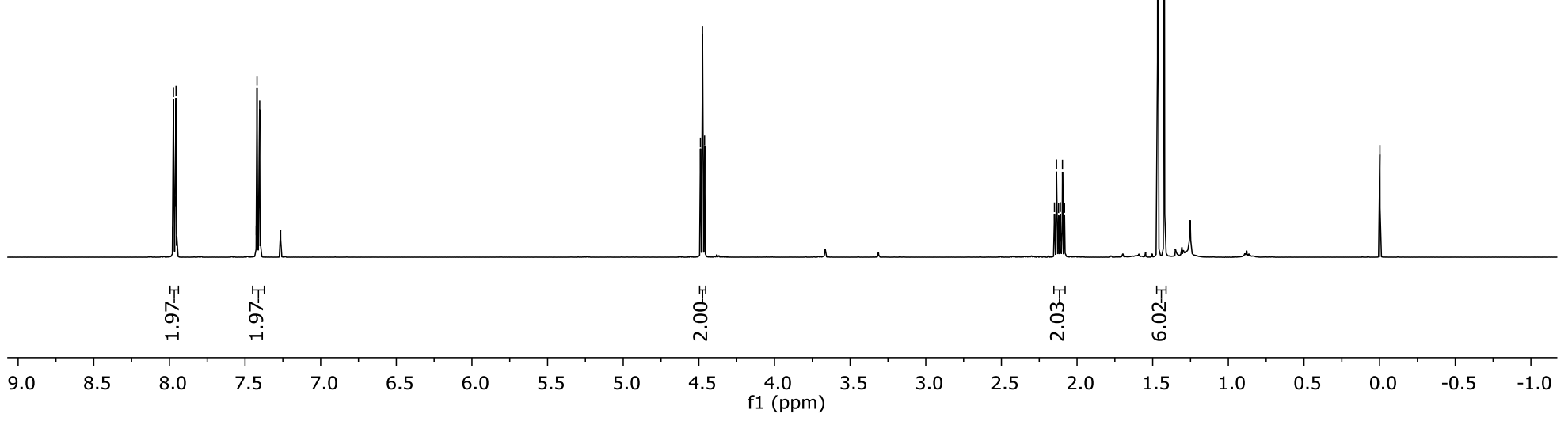




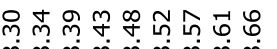

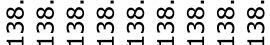

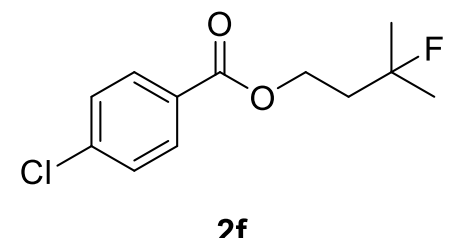

${ }^{19} \mathrm{~F} \mathrm{NMR,}, 470 \mathrm{MHz}, \mathrm{CDCl}_{3}$

$\stackrel{\circ}{\circ}$

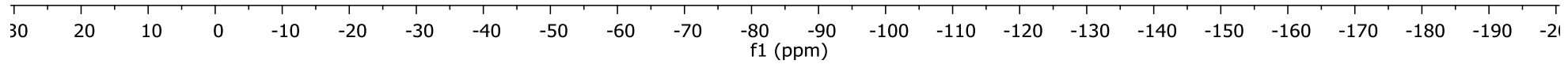




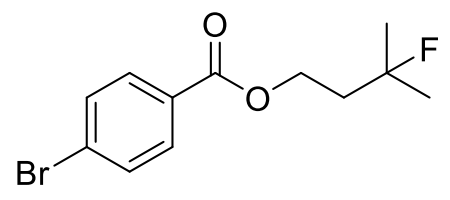

$2 \mathrm{~g}$

${ }^{1} \mathrm{H} \mathrm{NMR}, 500 \mathrm{MHz}, \mathrm{CDCl}_{3}$

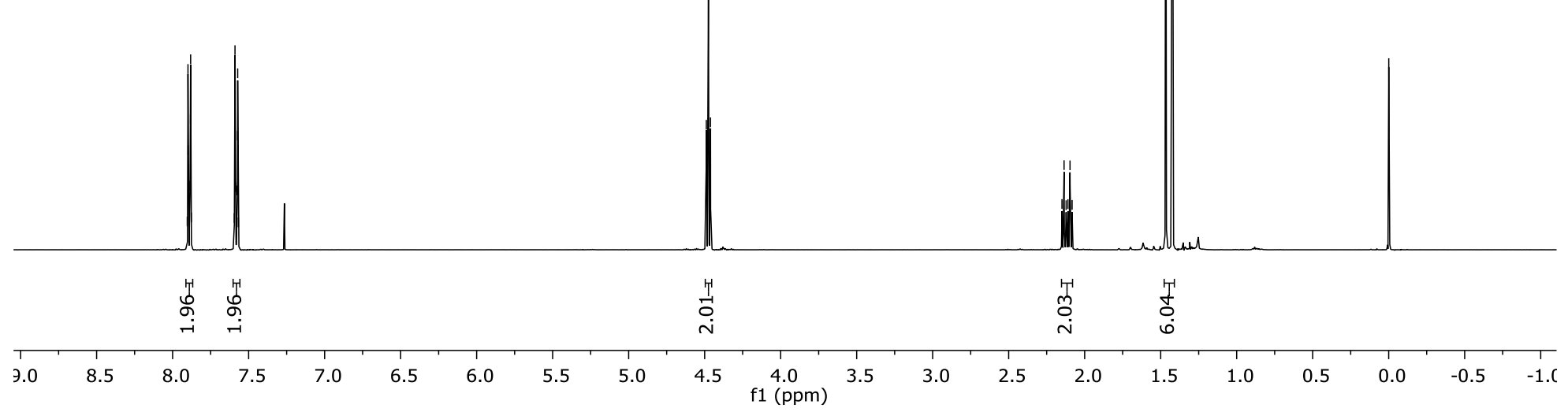




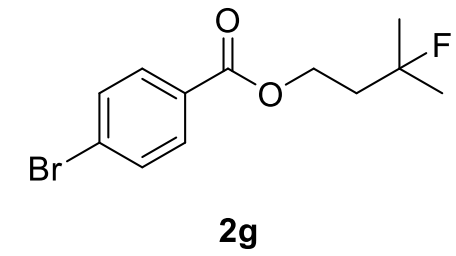

${ }^{13} \mathrm{C} \mathrm{NMR}, 101 \mathrm{MHz}^{\mathrm{CDCl}}{ }_{3}$ 


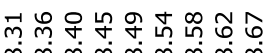

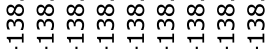

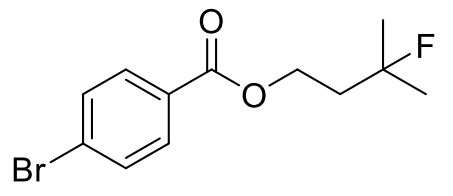

$2 \mathrm{~g}$

${ }^{19} \mathrm{~F}$ NMR, $470 \mathrm{MHz}, \mathrm{CDCl}_{3}$

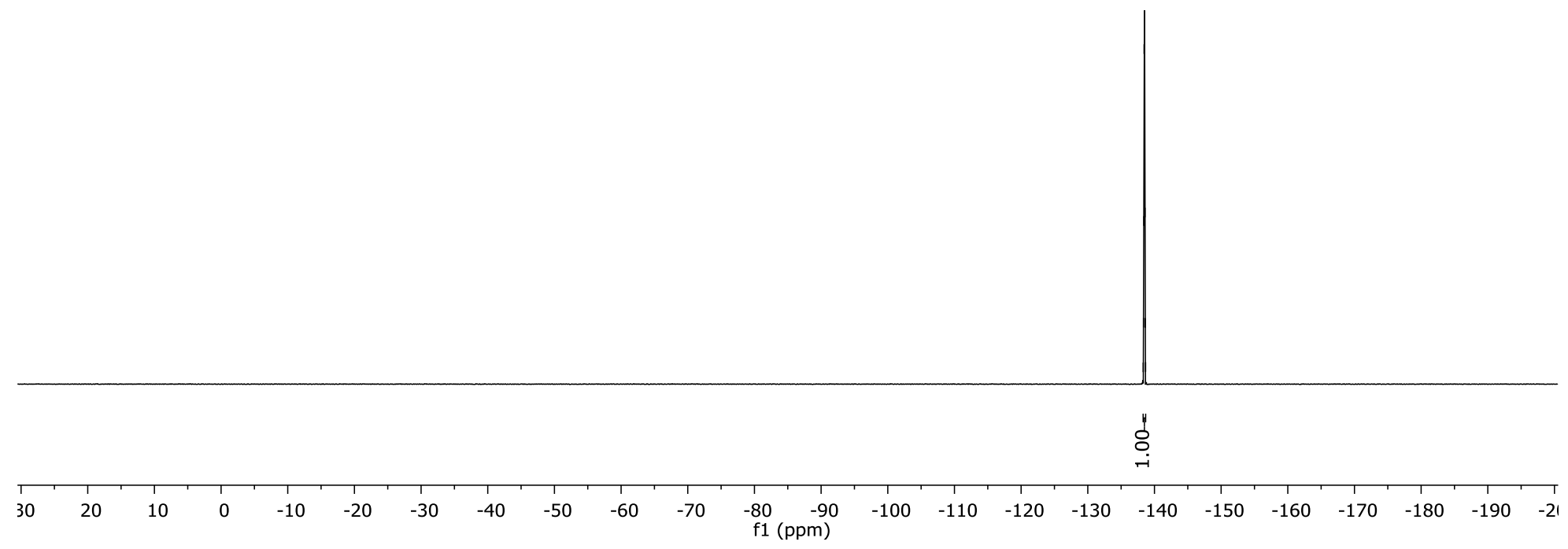




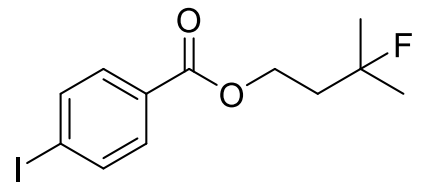

2h

${ }^{1} \mathrm{H}$ NMR, $470 \mathrm{MHz}, \mathrm{CDCl}_{3}$

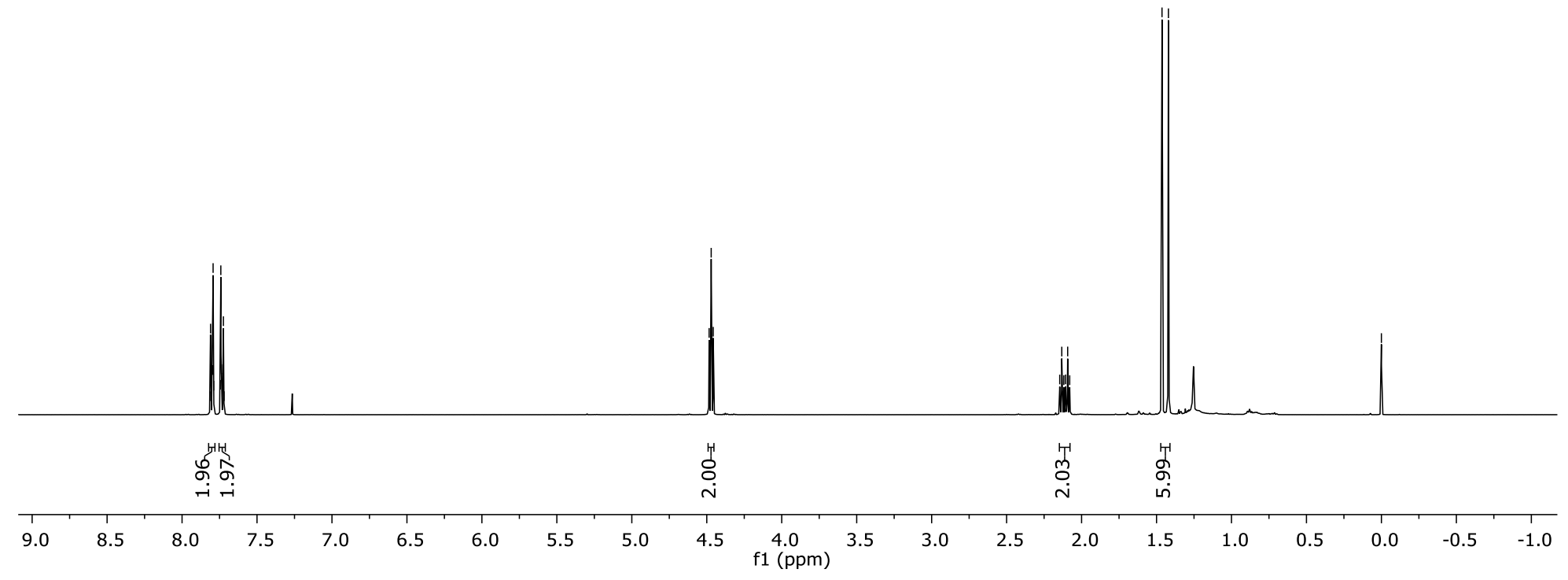




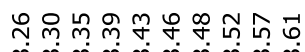

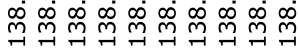

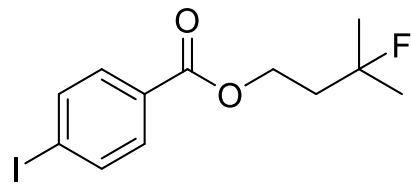

$2 \mathrm{~h}$

${ }^{19} \mathrm{~F} \mathrm{NMR}, 470 \mathrm{MHz}, \mathrm{CDCl}_{3}$

\section{$\stackrel{4}{\circ}$}

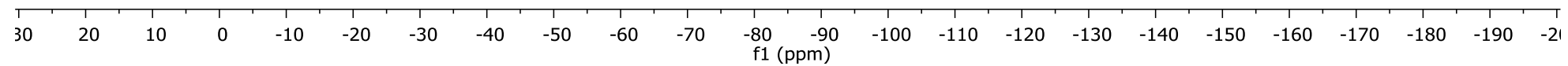




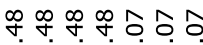

$\underbrace{\infty} \underbrace{\infty} \underbrace{\infty}$

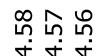

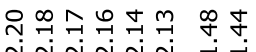

$\underbrace{0 . \mu j}$

$\stackrel{8}{\circ}$<smiles>CC(C)(F)CCOC(=O)c1cc(C(F)(F)F)cc(C(F)(F)F)c1</smiles>

$2 \mathrm{i}$

${ }^{1} \mathrm{H}$ NMR, $500 \mathrm{MHz}, \mathrm{CDCl}_{3}$

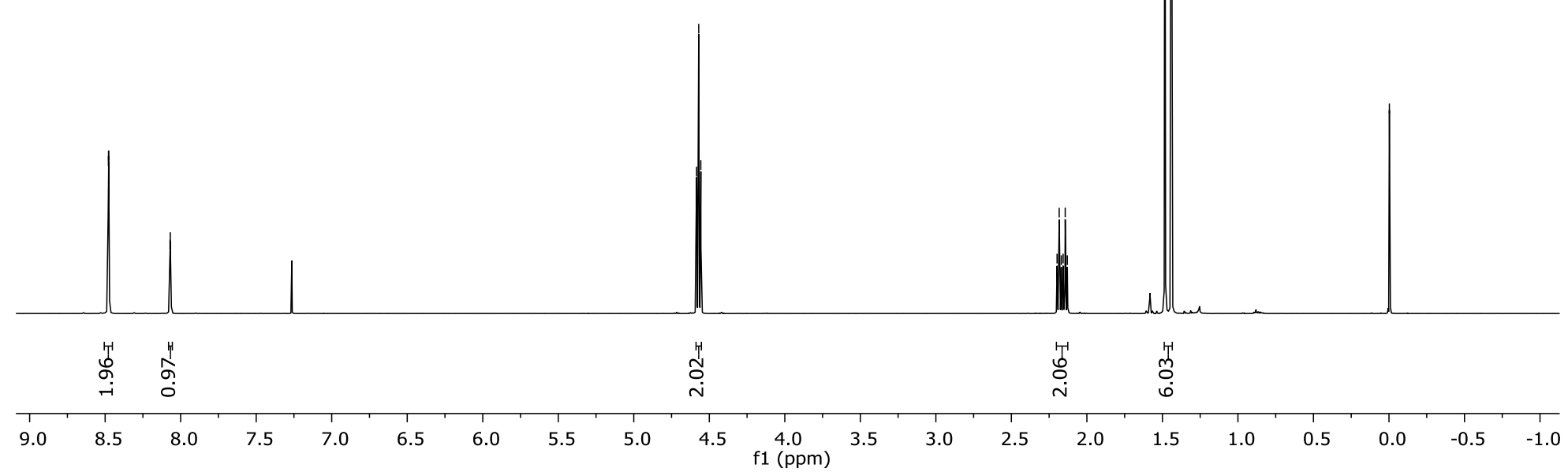


<smiles>CC(C)(F)CCOC(=O)c1cc(C(F)(F)F)cc(C(F)(F)F)c1</smiles>

2i

${ }^{13} \mathrm{C}$ NMR, $101 \mathrm{MHz}, \mathrm{CDCl}_{3}$ 


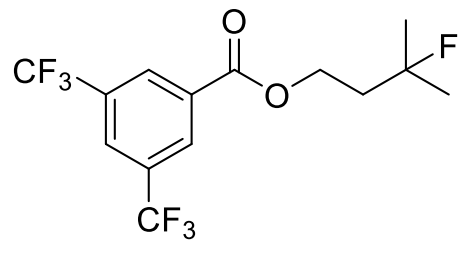

2i

${ }^{19} \mathrm{~F}$ NMR, $470 \mathrm{MHz}^{\mathrm{CDCl}} \mathrm{CD}_{3}$

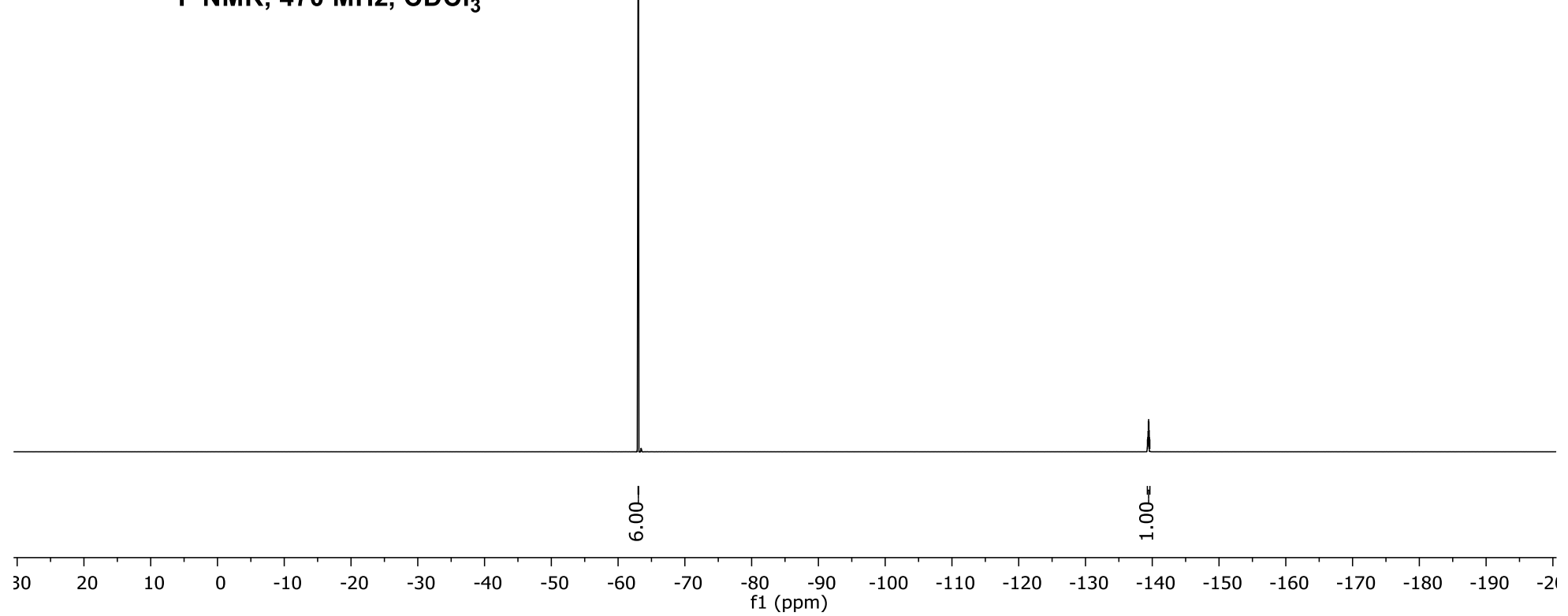




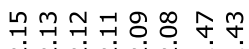

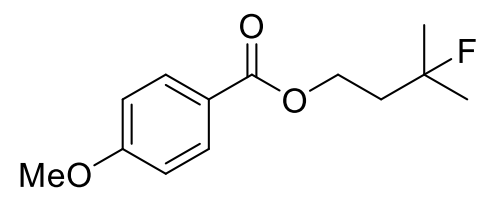

2j

${ }^{1} \mathrm{H} \mathrm{NMR}, 500 \mathrm{MHz}, \mathrm{CDCl}_{3}$

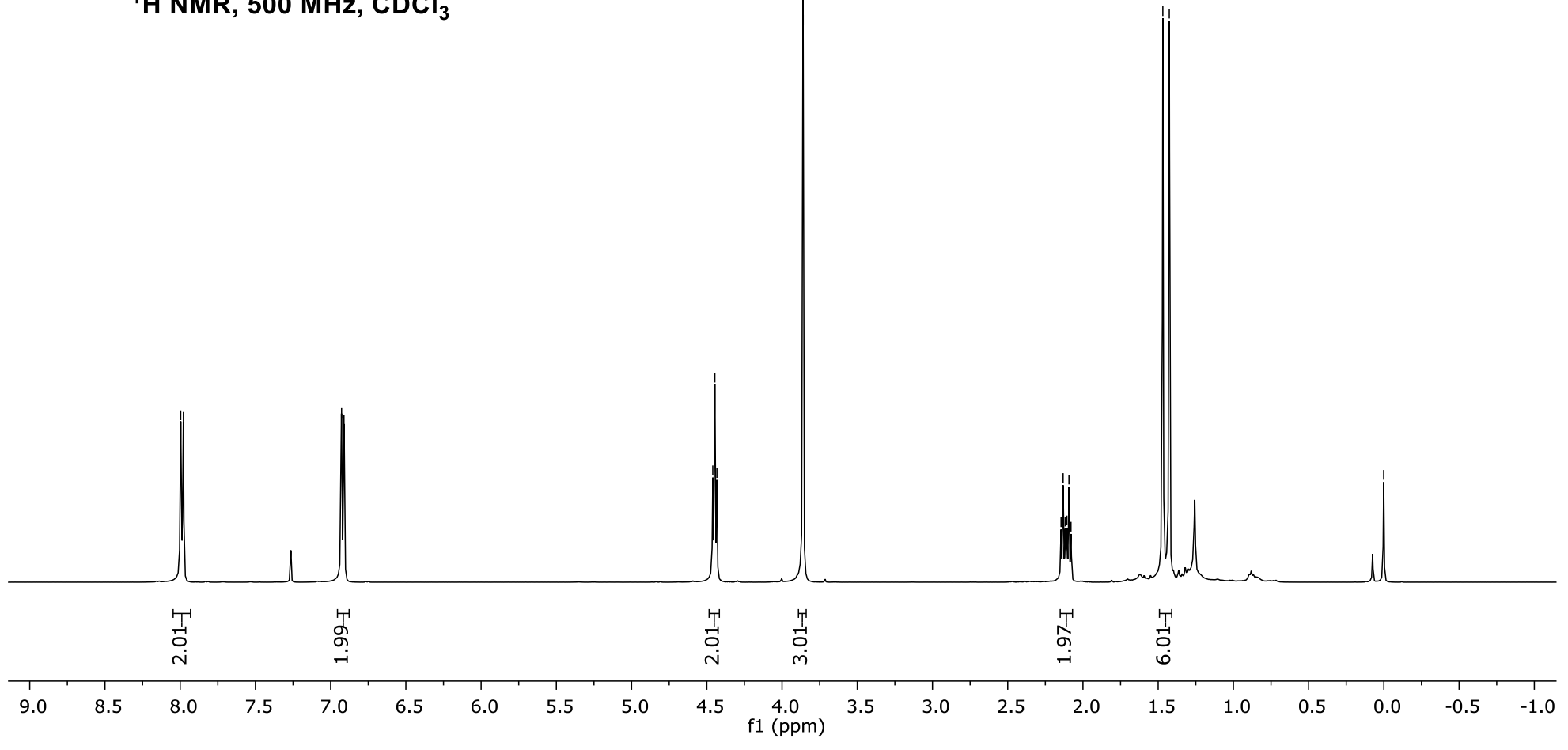




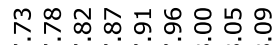

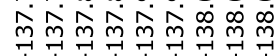

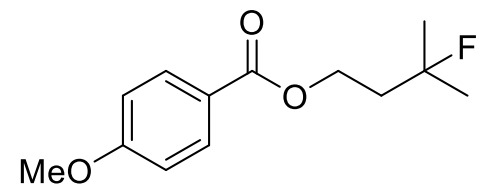

2j

${ }^{19} \mathrm{~F} \mathrm{NMR}, 470 \mathrm{MHz}, \mathrm{CDCl}_{3}$

\section{$\stackrel{1}{\circ}$}

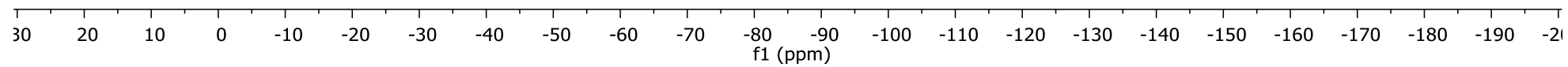




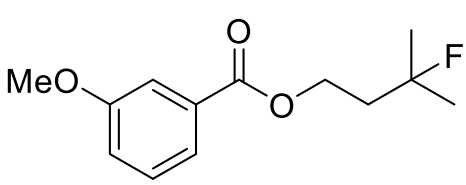

2k

${ }^{1} \mathrm{H}$ NMR, $500 \mathrm{MHz}, \mathrm{CDCl}_{3}$

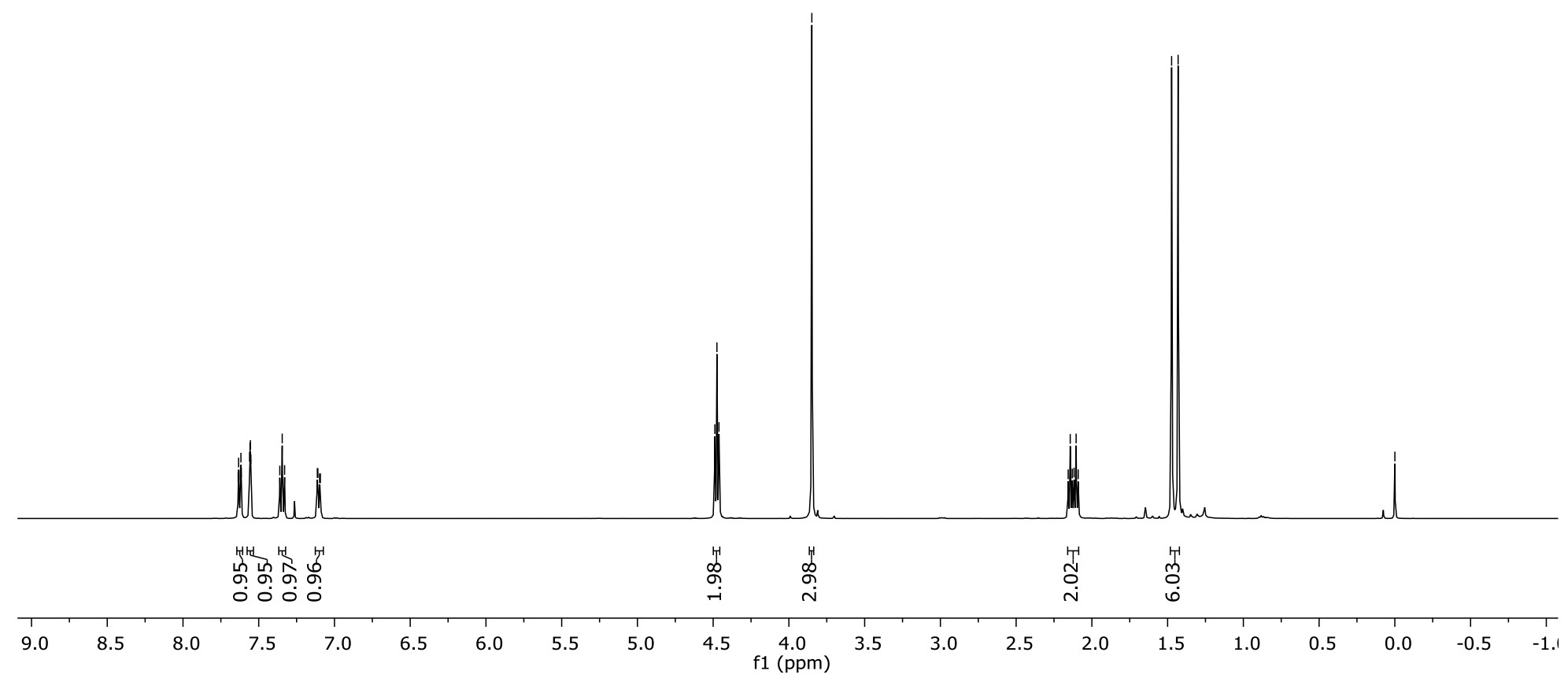


ดㅎำ

$\hat{m}$

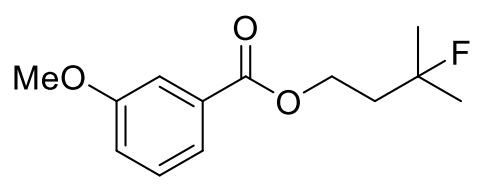

2k

${ }^{19} \mathrm{~F} \mathrm{NMR}, 470 \mathrm{MHz}, \mathrm{CDCl}_{3}$

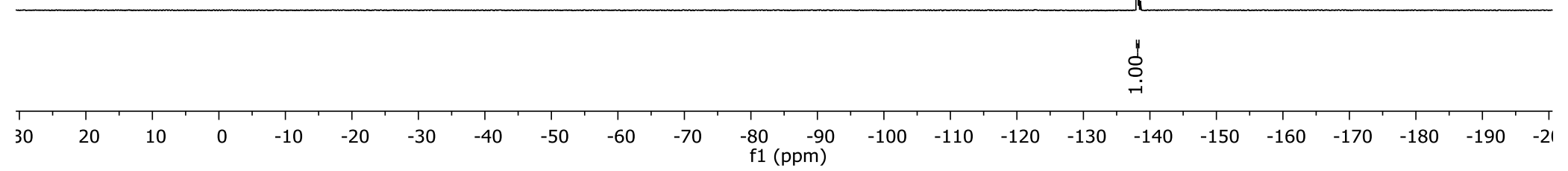




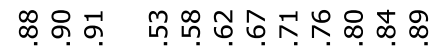

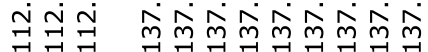

贷

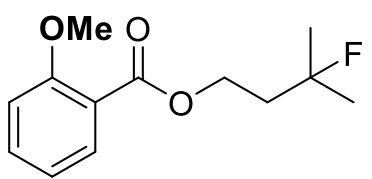

2I

${ }^{19} \mathrm{~F} \mathrm{NMR,}, 470 \mathrm{MHz}, \mathrm{CDCl}_{3}$

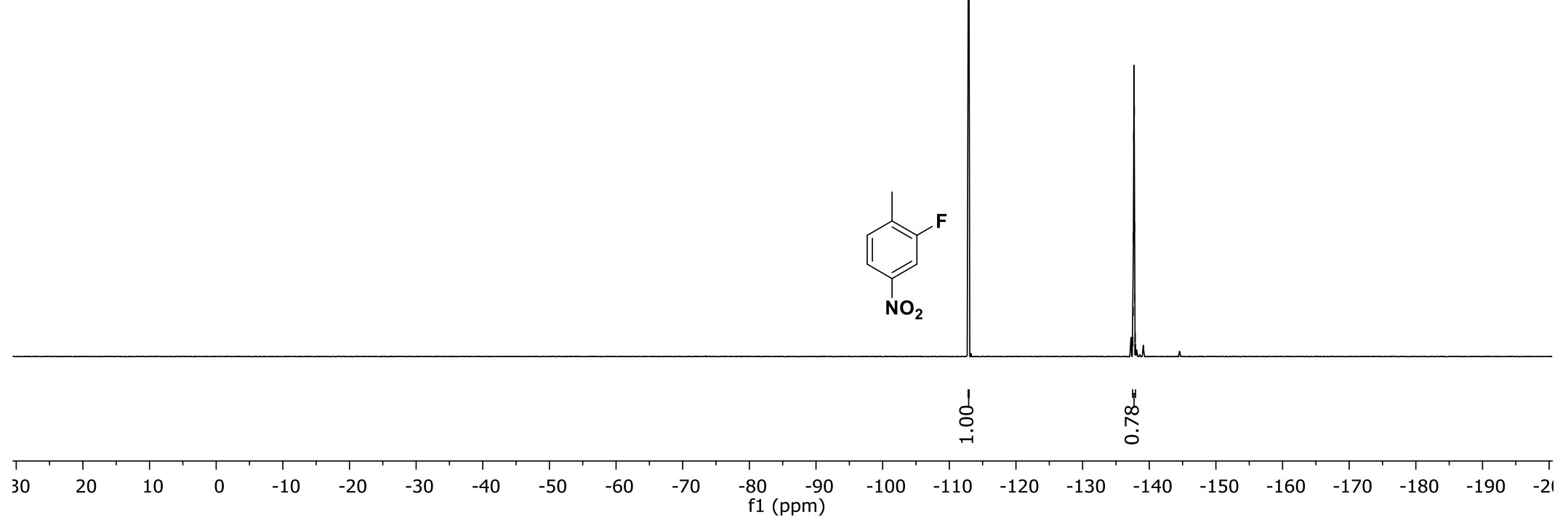


年 굿ำ

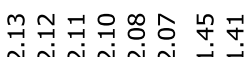

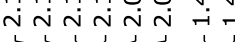

is

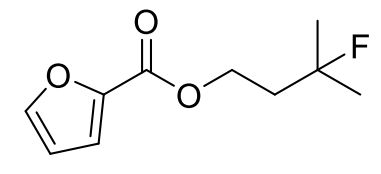

$2 \mathrm{~m}$

${ }^{1} \mathrm{H}$ NMR, $500 \mathrm{MHz}, \mathrm{CDCl}_{3}$

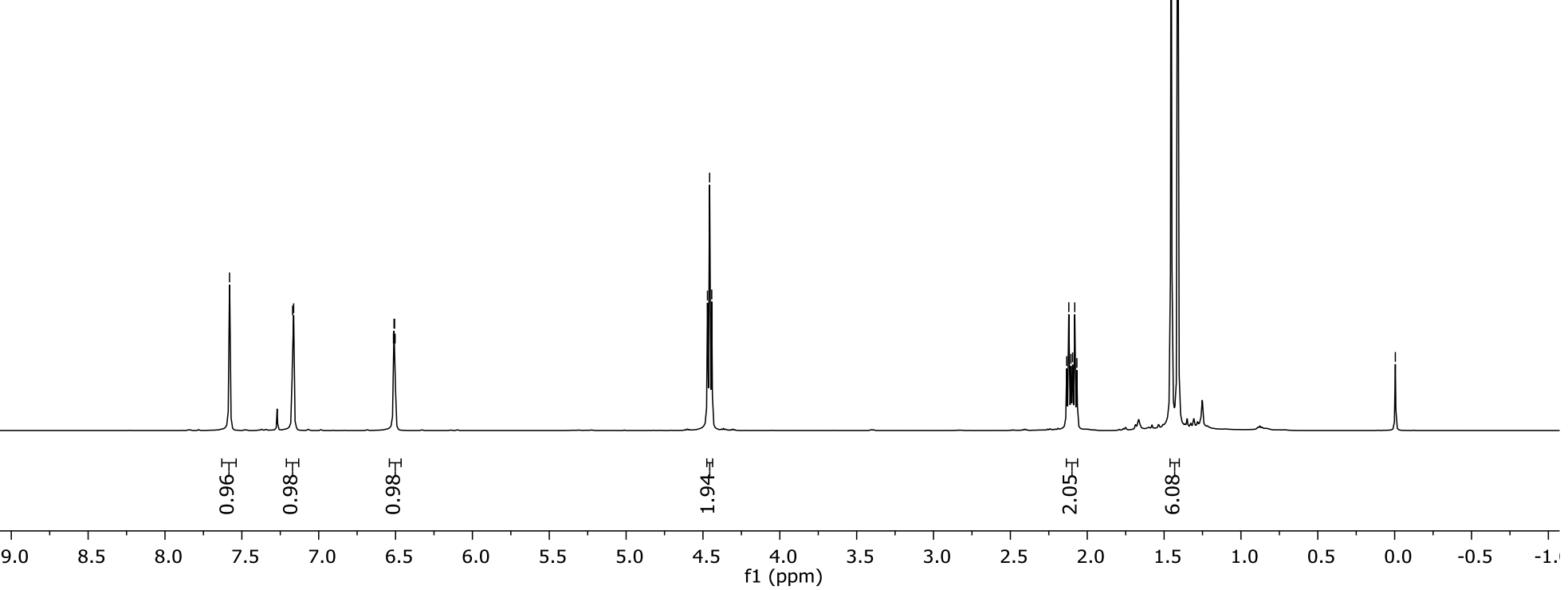




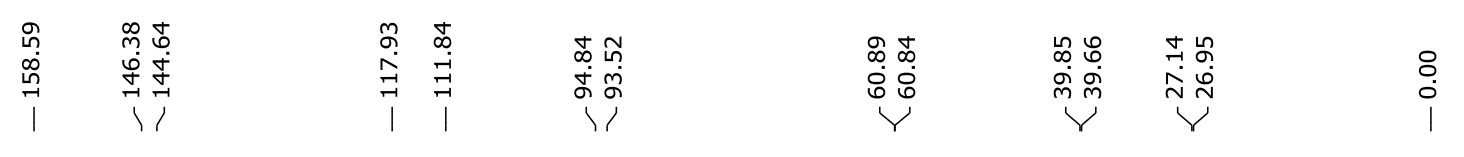

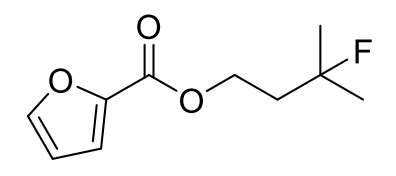

$2 \mathrm{~m}$

${ }^{13} \mathrm{C} \mathrm{NMR,} 126 \mathrm{MHz}, \mathrm{CDCl}_{3}$

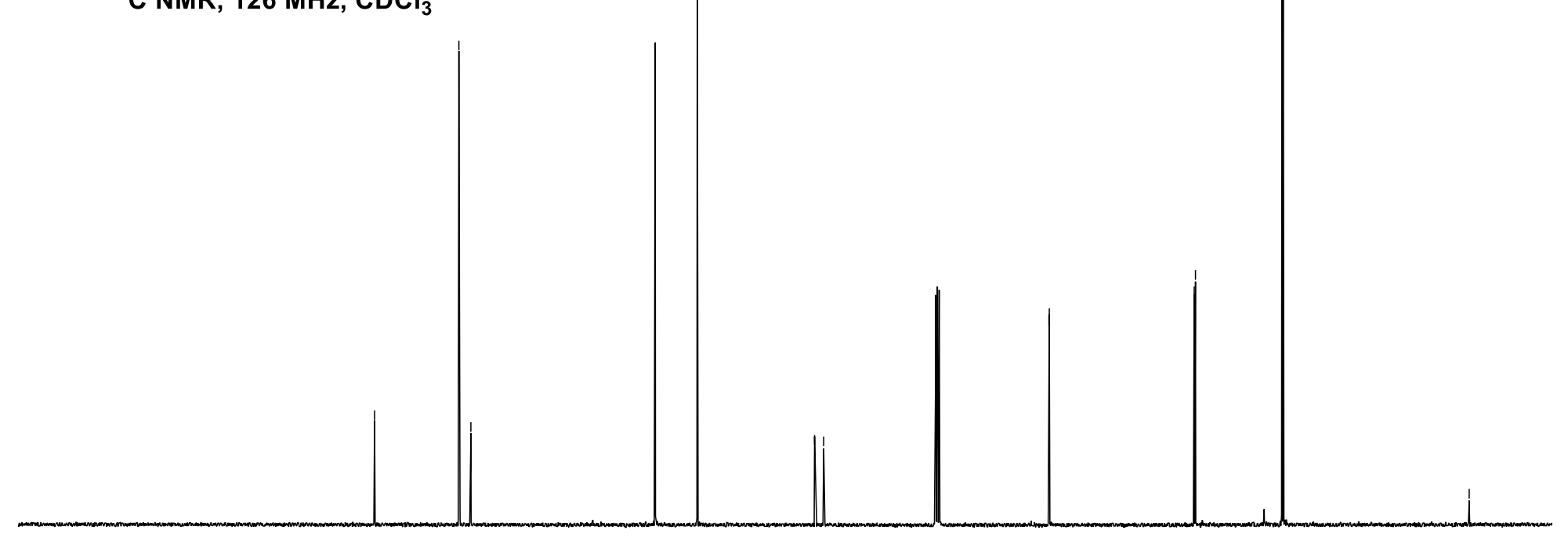

\begin{tabular}{lllllllllllllllllllllll}
\hline 10 & 200 & 190 & 180 & 170 & 160 & 150 & 140 & 130 & 120 & 110 & $\begin{array}{c}100 \\
\mathrm{f} 1(\mathrm{ppm})\end{array}$ & 90 & 80 & 70 & 60 & 50 & 40 & 30 & 20 & 10 & 0 & $-1 \mathrm{C}$
\end{tabular} 


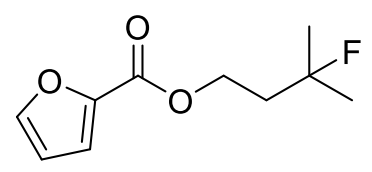

$2 \mathrm{~m}$

${ }^{19} \mathrm{~F}$ NMR, $470 \mathrm{MHz}, \mathrm{CDCl}_{3}$
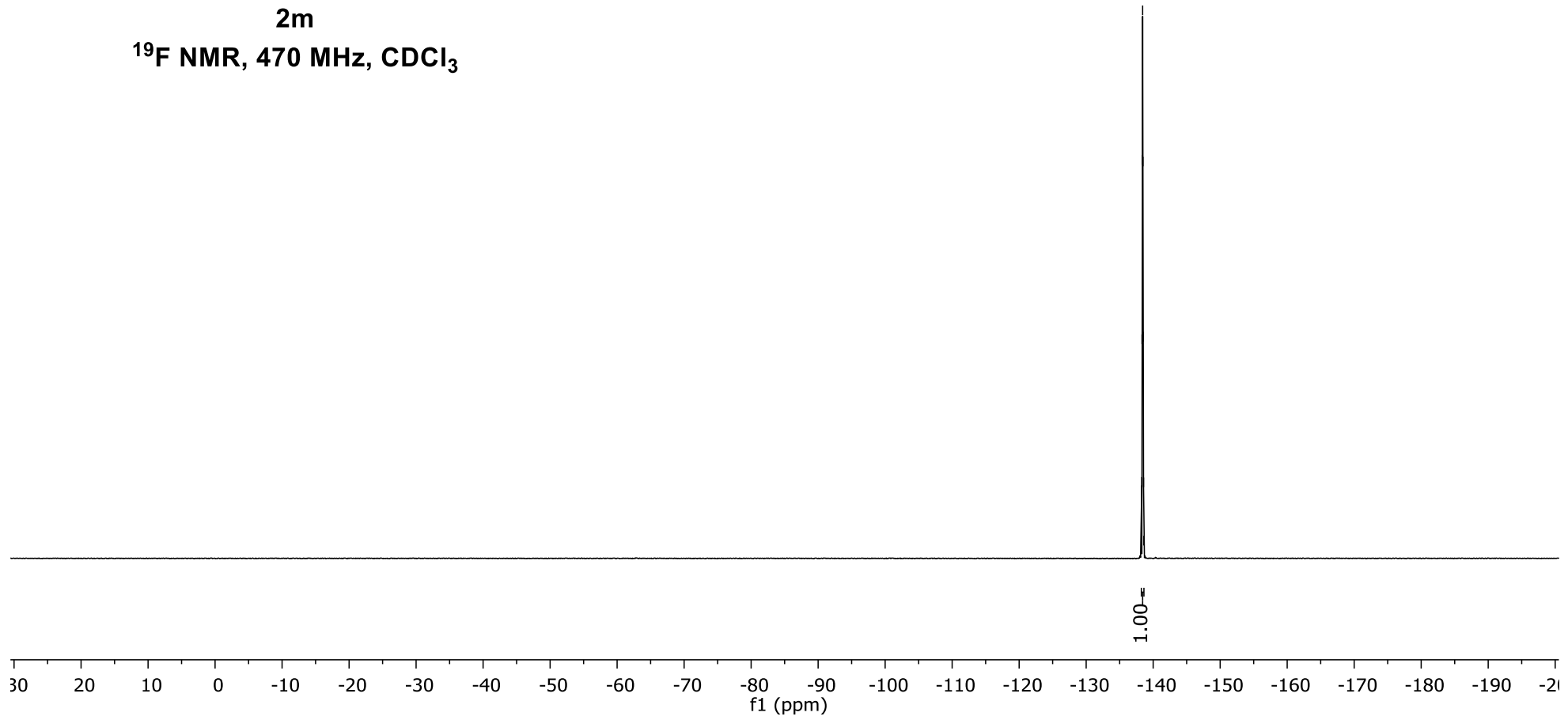


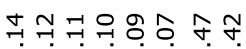

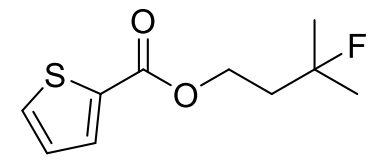

2n

${ }^{1} \mathrm{H}$ NMR, $500 \mathrm{MHz}, \mathrm{CDCl}_{3}$

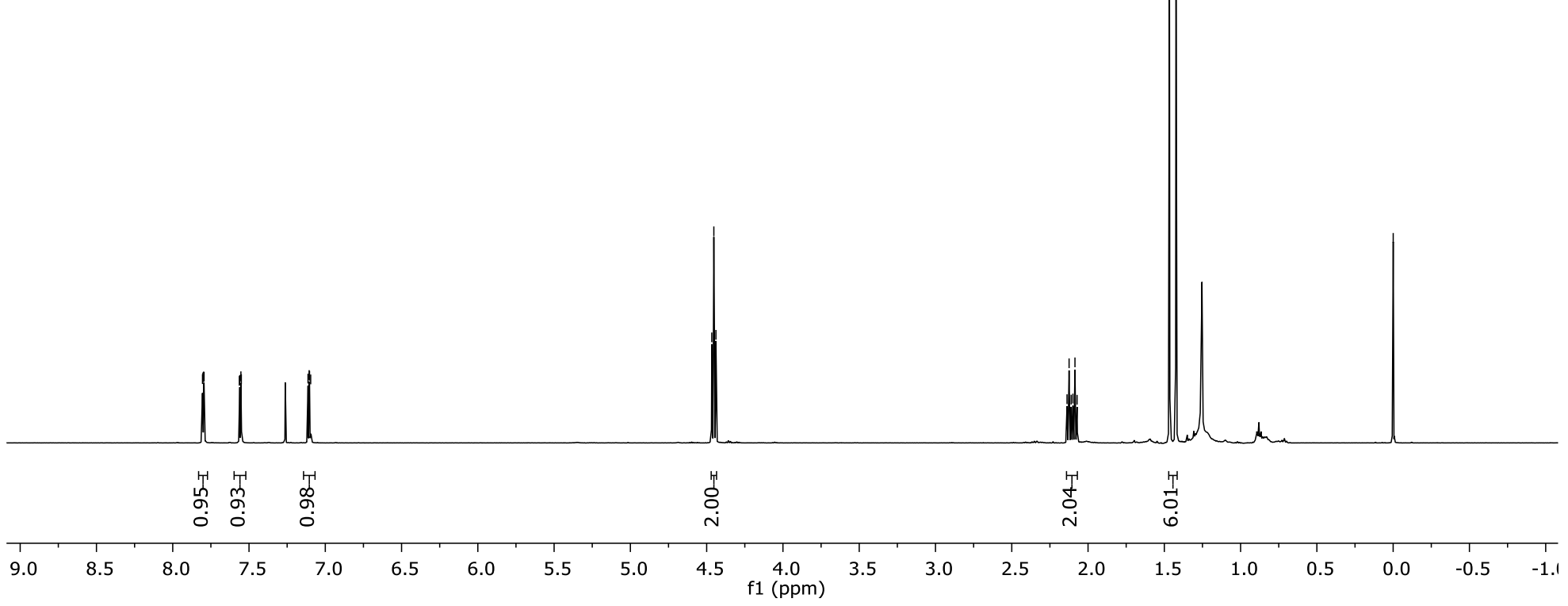




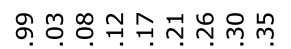

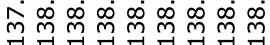

(1)

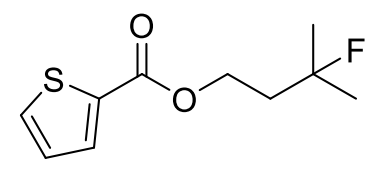

2n

${ }^{19} \mathrm{~F}$ NMR, $470 \mathrm{MHz}^{\mathrm{CDCl}}{ }_{3}$

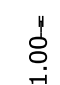

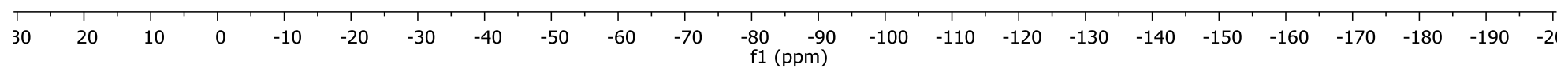




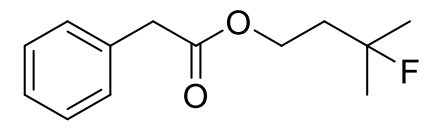

20

${ }^{1} \mathrm{H}$ NMR, $400 \mathrm{MHz}, \mathrm{CDCl}_{3}$

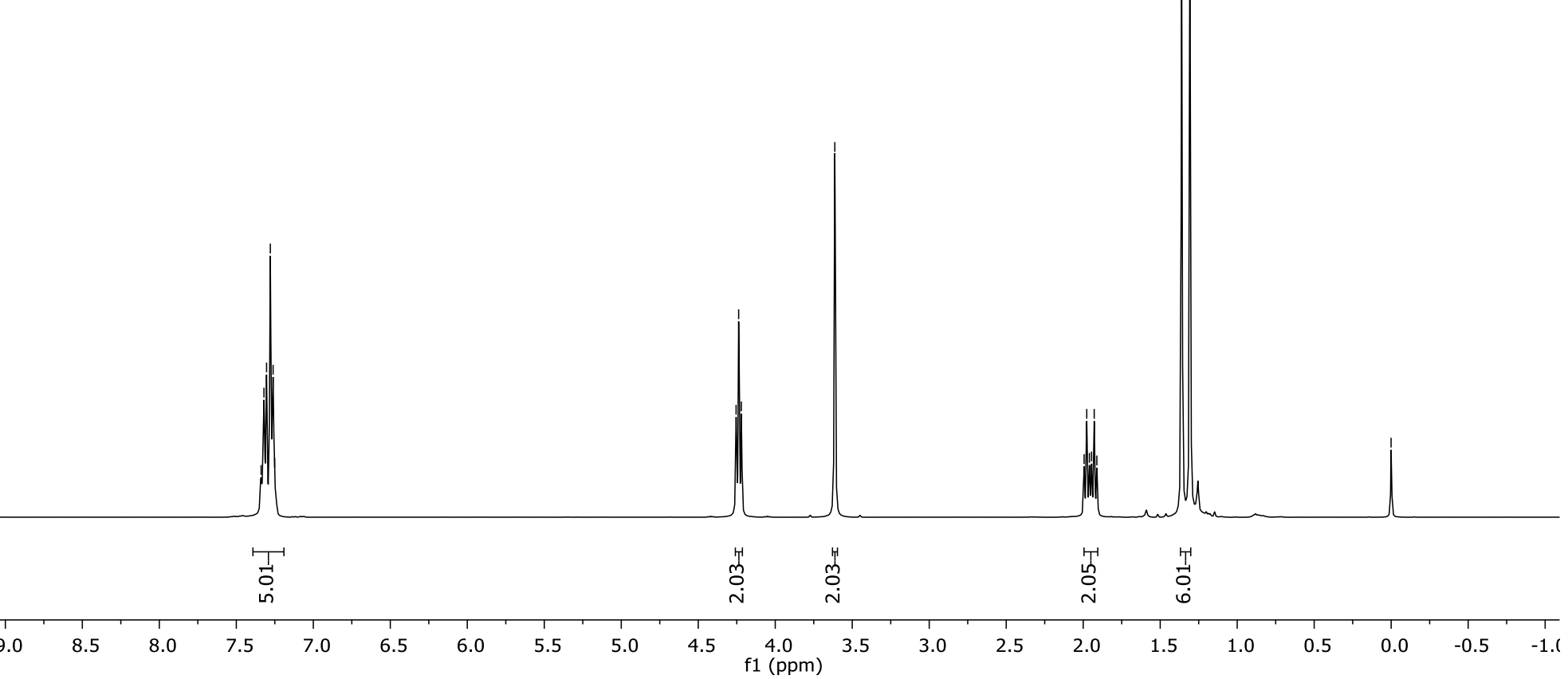




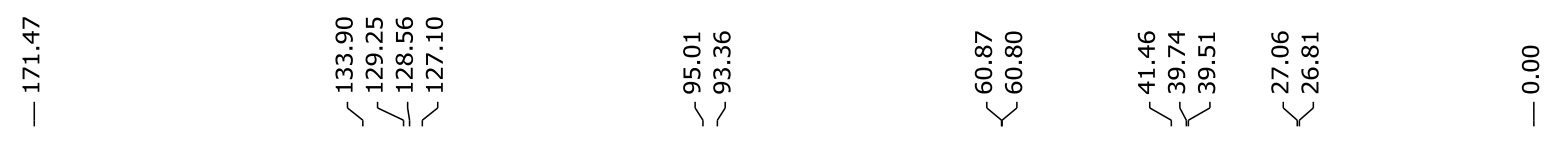

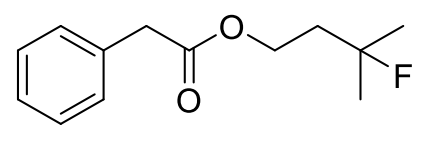

20

${ }^{13} \mathrm{C}$ NMR, $101 \mathrm{MHz}, \mathrm{CDCl}_{3}$

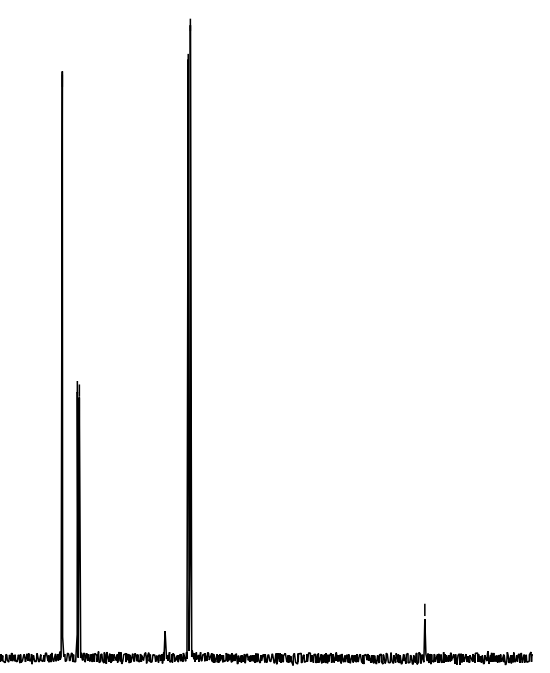

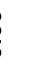




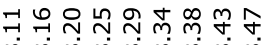

$\prod_{m}^{\infty} \prod^{\infty} \prod^{\infty} \infty \prod^{\infty} \infty m^{\infty} \infty m^{\infty} \infty$

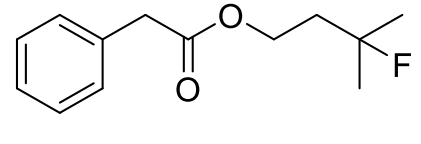

20

${ }^{19} \mathrm{~F} \mathrm{NMR}, 470 \mathrm{MHz}, \mathrm{CDCl}_{3}$

\section{$\stackrel{8}{-1}$}

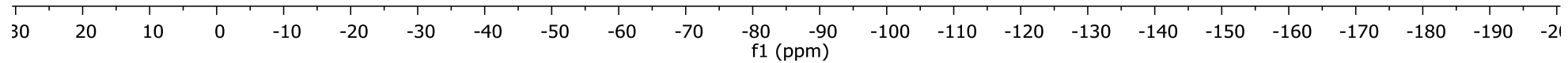




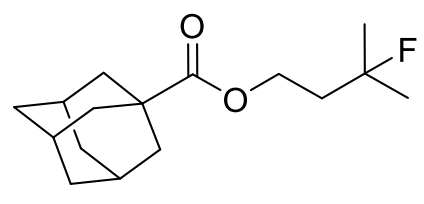

$2 p$

${ }^{1} \mathrm{H}$ NMR, $500 \mathrm{MHz}, \mathrm{CDCl}_{3}$

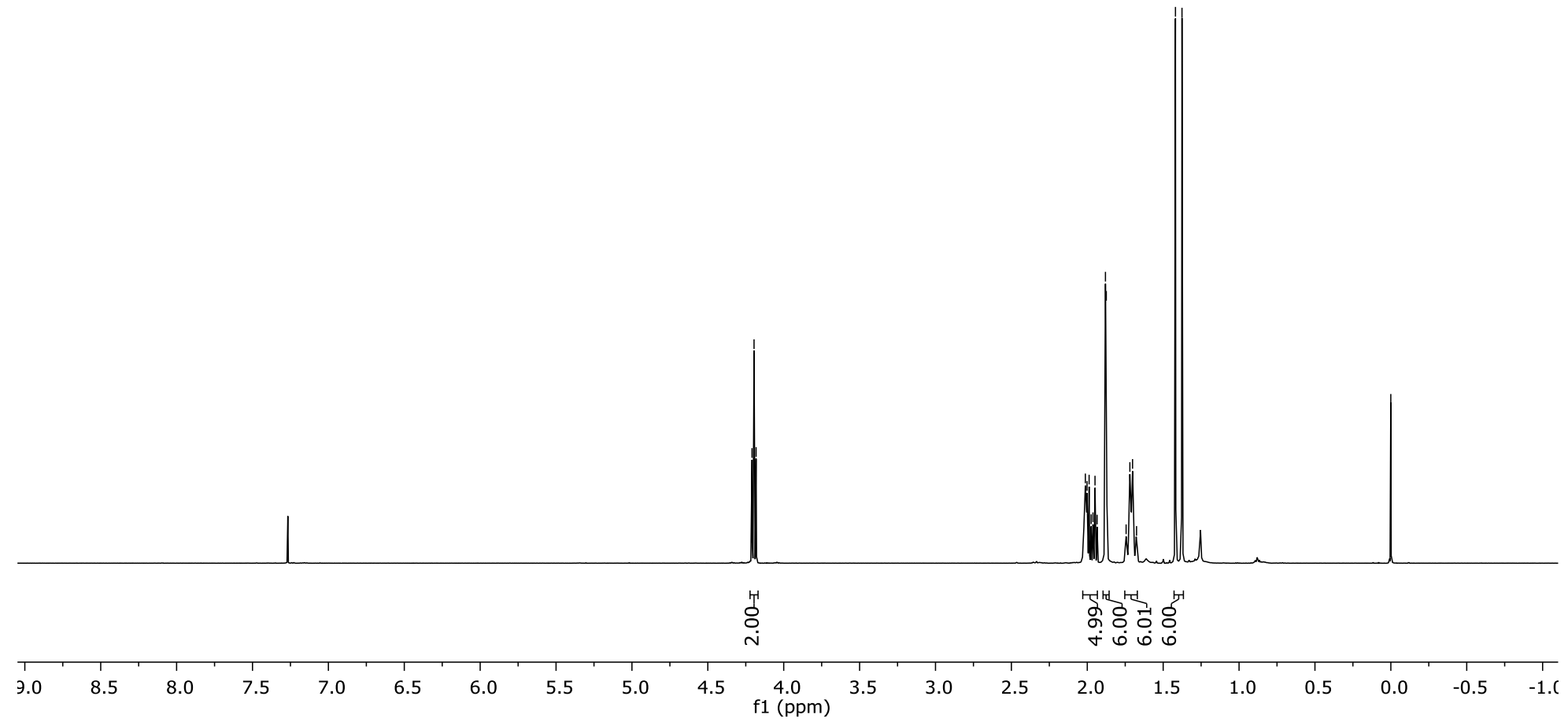




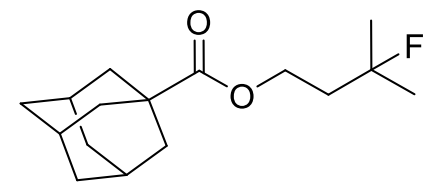

$2 p$

${ }^{13} \mathrm{C}$ NMR, $101 \mathrm{MHz}, \mathrm{CDCl}_{3}$

$10 \quad 200 \quad 190 \quad 180$

$180 \quad 17$

160

$150 \quad 140$

130

$120 \quad 110$

$110 \quad 100$

90

$70 \quad 60$

50

30

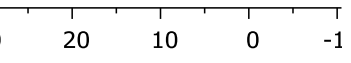


ํำ

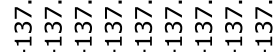

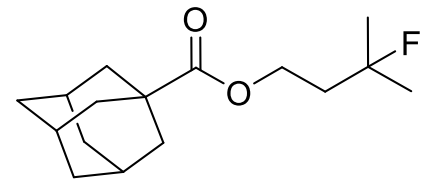

$2 p$

${ }^{19} \mathrm{~F} \mathrm{NMR,} 470 \mathrm{MHz}, \mathrm{CDCl}_{3}$
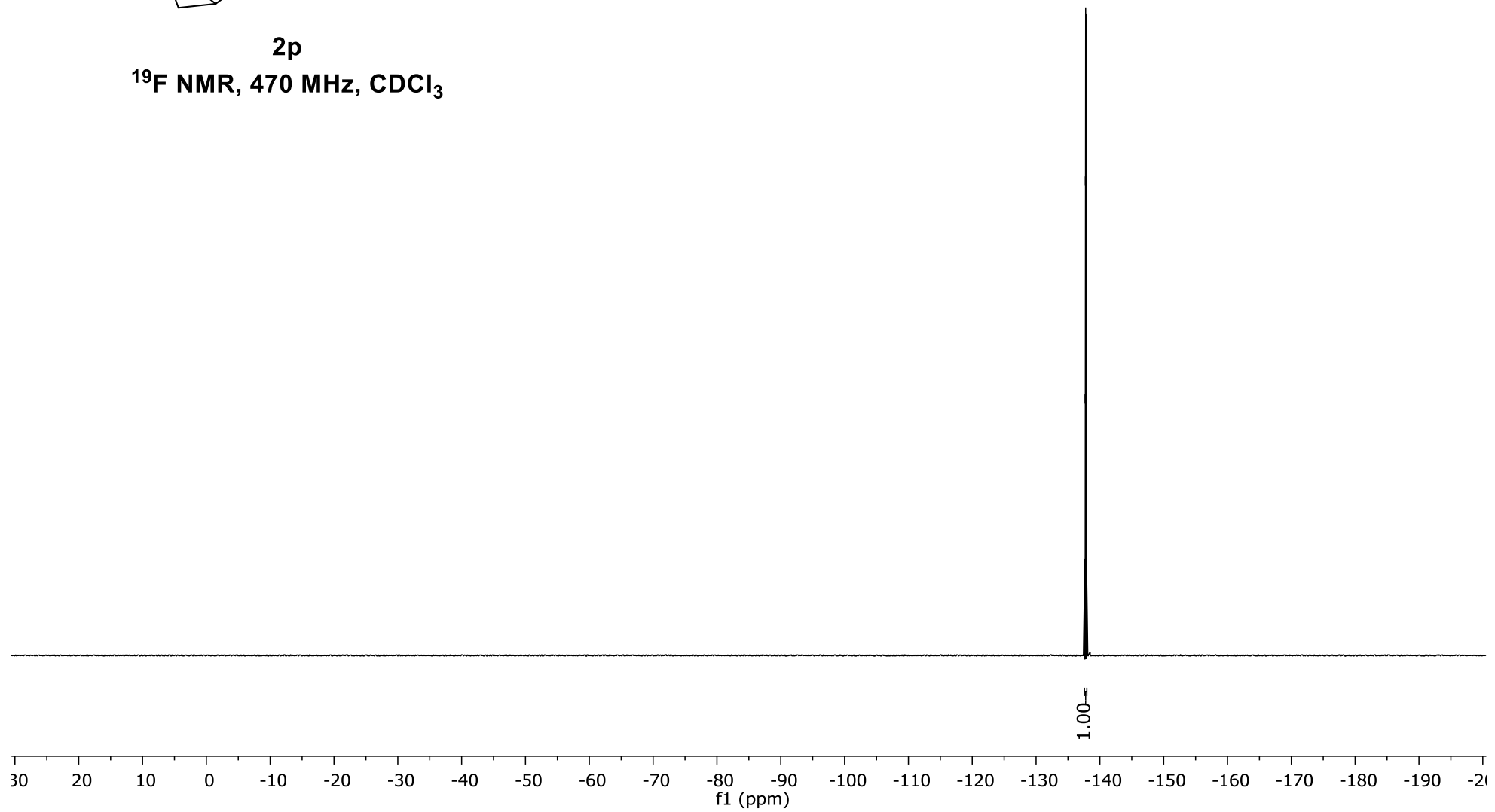


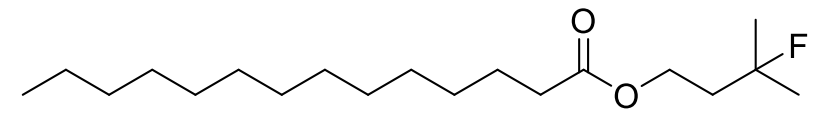

$2 q$

${ }^{1} \mathrm{H} \mathrm{NMR}, 500 \mathrm{MHz}, \mathrm{CDCl}_{3}$

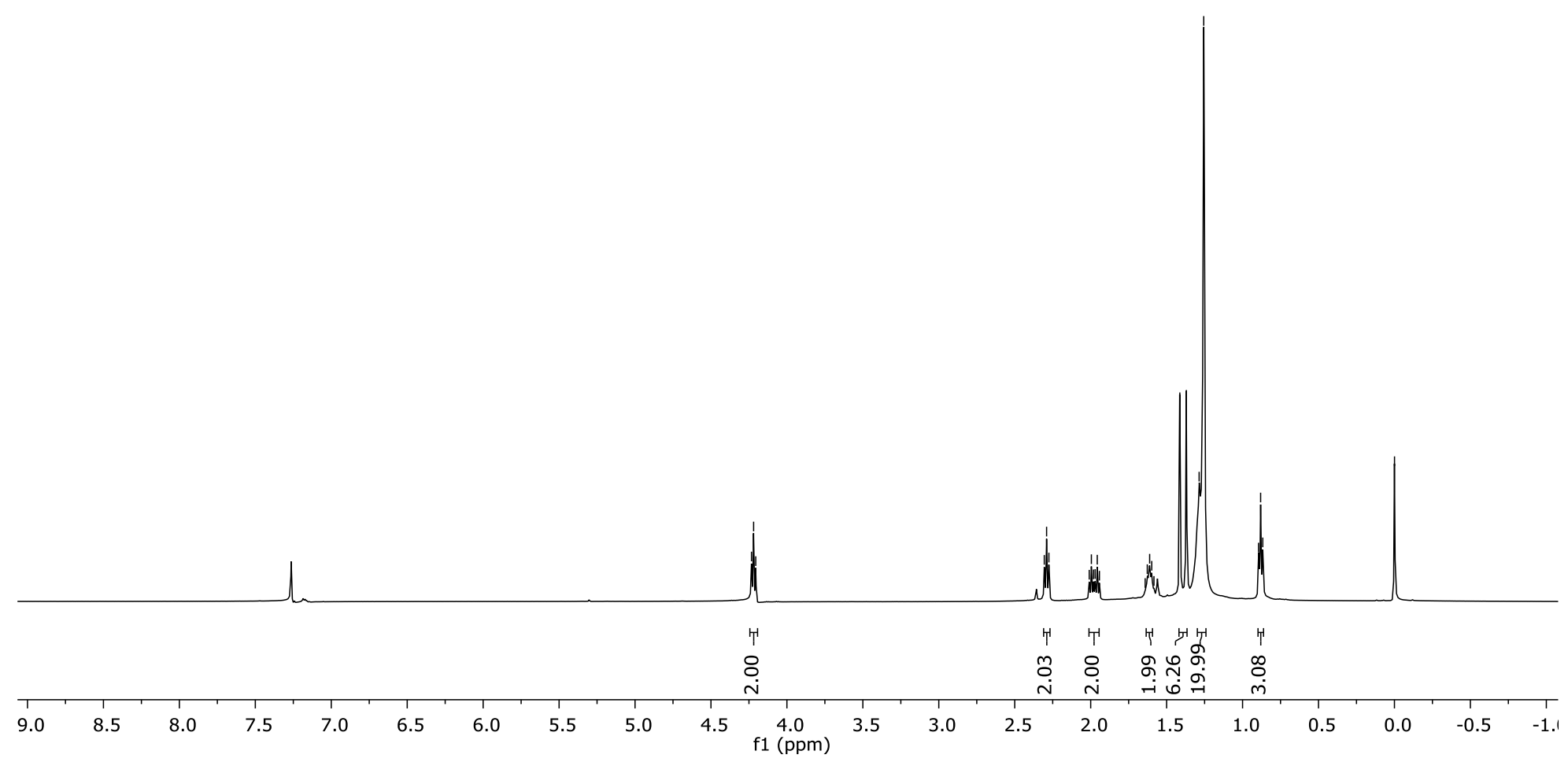




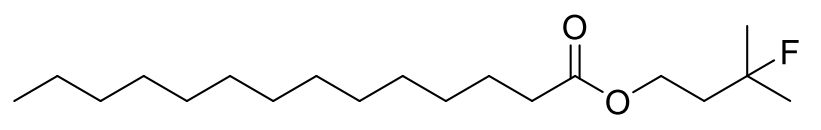

$2 q$

${ }^{13} \mathrm{C}$ NMR, $126 \mathrm{MHz}^{\mathrm{CDCl}}{ }_{3}$

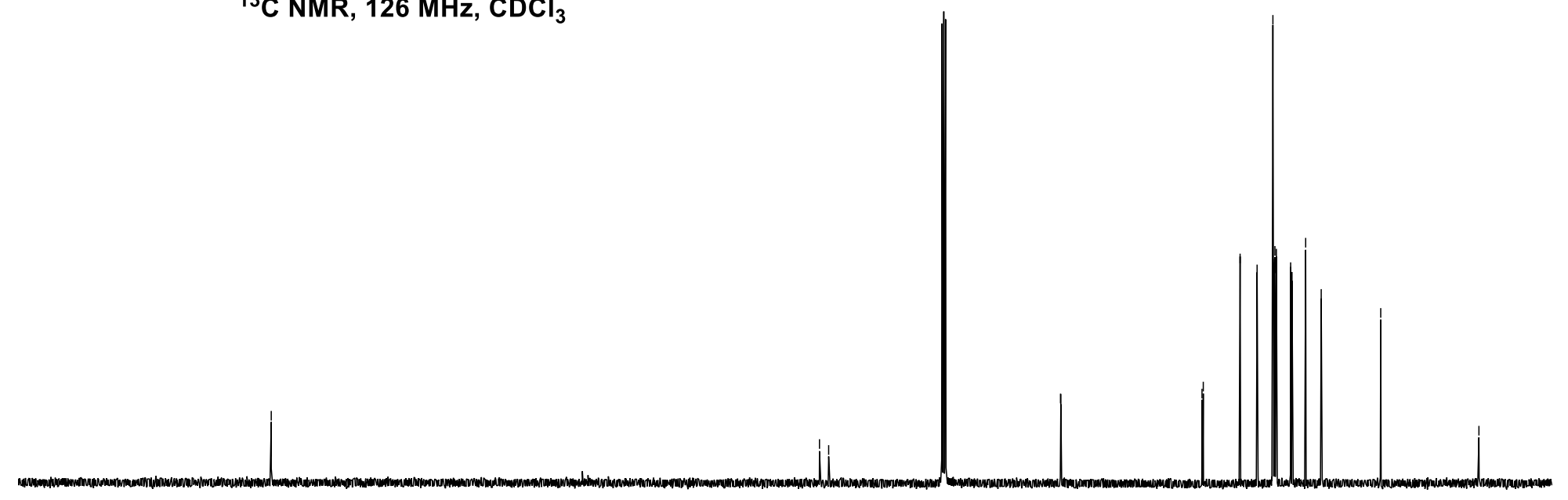

$10 \quad 200 \quad 190$ 


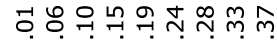

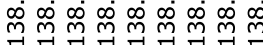

in

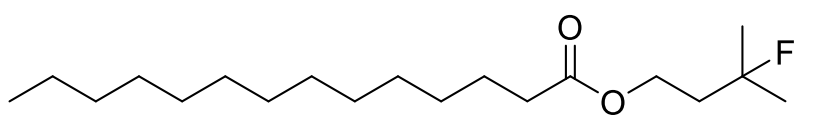

2q

${ }^{19} \mathrm{~F} \mathrm{NMR,}, 470 \mathrm{MHz}, \mathrm{CDCl}_{3}$

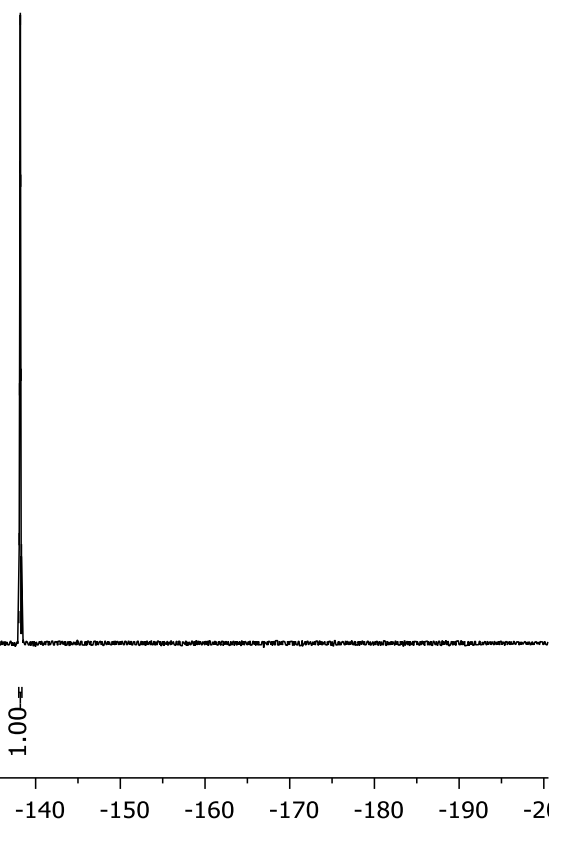

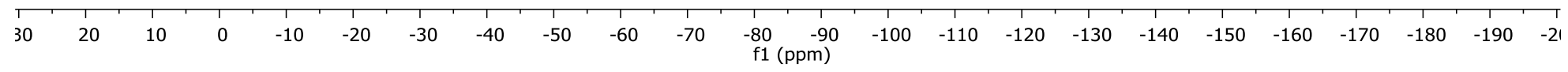




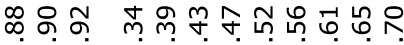

퍼

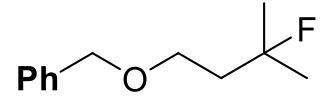

$2 \mathrm{r}$

${ }^{19} \mathrm{~F}$ NMR, $470 \mathrm{MHz}, \mathrm{CDCl}_{3}$

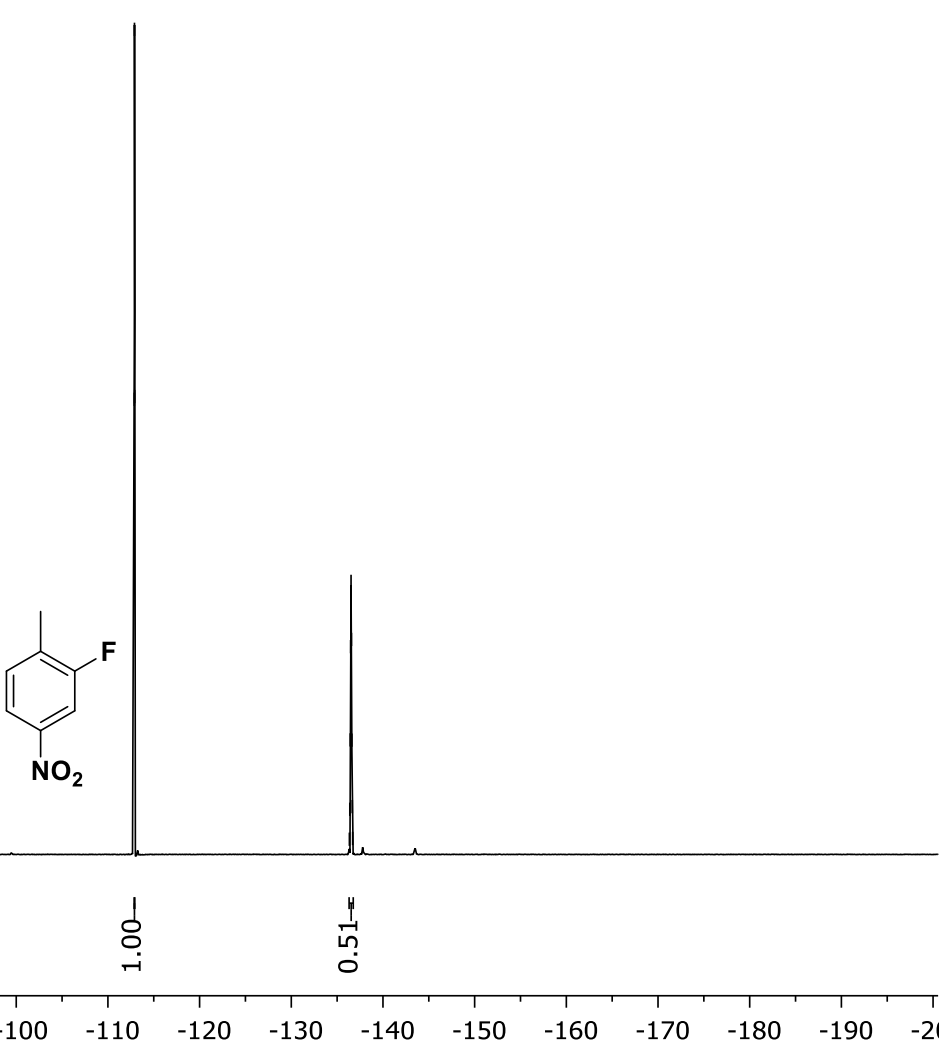




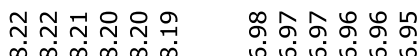

i.

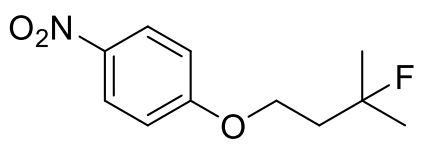

2s

${ }^{1} \mathrm{H}$ NMR, $500 \mathrm{MHz}, \mathrm{CDCl}_{3}$

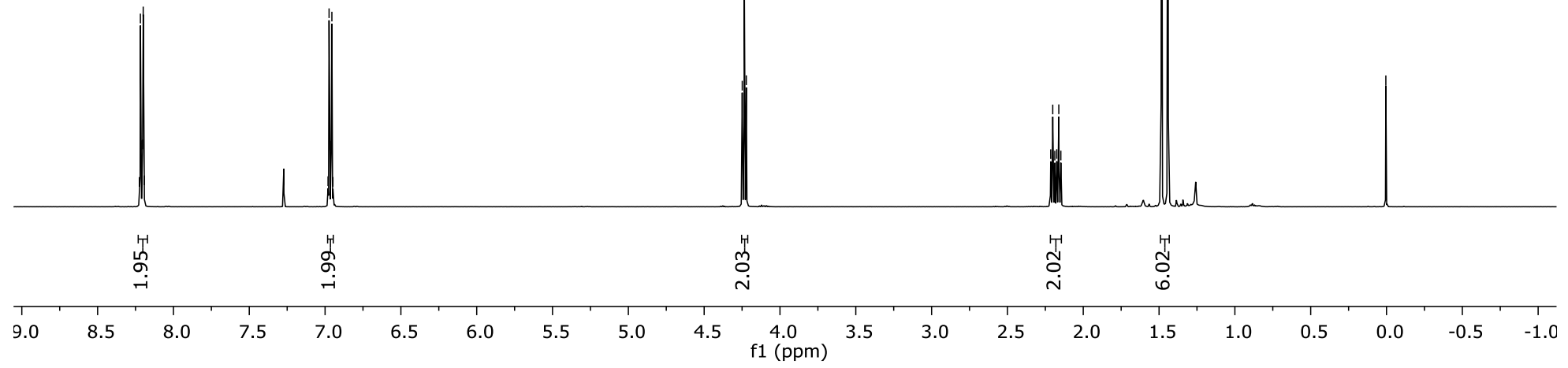




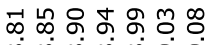

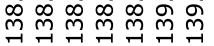

ris

$\mathrm{O}_{\mathrm{O}}^{\mathrm{O}}$

2s

${ }^{19} \mathrm{~F}$ NMR, $470 \mathrm{MHz}, \mathrm{CDCl}_{3}$

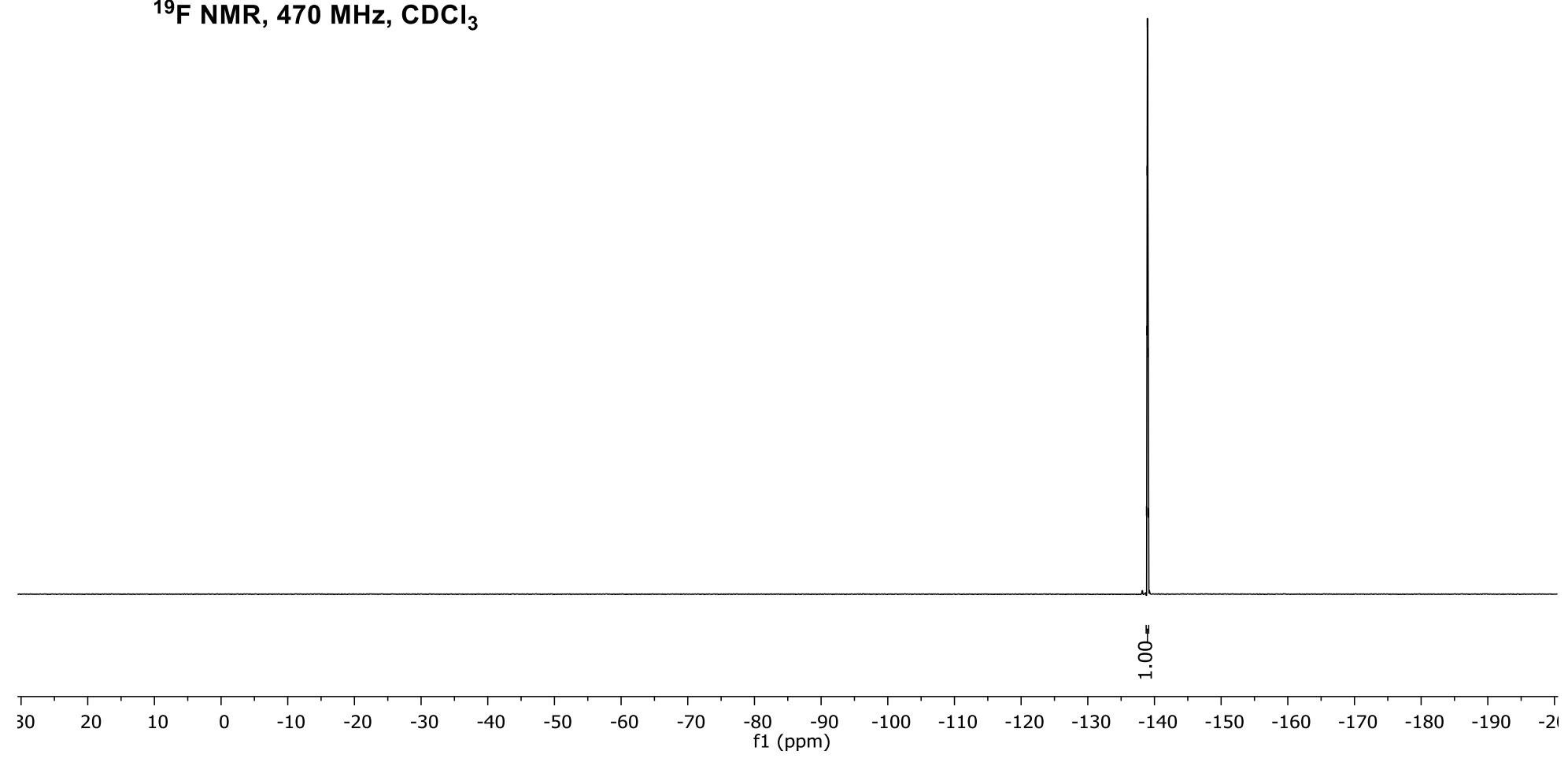




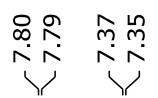

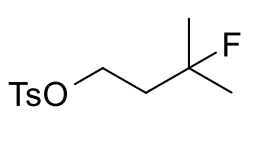

$2 t$

${ }^{1} \mathrm{H}$ NMR, $500 \mathrm{MHz}, \mathrm{CDCl}_{3}$

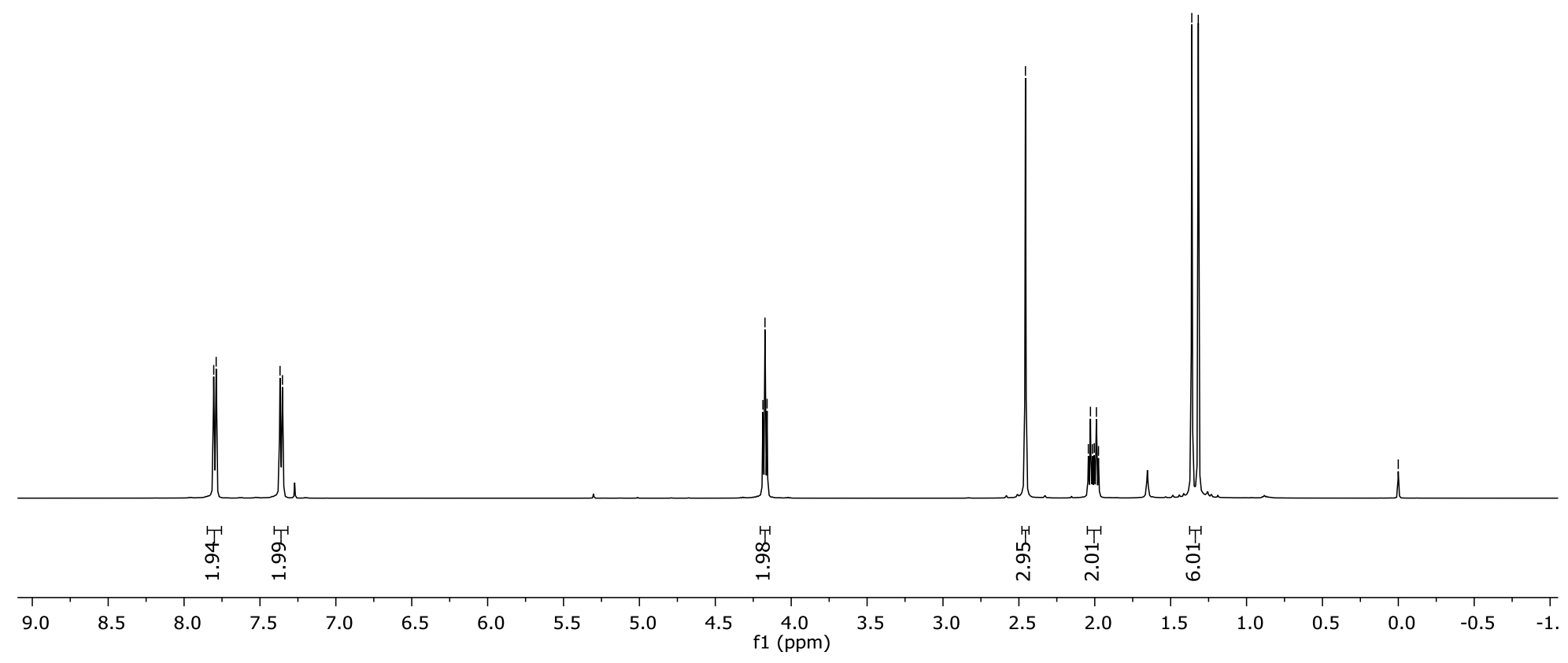




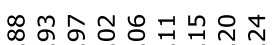

舫

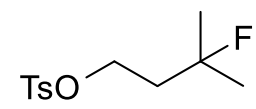

$2 t$

${ }^{19} \mathrm{~F}$ NMR, $470 \mathrm{MHz}, \mathrm{CDCl}_{3}$

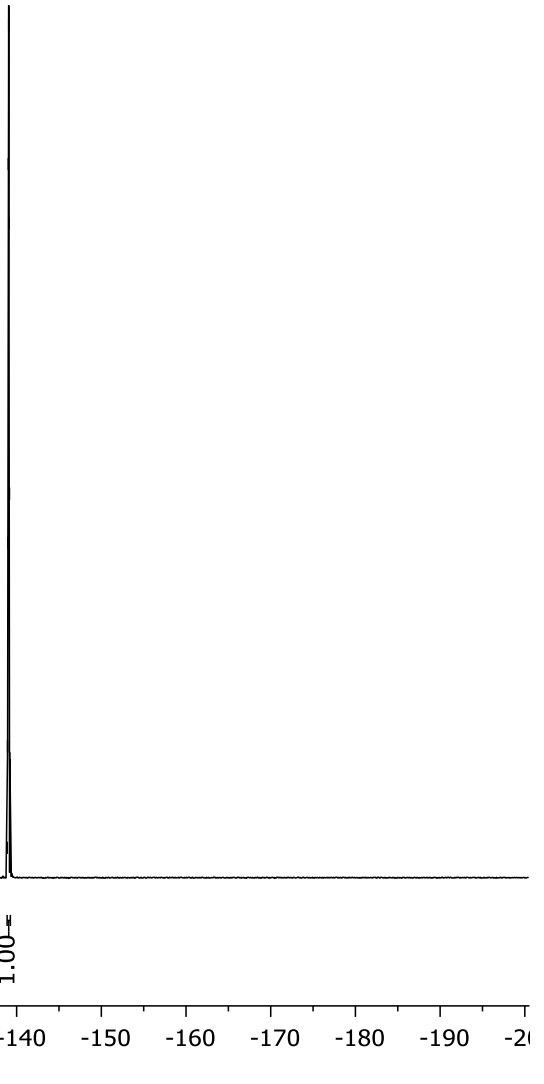

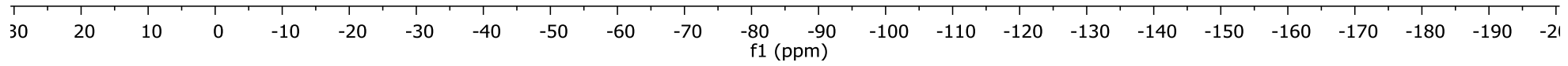




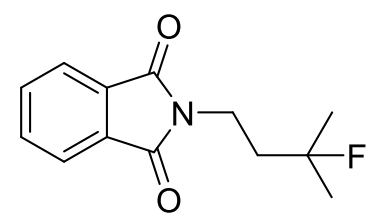

2u

${ }^{1} \mathrm{H}$ NMR, $500 \mathrm{MHz}, \mathrm{CDCl}_{3}$

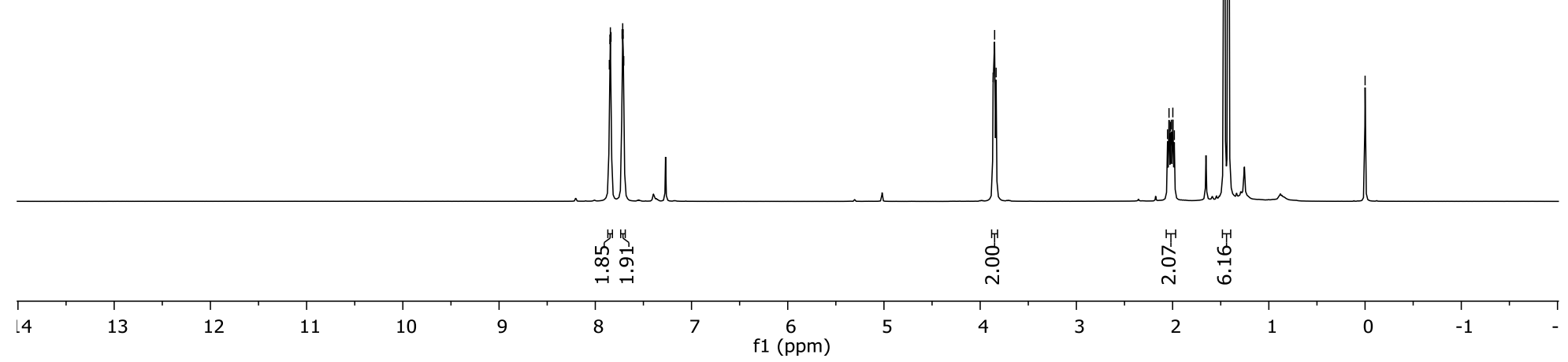




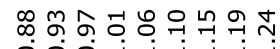

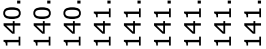

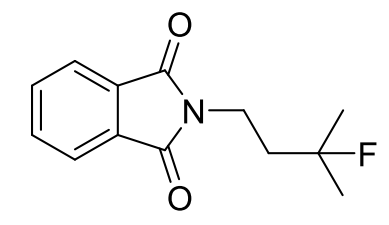

$2 \mathrm{u}$

${ }^{19} \mathrm{~F}$ NMR, $470 \mathrm{MHz}, \mathrm{CDCl}_{3}$

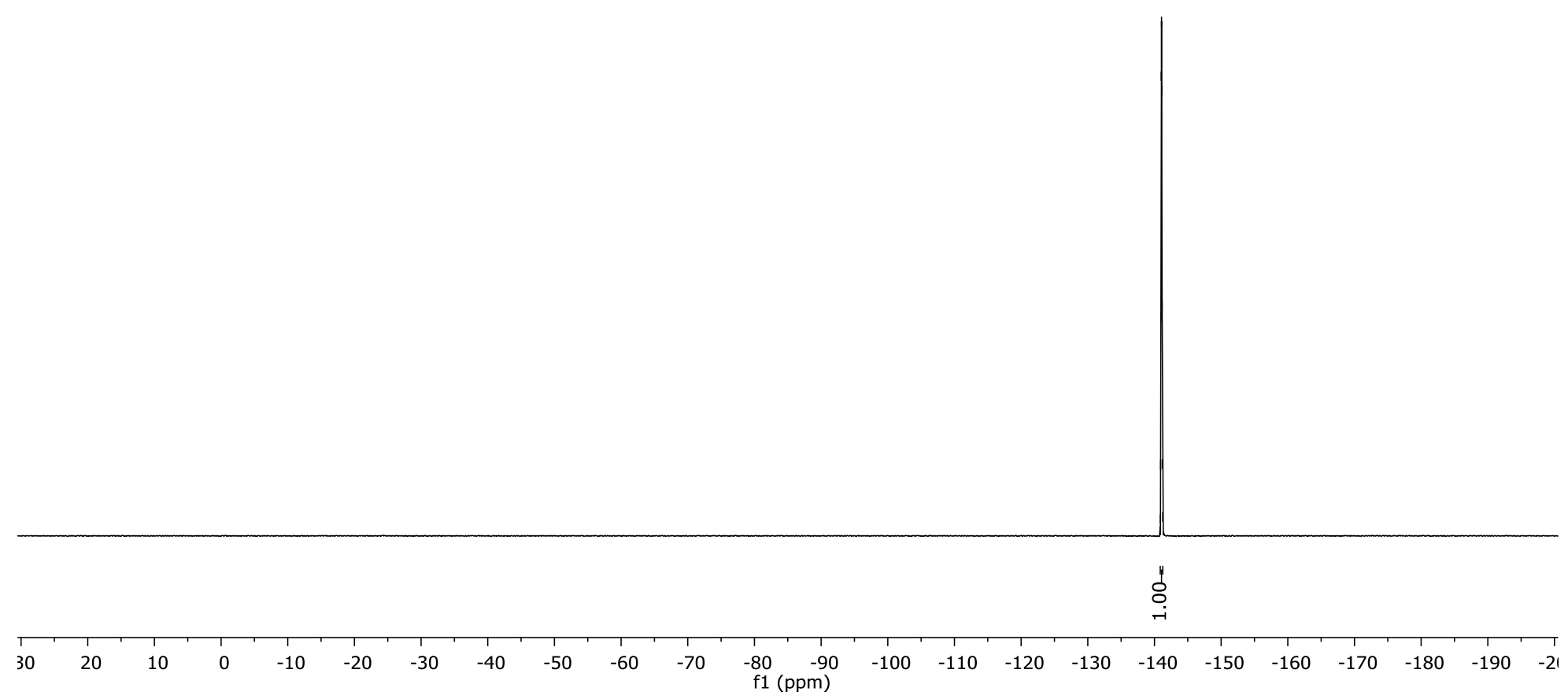




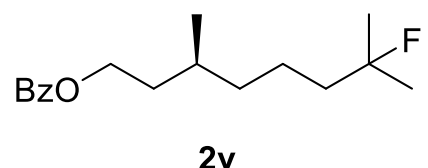

${ }^{1} \mathrm{H} \mathrm{NMR,}, 500 \mathrm{MHz}, \mathrm{CDCl}_{3}$

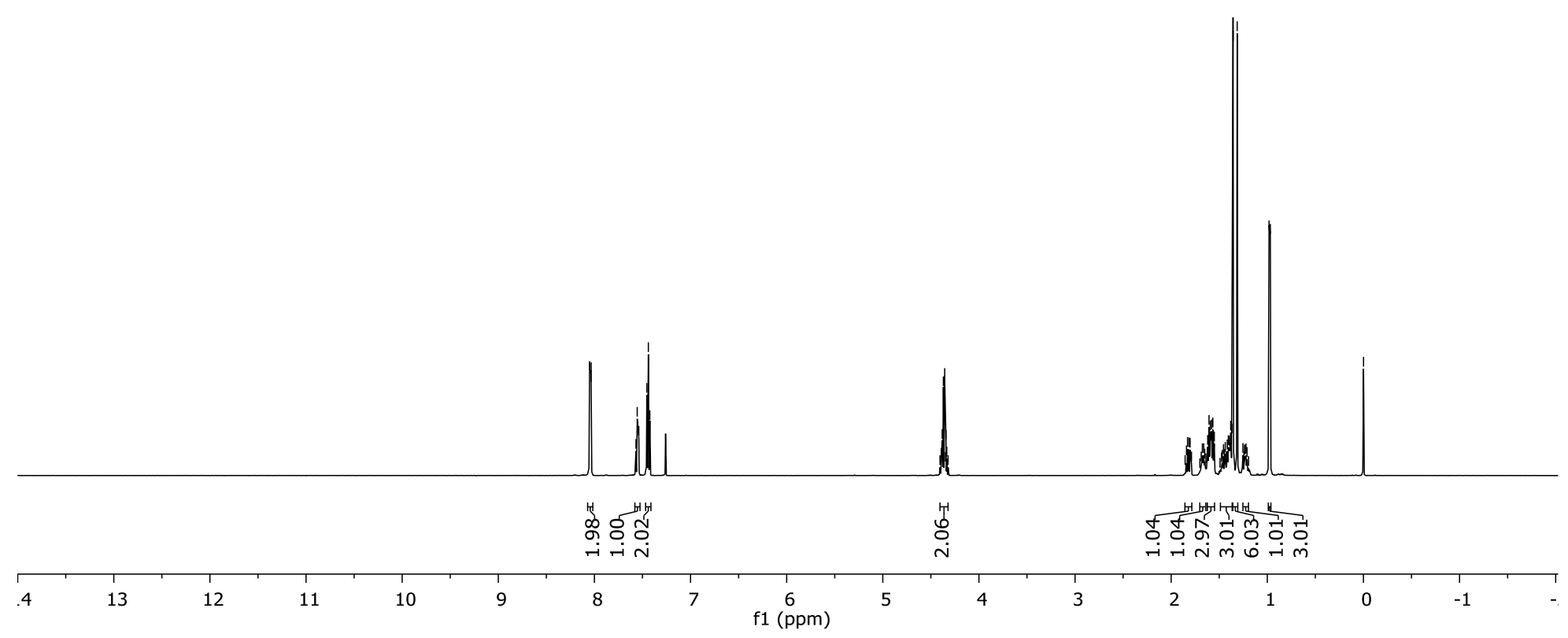




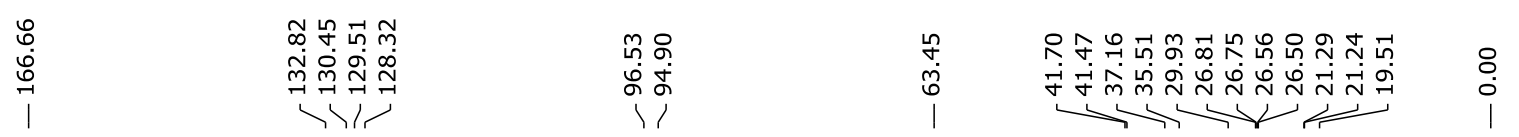

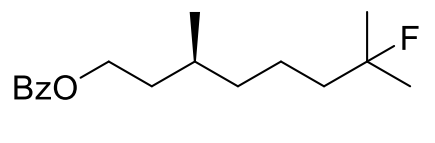

${ }^{13} \mathrm{C}$ NMR, $101 \mathrm{MHz}, \mathrm{CDCl}_{3}$

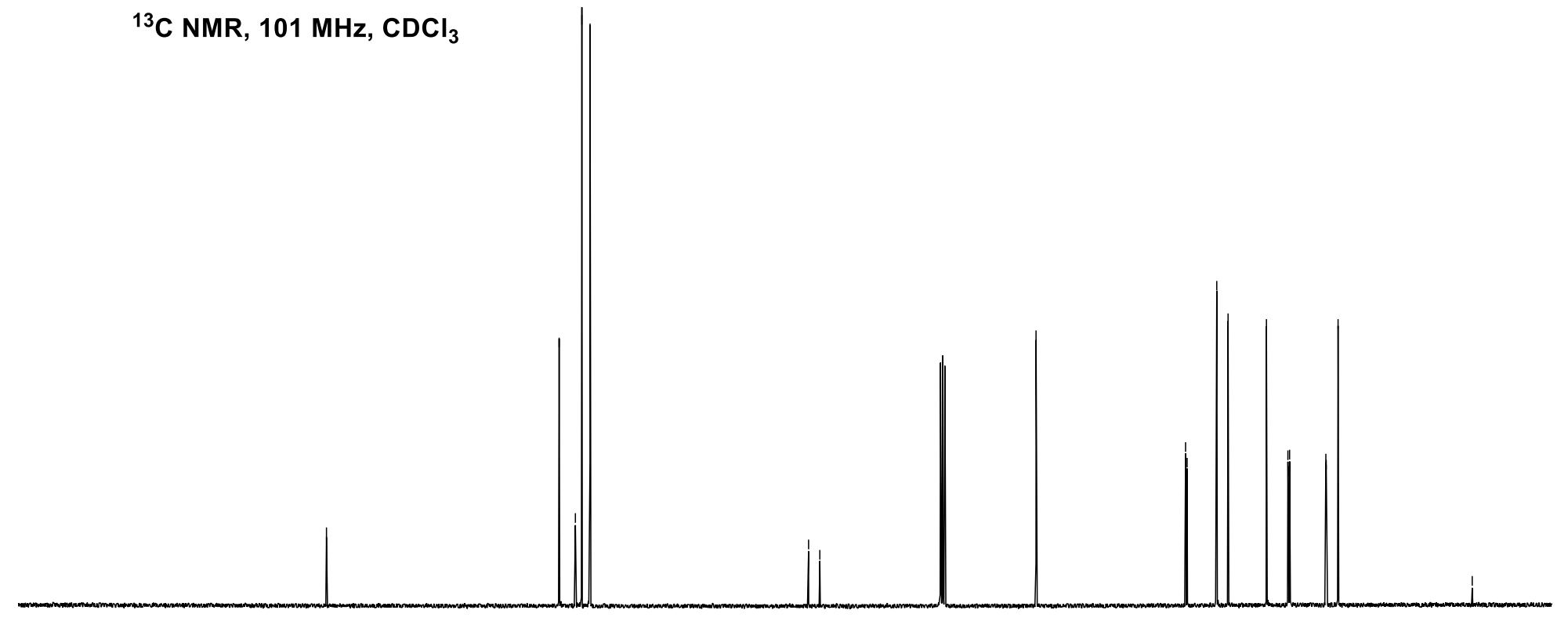


mై

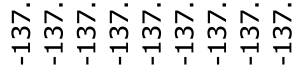

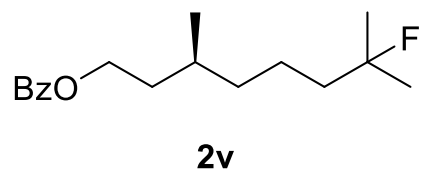

${ }^{19} \mathrm{~F}$ NMR, $470 \mathrm{MHz}, \mathrm{CDCl}_{3}$

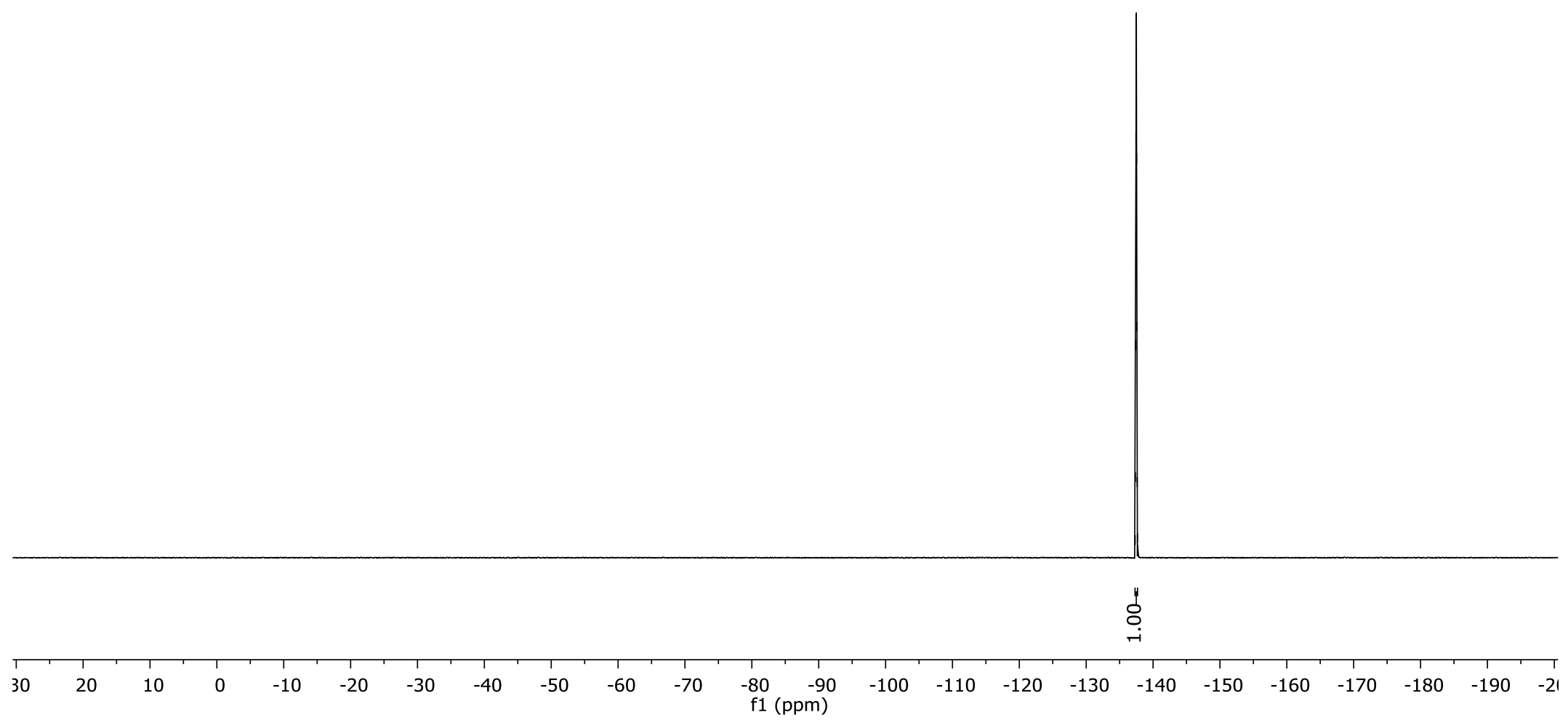




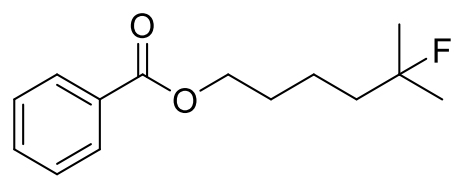

2w

${ }^{1} \mathrm{H}$ NMR, $400 \mathrm{MHz}, \mathrm{CDCl}_{3}$

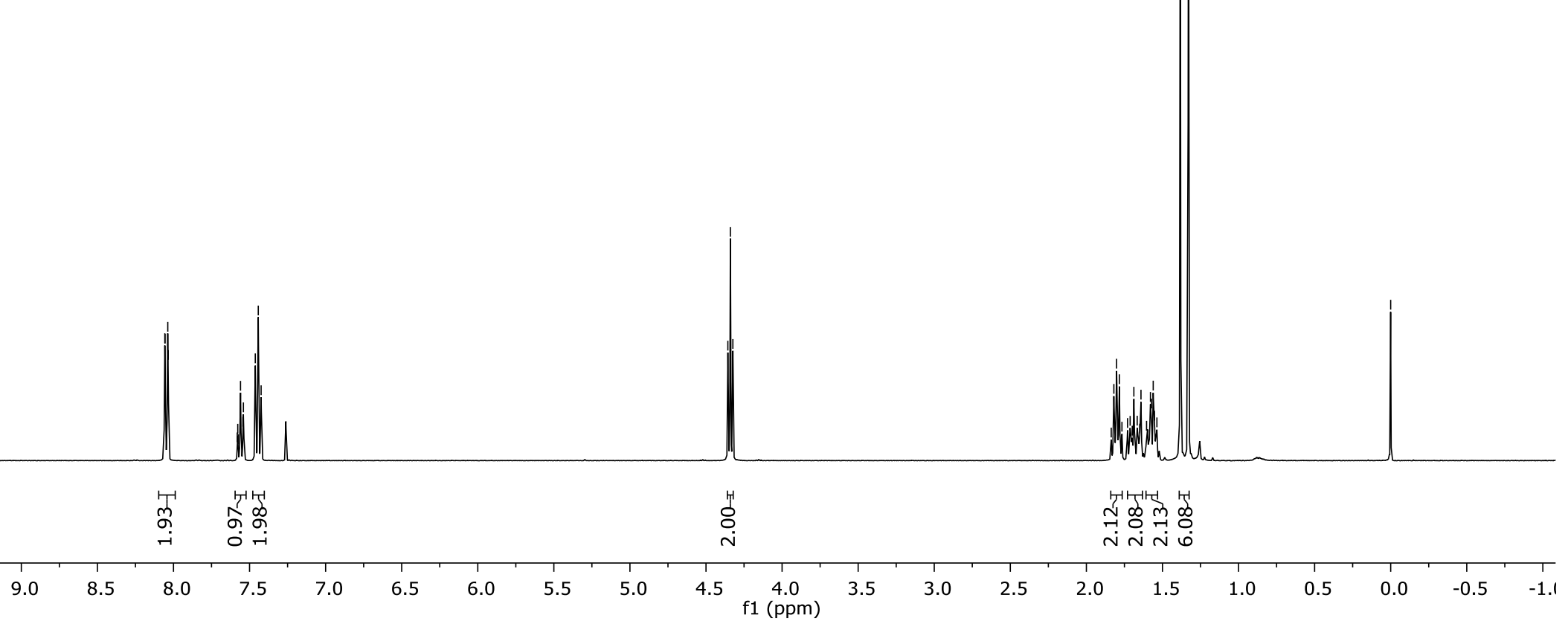




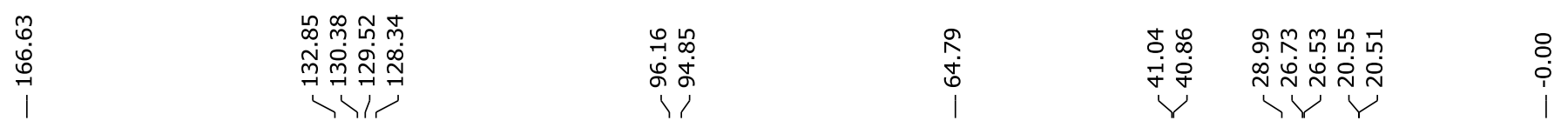

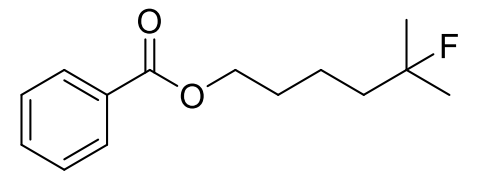

2w

${ }^{13} \mathrm{C} \mathrm{NMR,}, 126 \mathrm{MHz}, \mathrm{CDCl}_{3}$ 


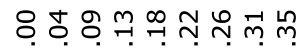

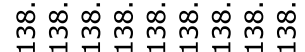

资

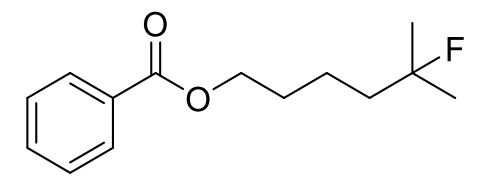

$2 w$

${ }^{19} \mathrm{~F} \mathrm{NMR}, 470 \mathrm{MHz}^{\mathrm{CDCl}}{ }_{3}$

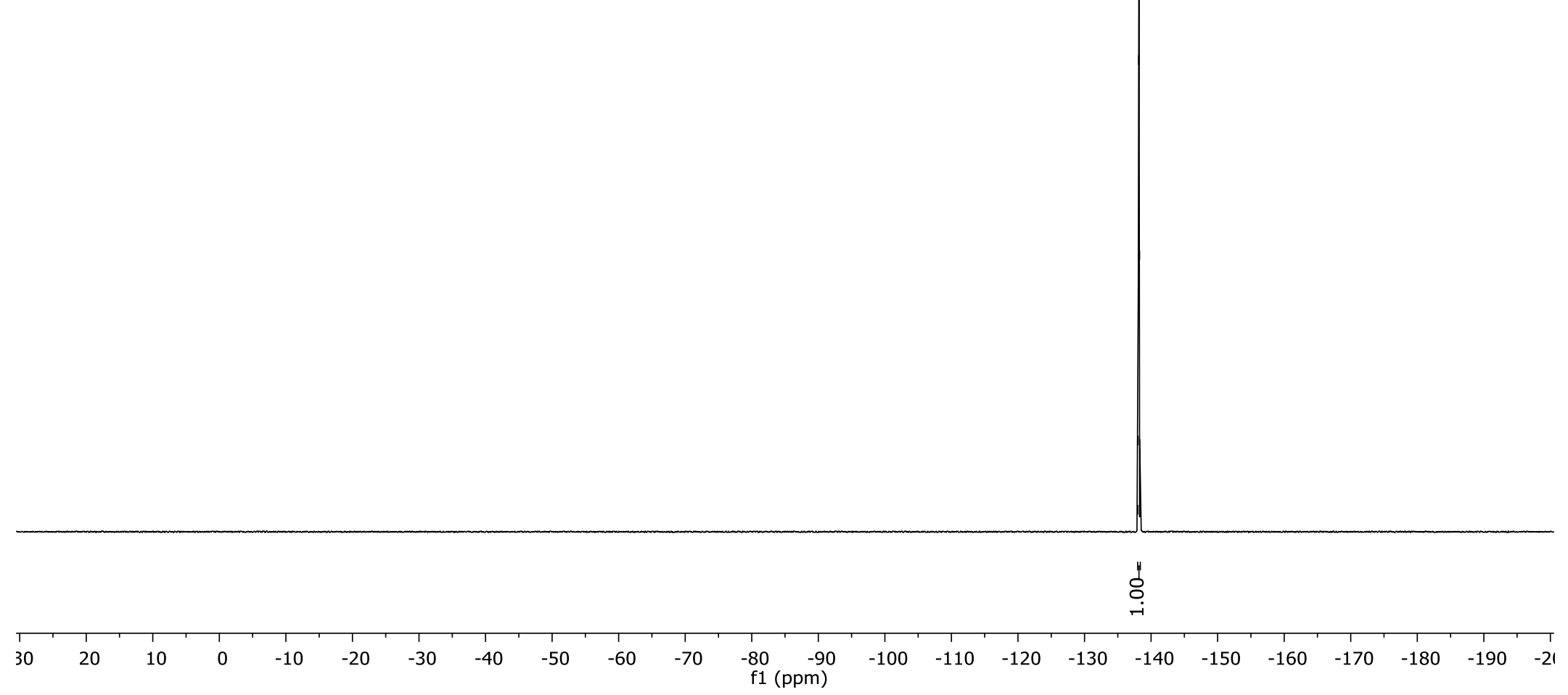




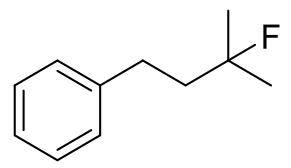

2x

${ }^{1} \mathrm{H}$ NMR, $400 \mathrm{MHz}, \mathrm{CDCl}_{3}$

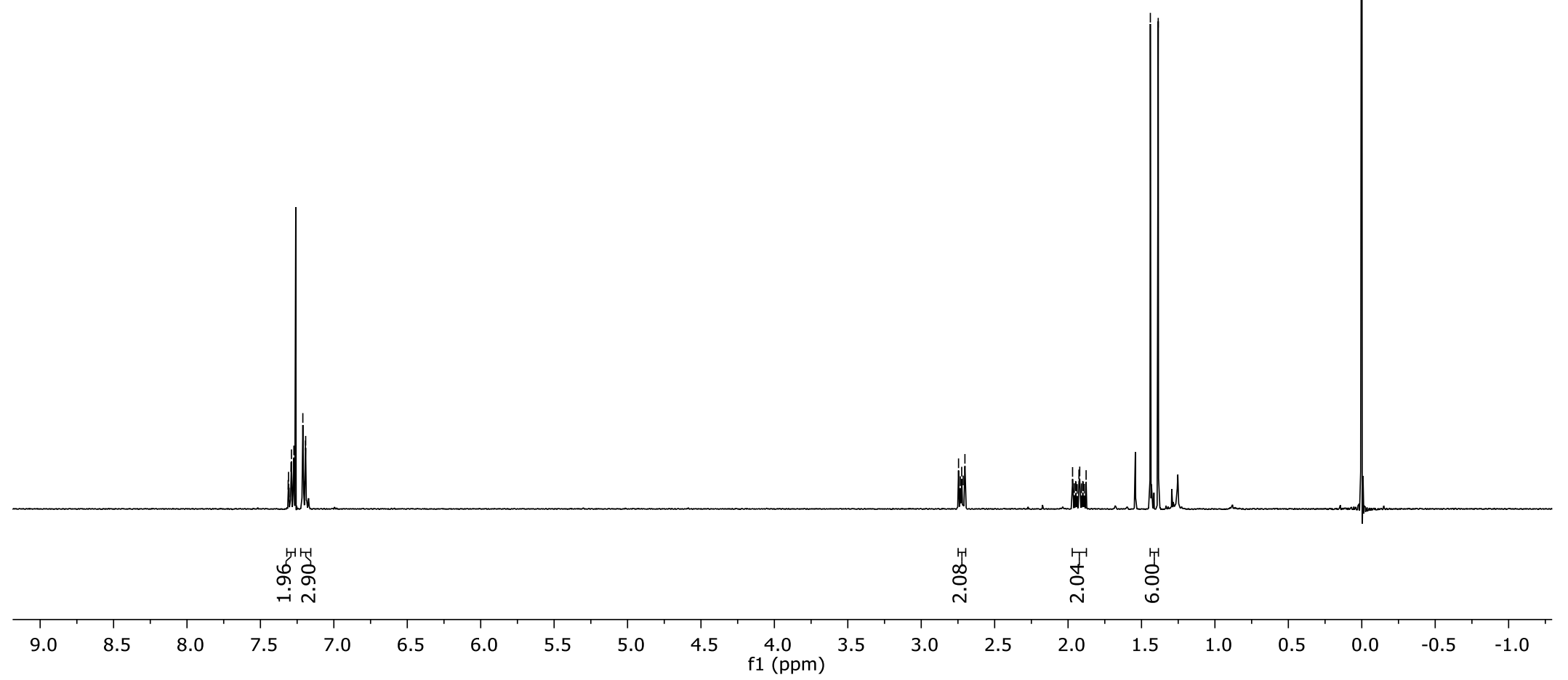




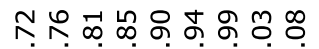

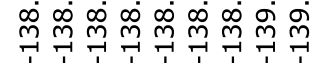

1

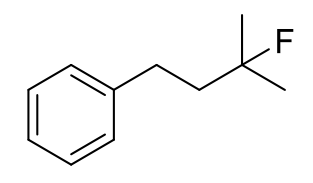

$2 \mathrm{x}$

${ }^{19} \mathrm{~F} \mathrm{NMR}, 470 \mathrm{MHz}, \mathrm{CDCl}_{3}$

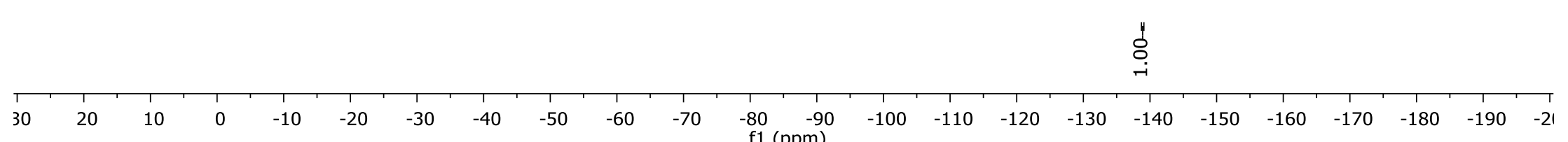




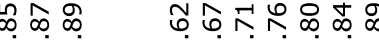

Ni

17

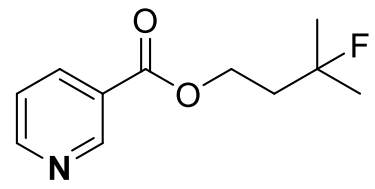

$2 y$

${ }^{19} \mathrm{~F} \mathrm{NMR}, 470 \mathrm{MHz}, \mathrm{CDCl}_{3}$

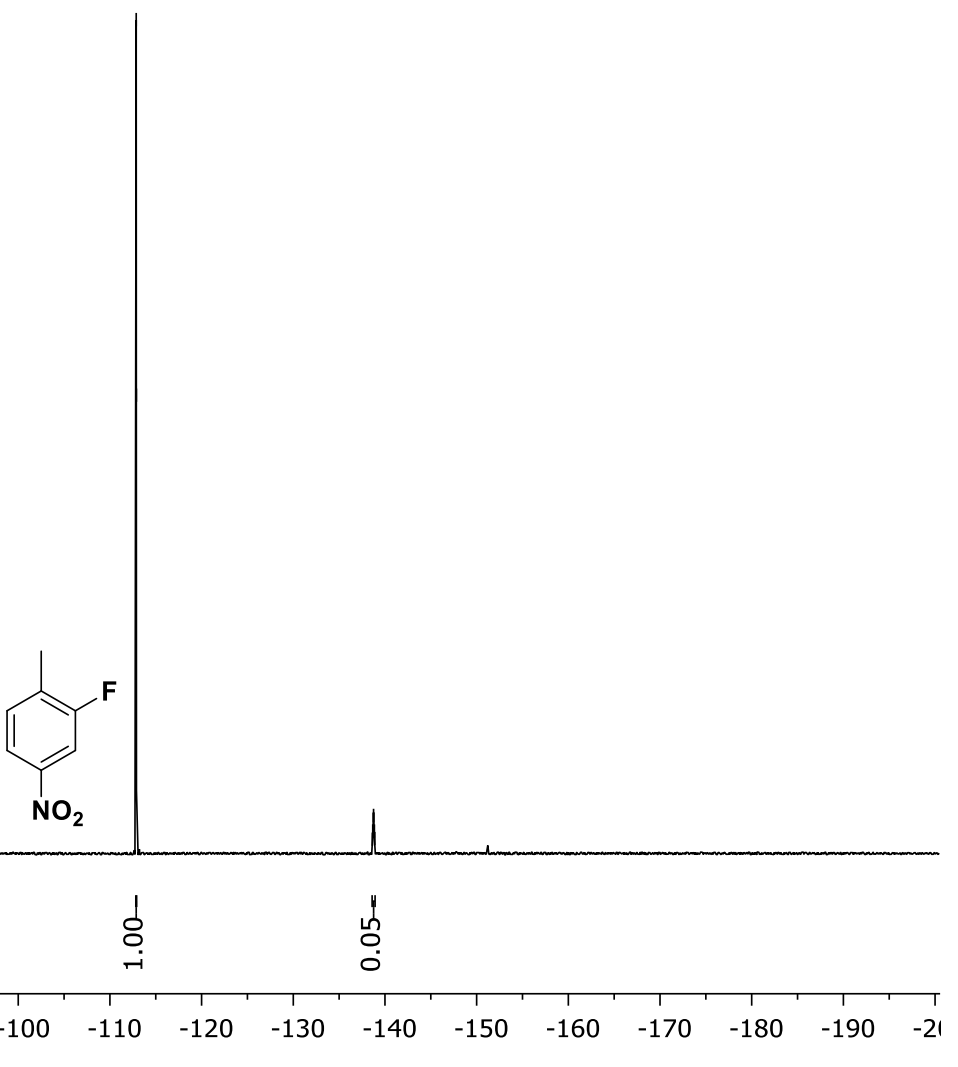




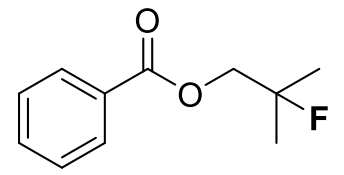

$2 z$

${ }^{19} \mathrm{~F}$ NMR, $470 \mathrm{MHz}, \mathrm{CDCl}_{3}$

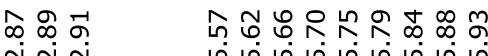

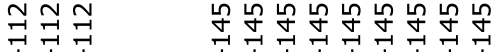

i'

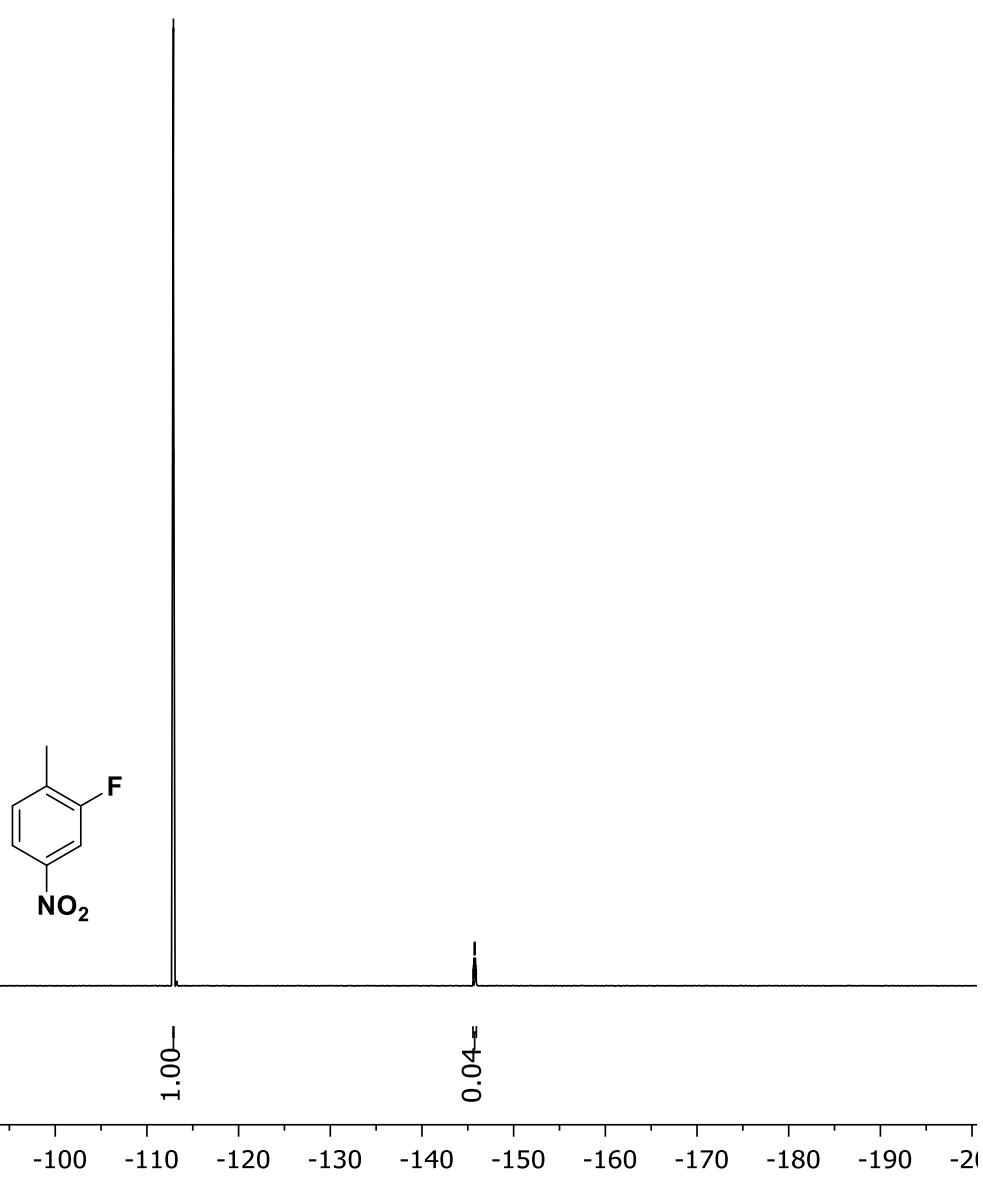

